\title{
SPECTRAL DEFORMATIONS OF ONE-DIMENSIONAL SCHRÖDINGER OPERATORS
}

\author{
By
}

F. GESZTESY, B. SIMON* AND G. TESCHL

\begin{abstract}
We provide a complete spectral characterization of a new method of constructing isospectral (in fact, unitary) deformations of general Schrödinger operators $H=-d^{2} / d x^{2}+V$ in $L^{2}(\mathbb{R})$. Our technique is connected to Dirichlet data, that is, the spectrum of the operator $H^{D}$ on $L^{2}\left(\left(-\infty, x_{0}\right)\right) \oplus L^{2}\left(\left(x_{0}, \infty\right)\right)$ with a Dirichlet boundary condition at $x_{0}$. The transformation moves a single eigenvalue of $H^{D}$ and perhaps flips which side of $x_{0}$ the eigenvalue lives. On the remainder of the spectrum, the transformation is realized by a unitary operator. For cases such as $V(x) \rightarrow \infty$ as $|x| \rightarrow \infty$, where $V$ is uniquely determined by the spectrum of $H$ and the Dirichlet data, our result implies that the specific Dirichlet data allowed are determined only by the asymptotics as $E \rightarrow \infty$.
\end{abstract}

\section{§1. Introduction}

Spectral deformations of Schrödinger operators in $L^{2}(\mathbb{R})$, isospectral and certain classes of non-isospectral ones, have attracted a lot of interest over the past three decades due to their prominent role in connection with a variety of topics, including the Korteweg-de Vries (KdV) hierarchy, inverse spectral problems, supersymmetric quantum mechanical models, level comparison theorems, etc. In fact, the construction of $N$-soliton solutions of the $\mathrm{KdV}$ hierarchy (and more generally, the construction of solitons relative to reflectionless backgrounds) is a typical example of a non-isospectral deformation of $H=-d^{2} / d x^{2}$ in $L^{2}(\mathbb{R})$ since the resulting deformation $\tilde{H}=-d^{2} / d x^{2}+\tilde{V}$ acquires an additional point spectrum $\left\{\lambda_{1}, \ldots, \lambda_{N}\right\} \subset(-\infty, 0)$ such that

$$
\sigma(\tilde{H})=\sigma(H) \cup\left\{\lambda_{1}, \ldots, \lambda_{N}\right\}
$$

$(\sigma(\cdot)$ abbreviating the spectrum). On the other hand, the solution of the inverse periodic problem and the corresponding solution of the algebro-geometric quasiperiodic finite-gap inverse problem for the $\mathrm{KdV}$ hierarchy (and certain almostperiodic limiting situations thereof) are intimately connected with isospectral (in fact, unitary) deformations of a given base (background) operator $H=-d^{2} / d x^{2}+V$. Although not a complete bibliography on applications of spectral deformations in

* This material is based upon work supported by the National Science Foundation under Grant No. DMS-9401491. The Government has certain rights in this material. 
mathematical physics, the interested reader may consult [1], [2], [3], [19], [31], [47], and the references cited therein.

Our main motivation in writing this paper descends from our interest in inverse spectral problems. As pointed out later (see Remarks 4.5, 4.7, and 4.8), spectral deformation methods can provide crucial insights into the isospectral class of a given base potential $V$, and in some cases can even determine the whole class $\operatorname{Iso}(V)=\left\{\tilde{V} \in L_{\text {loc }}^{1}(\mathbb{R}) \mid \sigma\left(-d^{2} / d x^{2}+\tilde{V}\right)=\sigma\left(-d^{2} / d x^{2}+V\right)\right\}$ of such potentials. A particularly "annoying" open problem in inverse spectral theory concerns the characterization of the isospectral class of potentials $V$ with purely discrete spectra (e.g., the harmonic oscillator $V(x)=x^{2}$ ).

In [24], we proposed a way to label the isospectral operators for such a situation with Dirichlet data. Fix $x_{0}$ and let $H^{D}$ be the operator in $L^{2}\left(\left(-\infty, x_{0}\right)\right) \oplus L^{2}\left(\left(x_{0}, \infty\right)\right)$ with Dirichlet boundary condition at $x_{0} . H^{D}=H_{-}^{D} \oplus H_{+}^{D}$. The Dirichlet data are the pairs $(\mu, \sigma)$ with $\mu \in \mathbb{R}, \sigma \in\{+,-\}$ of eigenvalues of $H^{D}$ and a label of whether they are eigenvalues of $H_{-}^{D}$ or $H_{+}^{D}$. We showed in [23], Theorem 3.6, that for any Dirichlet data, there is at most one $V$ in the isospectral class of a given $-d^{2} / d x^{2}+V_{0}$ with discrete spectrum so that $V$ has the given Dirichlet data (in the degenerate case, where any eigenvalues of $H^{D}$ and $H$ coalesce, one must include an additional parameter in the Dirichlet data for each coincidence of eigenvalues, see Remarks 4.9 and 4.10). That is, the map from $V$ to Dirichlet data is one-one when defined on the isospectral set of potentials. The issue is determining the range of this map.

While this paper does not solve the inverse discrete spectral problem, it will make one important contribution. As a result of our principal Theorem 4.4, we obtain the fact that for any potential $V$, any finite number of deformations of Dirichlet data (i.e., Dirichlet eigenvalues together with their left/right half-line distribution, see (2.7)) in spectral gaps of $V$ produce isospectral deformations $\tilde{V} \in \operatorname{Iso}(V)$ of $V$. In particular, there are no further constraints on these Dirichlet data (except, of course, these deformations are required to be finite in number and to stay within the spectral gaps in question). Applied to the inverse discrete spectral problem, this means that any constraints enforced on Dirichlet data can only be asymptotic in nature, that is, can only come from their "tail end" at infinity. That such asymptotic constraints necessarily exist is a consequence of a recently proved general trace formula for $V(x)$ [24] (see Remark 4.8). The precise nature of these constraints, however, is unknown to date.

Mathematically, the techniques involved to produce isospectral $\tilde{V}$ or classes of non-isospectral ones where eigenvalues are added or removed, but the remaining spectral characteristics stay identically to those of the base potential $V$, can be traced back to commutation methods. These commutation methods in turn are intimately connected with factorizations of the Schrödinger differential expression $-d^{2} / d x^{2}+V(x)$ into products of first-order differential expressions. More precisely, 
one seeks a factorization of the type

$$
\begin{gathered}
-\frac{d^{2}}{d x^{2}}+V(x)=\alpha(\lambda) \alpha(\lambda)^{+}+\lambda \\
\alpha(\lambda)=\frac{d}{d x}+\phi(\lambda, x), \quad \alpha(\lambda)^{+}=-\frac{d}{d x}+\phi(\lambda, x)
\end{gathered}
$$

for some appropriate $\lambda \in \mathbb{R}$. A subsequent commutation of the factors $\alpha(\lambda)$ and $\alpha(\lambda)^{+}$, introducing the differential expression

$$
-\frac{d^{2}}{d x^{2}}+\tilde{V}(\lambda, x)=\alpha(\lambda)^{+} \alpha(\lambda)+\lambda
$$

then yields associated isospectral or special classes of non-isospectral deformations $\tilde{V}(\lambda, x)$ of $V(x)$ depending on the choice of $\phi(\lambda, x)$ in $\alpha(\lambda), \alpha(\lambda)^{+}$. In the following, we briefly outline three different instances of commutation techniques that occur in the literature.

We start with the single commutation or Crum-Darboux method (going back at least to Jacobi). In this method, $H=-d^{2} / d x^{2}+V$ is assumed to be bounded from below, inf $\sigma(H)>-\infty$, and $\lambda \in \mathbb{R}$ is chosen according to $\lambda<\inf \sigma(H)$. One sets $\phi(\lambda, x)=\psi_{\nu}^{\prime}(\lambda, x) / \psi_{\nu}(\lambda, x)$, where $\psi_{\nu}$ satisfies $\psi^{\prime \prime}=(V-\lambda) \psi$ (cf. (A.7)). Depending on the choice of $\psi_{\nu}(\lambda, x)$, the aforementioned commutation procedure yields a spectral deformation $\tilde{H}_{\nu}(\lambda)$ of $H$,

$$
\begin{gathered}
\tilde{H}_{\nu}(\lambda)=-\frac{d^{2}}{d x^{2}}+\tilde{V}_{\nu}(\lambda, x), \quad \tilde{V}_{\nu}(\lambda, x)=V(x)-2\left\{\ln \left[\psi_{\nu}(\lambda, x)\right]\right\}^{\prime \prime}, \\
x \in \mathbb{R}, \quad \lambda<\inf \sigma(H),
\end{gathered}
$$

which is either isospectral to $H$ or acquires the additional eigenvalue $\lambda$ below the spectrum of $H$, that is,

$$
\text { either } \sigma\left(\tilde{H}_{\nu}(\lambda)\right)=\sigma(H) \text { or } \quad \sigma\left(\tilde{H}_{\nu}(\lambda)\right)=\sigma(H) \cup\{\lambda\}
$$

Moreover, it can be proved that the remaining spectral characteristics of $H$ remain preserved in the sense that $\tilde{H}_{\nu}(\lambda)$ and $H$, restricted to the orthogonal complement of the eigenspace associated with $\lambda$, are unitarily equivalent.

A summary of this technique, as well as pertinent references to its extraordinary history and to more recent applications of it, will be given in Appendix A.

The fact that $\lambda$ is required to lie below the spectrum of $H$ is clearly a severe limitation. One possibility to avoid this restriction is provided by the following second technique, the double commutation method. 
Formally, this method can be obtained from two successive single commutations at a point $\lambda \in \mathbb{R} \backslash \sigma_{\mathrm{ess}}(H)\left(\sigma_{\mathrm{ess}}(\cdot)\right.$ the essential spectrum), or equivalently, as the result of two single commutations at $\lambda^{\prime}$ and $\lambda^{\prime \prime}, \lambda^{\prime} \neq \lambda^{\prime \prime}, \lambda^{\prime}, \lambda^{\prime \prime} \in \mathbb{R} \backslash \sigma_{\text {ess }}(H)$ with a subsequent limiting procedure $\lambda^{\prime} \rightarrow \lambda$ and $\lambda^{\prime \prime} \rightarrow \lambda$. The final outcome can be sketched as follows. Pick $\gamma>0, \lambda \in \mathbb{R} \backslash \sigma_{\text {ess }}(H)$ and real-valued $\psi_{ \pm}(\lambda, \cdot) \in$ $L^{2}((R, \pm \infty)), R \in \mathbb{R}$ satisfying $\psi^{\prime \prime}(\lambda)=(V-\lambda) \psi(\lambda)$. The spectral deformation $\tilde{H}_{ \pm, \gamma}(\lambda)$ of $H$ is then given by

$$
\begin{aligned}
\tilde{H}_{ \pm, \gamma}(\lambda) & =-\frac{d^{2}}{d x^{2}}+\tilde{V}_{ \pm, \gamma}(\lambda, x), \\
\tilde{V}_{ \pm, \gamma}(\lambda, x) & =V(x)-2\left\{\ln \left[1 \mp \gamma \int_{ \pm \infty}^{x} d x^{\prime} \psi_{ \pm}\left(\lambda, x^{\prime}\right)^{2}\right]\right\}^{\prime \prime}, \quad \gamma>0, \lambda \in \mathbb{R} \backslash \sigma_{\mathrm{ess}}(H) .
\end{aligned}
$$

In this case, one can show that

$$
\sigma\left(\tilde{H}_{ \pm, \gamma}(\lambda)\right)=\sigma(H) \cup\{\lambda\}
$$

and again $\tilde{H}_{ \pm, \gamma}(\lambda)$ and $H$ are unitarily equivalent upon restriction onto the orthogonal complements of their eigenspaces corresponding to $\lambda$.

A summary of this method together with appropriate references to its history, as well as to recent applications of it, will be provided in Appendix B.

Finally, and most importantly in connection with the contents of this paper, we shall describe a third commutation method first introduced by Finkel, Isaacson, and Trubowitz [12] in 1987 in connection with an explicit realization of the isospectral torus of periodic potentials. This method was again used by Buys and Finkel [4] (see also Iwasaki [32]) in the context of periodic finite-gap potentials and by Pöschel and Trubowitz [48] and Ralston and Trubowitz [49] for various boundary value problems on compact intervals. As in the previous case, this method formally consists of two single commutations, but this time at different values of the spectral parameter. The principal contribution of this paper is a generalization of the work of Finkel, Isaacson, and Trubowitz to arbitrary (i.e,, not necessarily periodic) base potentials $V(x)$ and a complete spectral characterization of this commutation technique. As a result we obtain a powerful new tool in constructing sets of isospectral potentials for arbitrary base potentials.

We briefly sketch this approach. Suppose $\psi_{ \pm}(z, \cdot) \in L^{2}((R, \pm \infty)), z \in \mathbb{C} \backslash \sigma_{\text {ess }}(H)$, $R \in \mathbb{R}$ satisfy $\psi^{\prime \prime}(z)=(V-z) \psi(z)$, and pick a spectral gap $\left(E_{0}, E_{1}\right)$ of $H$ with $\mu, \tilde{\mu} \in\left(E_{0}, E_{1}\right)$. Define

$$
W_{(\tilde{\mu}, \tilde{\sigma})}(x)=(\tilde{\mu}-\mu)^{-1} W\left(\psi_{\sigma}(\mu), \psi_{-\tilde{\sigma}}(\tilde{\mu})\right)(x), \quad \sigma, \tilde{\sigma} \in\{-,+\}, x \in \mathbb{R}
$$


where $W(f, g)(x)=f(x) g^{\prime}(x)-f^{\prime}(x) g(x)$ denotes the Wronskian of $f$ and $g$ (taking limits if $\tilde{\mu}=\mu$ ). The spectral deformation $\tilde{H}_{(\tilde{\mu}, \tilde{\sigma})}$ of $H$ is then given by

$$
\tilde{H}_{(\tilde{\mu}, \tilde{\sigma})}=-\frac{d^{2}}{d x^{2}}+\tilde{V}_{(\tilde{\mu}, \tilde{\sigma})}, \quad \tilde{V}_{(\tilde{\mu}, \tilde{\sigma})}(x)=V(x)-2\left\{\ln \left[W_{(\tilde{\mu}, \tilde{\sigma})}(x)\right]\right\}^{\prime \prime}
$$

In order to define $\tilde{V}_{(\tilde{\mu}, \tilde{\sigma})}$, one needs, of course, to show that $W_{(\tilde{\mu}, \tilde{\sigma})}(x)$ is nonvanishing on $\mathbb{R}$. Indeed, the key to our extension of this method to the whole line is precisely our proof in [25] that this Wronskian is non-zero. This proof avoids the indirect argument of [12], [48], [49] that relies on compactness of the underlying interval. (Even if one is only interested in the compact interval case, our direct proof is simpler than their indirect argument.) In addition to allowing the extension to whole line problems in principle, this paper provides explicit calculations in the change of Weyl-Titchmarsh and spectral functions.

In our main result, Theorem 4.4 , we shall prove that

$$
\sigma\left(\tilde{H}_{(\tilde{\mu}, \tilde{\sigma})}\right)=\sigma(H)
$$

in fact, $\tilde{H}_{(\tilde{\mu}, \tilde{\sigma})}$ and $H$ will turn out to be unitarily equivalent. Moreover, if $(\mu, \sigma)$ is a Dirichlet datum for $H$ with respect to the reference point $x_{0}$, then all Dirichlet data for $\tilde{H}_{(\tilde{\mu}, \tilde{\sigma})}$ with respect to $x_{0}$ are identical to those of $H$, except that $(\mu, \sigma)$ is removed and $(\tilde{\mu}, \tilde{\sigma})$ is added instead. These results and a variety of extensions thereof constitute the principal new material in this paper. Because the spectral types of all operators in [12], [48], and [49] are explicitly known, the unitarity theorem is a trivial consequence of the determination of spectra. However, for general base potentials, the spectral types can be exotic so that the unitarity result is much stronger than a mere equality result of spectra. Our proof of the unitarity relies on the explicit formula of the changes in the spectral matrix.

Section 2 provides the background needed in the remainder of this paper. Section 3 treats Weyl-Titchmarsh $m$-functions and spectral functions associated with half-line Dirichlet operators. Section 4 contains our principal results on isospectral deformations and provides a complete spectral characterization of this deformation method. In particular, the Weyl-Titchmarsh $M$-matrix and spectral matrix of the deformation $\tilde{H}_{(\tilde{\mu}, \tilde{\sigma})}$ are computed in terms of the corresponding matrices of the base operator $H$. A variety of additional results and possible extensions, including limit point/limit circle considerations, iterations of isospectral deformations, general Sturm-Liouville operators on arbitrary intervals, and scattering theory, are treated in Section 5. Finally, the single and double commutation methods are reviewed in Appendices A and B, respectively. 


\section{$\S 2$. Preliminaries on the Dirichlet deformation method}

This section sets the stage for a complete spectral characterization of the Dirichlet deformation method in the remainder of this paper.

Suppose

$$
V \in L_{\mathrm{l} x \mathrm{c}}^{1}(\mathbb{R}) \text { is real valued, }
$$

introduce the differential expression $\tau=-d^{2} / d x^{2}+V(x), x \in \mathbb{R}$, and pick $\lambda_{0} \in \mathbb{R}$ and $\eta_{ \pm}\left(\lambda_{0}, x\right)$ satisfying

$$
\begin{gathered}
\tau \dot{\psi}\left(\lambda_{0}\right)=\lambda_{0} \dot{\psi}\left(\lambda_{0}\right) \\
\eta_{ \pm}\left(\lambda_{0}, \cdot\right) \in L^{2}((R, \pm \infty)), \quad R \in \mathbb{R}, \quad \eta_{=}\left(\lambda_{0}, x\right) \text { real-valued }
\end{gathered}
$$

Given $\eta_{ \pm}\left(\lambda_{0}, x\right)$ we define the self-adjoint base (background) operator $H$ in $L^{2}(\mathbb{R})$ via

$$
\begin{aligned}
H f & =\tau f \\
f \in \mathcal{D}(H) & =\left\{g \in L^{2}(\mathbb{R}) \mid g, g^{\prime} \in A C_{\mathrm{loc}}(\mathbb{R}) ; \tau g \in L^{2}(\mathbb{R})\right. \\
& \left.\lim _{x \rightarrow \pm \infty} W\left(\eta_{ \pm}\left(\lambda_{0}\right), g\right)(x)=0 \text { if } \tau \text { is l.c. at } \pm \infty\right\} .
\end{aligned}
$$

Here $W(f, g)(x)=f(x) g^{\prime}(x)-f^{\prime}(x) g(x)$ denotes the Wronskian of $f, g \in A C_{\text {loc }}(\mathbb{R})$ (the set of locally absolutely continuous functions on $\mathbb{R}$ ) and l.p. and l.c. abbreviate the limit point and limit circle cases, respectively. The corresponding boundary condition at $w \infty$ in (2.3) is superfluous and hence to be deleted whenever $\tau$ is l.p. at $\omega \infty, \omega \in\{-,+\}$. The reader unwilling to get caught up in limit circle situations may safely add the assumption that $\tau$ is I.p. at $\pm \infty$ which renders $H$ independent of the choice of $\eta_{ \pm}\left(\lambda_{0}, x\right)$. However, as discussed in Lemma 5.3, assuming $\tau$ to be 1.p. at $\pm \infty$ does not necessarily dispose of all limit circle considerations in connection with the deformation method at hand.

Given $H$ and a fixed reference point $x_{0} \in \mathbb{R}$, we introduce the associated Dirichlet operator $H^{D}$ in $L^{2}(\mathbb{R})$ by

$$
\begin{aligned}
H^{D} f= & \tau f, \\
f \in \mathcal{D}\left(H^{D}\right)= & \left\{g \in L^{2}(\mathbb{R}) \mid g \in A C_{\mathrm{l} \infty}(\mathbb{R}), g^{\prime} \in A C_{\mathrm{loc}}\left(\mathbb{R} \backslash\left\{x_{0}\right\}\right) ; \lim _{\epsilon} g\left(x_{0} \pm \epsilon\right)=0 ;\right. \\
& \left.\tau g \in L^{2}(\mathbb{R}) ; \lim _{x \rightarrow \pm \infty} W\left(\eta_{ \pm}\left(\lambda_{0}\right), g\right)(x)=0 \text { if } \tau \text { is l.c. at } \pm \infty\right\} .
\end{aligned}
$$

Clearly, $H^{D}$ decomposes into

$$
H^{D}=H_{-}^{D} \oplus H_{+}^{D}
$$


with respect to the orthogonal decomposition

$$
L^{2}(\mathbb{R})=L^{2}\left(\left(-\infty, x_{0}\right)\right) \oplus L^{2}\left(\left(x_{0}, \infty\right)\right)
$$

(For notational convenience, we shall later identify $\left(x_{0}, \sigma \infty\right)$ with $\left(-\infty, x_{0}\right)$ or $\left(x_{0}, \infty\right)$ depending on whether $\sigma=-$ or $\sigma=+$.) Moreover, for any $\mu \in$ $\sigma_{d}\left(H^{D}\right) \backslash \sigma(H)\left(\sigma_{d}(\cdot)=\sigma(\cdot) \backslash \sigma_{\text {ess }}(\cdot)\right.$, the discrete spectrum, $\sigma(\cdot)$ and $\sigma_{\text {ess }}(\cdot)$, the spectrum and essential spectrum, respectively), we introduce the Dirichlet datum

$$
(\mu, \sigma) \in\left\{\sigma_{d}\left(H^{D}\right) \backslash \sigma_{d}(H)\right\} \times\{-,+\},
$$

which identifies $\mu$ as a discrete Dirichlet eigenvalue on the interval $\left(x_{0}, \sigma \infty\right)$, that is, $\mu \in \sigma_{d}\left(H_{\sigma}^{D}\right), \sigma \in\{-,+\}$ (but excludes it from being simultaneously a Dirichlet eigenvalue on $\left.\left(x_{0},-\sigma \infty\right)\right)$.

In some cases, for instance, if $V(x) \rightarrow \infty$ as $|x| \rightarrow \infty$, the spectrum and Dirichlet data uniquely determine $V(x)$ [23], Theorem 3.6 (cf. also Remarks 4.9 and 4.10).

Next, we pick a fixed spectral gap $\left(E_{0}, E_{1}\right)$ of $H$, the endpoints of which (without loss of generality) belong to the spectrum of $H$,

$$
\left(E_{0}, E_{1}\right) \subset \mathbb{R} \backslash \sigma(H), \quad E_{0}, E_{1} \in \sigma(H)
$$

and choose a discrete eigenvalue $\mu$ of $H^{D}$ in the closure of that spectral gap,

$$
\mu \in \sigma_{d}\left(H^{D}\right) \cap\left[E_{0}, E_{1}\right]
$$

(we note there is at most one such $\mu$ since $\left(H^{D}-z\right)^{-1}$ is a rank-one perturbation of $\left.(H-z)^{-1}\right)$. According to (2.7), this either gives rise to a Dirichlet datum

$$
(\mu, \sigma) \in\left(E_{0}, E_{1}\right) \times\{-,+\}
$$

or else to a discrete eigenvalue of $H_{-}^{D}$ and $H_{+}^{D}$, that is,

$$
\mu \in\left\{E_{0}, E_{1}\right\} \cap \sigma_{d}(H) \cap \sigma_{d}\left(H_{-}^{D}\right) \cap \sigma_{d}\left(H_{+}^{D}\right)
$$

since the eigenfunction of $H$ associated with $\mu$ has a zero at $x_{0}$. In particular, since $\left(H^{D}-z\right)^{-1}$ is a rank-one perturbation of $(H-z)^{-1}$, one infers

$$
\sigma_{\mathrm{ess}}\left(H^{D}\right)=\sigma_{\mathrm{ess}}(H)
$$

and thus, $\mu \in\left\{E_{0}, E_{1}\right\} \cap \sigma_{\mathrm{ess}}(H)$ is excluded by hypothesis (2.9). Hence, the case distinctions (2.10) and (2.11) are exhaustive. 
In addition to $\mu$ as in (2.9)-(2.11), we also need to introduce $\tilde{\mu} \in\left[E_{0}, E_{1}\right]$ and $\bar{\sigma} \in\{-,+\}$ as follows: Either

$$
(\tilde{\mu}, \sigma) \in\left(E_{0}, E_{1}\right) \times\{-,+\},
$$

or else

$$
\tilde{\mu} \in\left\{E_{0}, E_{1}\right\} \cap \sigma_{d}(H)
$$

Given $H$, we define solutions $\psi_{ \pm}(z, x)$ of $(\tau-z) \psi(z)=0$ which satisfy

$$
\begin{aligned}
& \psi_{ \pm}(z, \cdot) \in L^{2}((R, \pm \infty)), \quad R \in \mathbb{R} \\
& \lim _{x \rightarrow \pm \infty} W\left(\psi_{ \pm}(z), g\right)(x)=0 \quad \text { for all } g \in \mathcal{D}(H) .
\end{aligned}
$$

If $\psi_{ \pm}(z, x)$ exist, they are unique up to constant multiples. In particular, $\psi_{ \pm}(z, x)$ exist for $z \in \mathbb{C} \backslash \sigma_{\text {ess }}(H)$ and we can (and will) assume them to be holomorphic with respect to $z \in \mathbb{C} \backslash \sigma(H)$ and real-valued for $z \in \mathbb{R}$. One can choose,

$$
\psi_{ \pm}(z, x)=\left((H-z)^{-1} \chi_{(a, b)}\right)(x) \quad \text { for } x \begin{aligned}
& >b, \\
& <a,
\end{aligned} \quad-\infty<a<b<\infty
$$

and uniquely continue for $\begin{gathered}<b \\ >a\end{gathered}$. (Here $\chi_{\Omega}(\cdot)$ denotes the characteristic function of a set $\Omega \subseteq \mathbb{R}$.) A finite number of isolated eigenvalues can be included in the domain of holomorphy of $\psi_{ \pm}(z)$ by multiplying (2.16) with an appropriate function of $z$.

Next, we state a simple technical result which will be needed in the context of (2.19) and (2.20).

Lemma 2.1 Let $\psi_{\sigma}(\mu, \cdot), \psi_{\sigma}(\tilde{\mu}, \cdot) \in L^{2}((R, \sigma \infty)), R \in \mathbb{R}$ be defined as in (2.15). Then

$$
\lim _{\tilde{\mu} \rightarrow \mu}(\tilde{\mu}-\mu)^{-1} W\left(\psi_{\sigma}(\mu), \psi_{\sigma}(\tilde{\mu})\right)(x)=-\int_{\sigma \infty}^{x} d x^{\prime} \psi_{\sigma}\left(\mu, x^{\prime}\right)^{2}
$$

\section{Proof Since}

$$
(\tilde{\mu}-\mu)^{-1} W\left(\psi_{\sigma}(\mu), \psi_{\sigma}(\tilde{\mu})\right)(x)=-\int_{\sigma \infty}^{x} d x^{\prime} \psi_{\sigma}\left(\mu, x^{\prime}\right) \psi_{\sigma}\left(\tilde{\mu}, x^{\prime}\right)
$$


$\left((2.18)\right.$ is easily verified by differentiating $W\left(\psi_{\sigma}(\mu), \psi_{\sigma}(\tilde{\mu})\right)(x)$ w.r.t. $x$ and using (2.15)), we only need to justify interchanging the limit $\bar{\mu} \rightarrow \mu$ and the integral in (2.18). By (2.16),

$$
\left((H-\tilde{\mu})^{-1} \chi_{(a, b)}\right)\left(x^{\prime}\right)=c_{ \pm}(\tilde{\mu}) \psi_{ \pm}\left(\tilde{\mu}, x^{\prime}\right) \quad \text { for } \begin{array}{r}
x^{\prime}>x>b>a \\
x^{\prime}<x<a<b
\end{array}
$$

for some constants $c_{ \pm}(\tilde{\mu}) \neq 0$, and hence

$$
-\int_{\sigma \infty}^{x} d x^{\prime} \psi_{\sigma}\left(\mu, x^{\prime}\right) \psi_{\sigma}\left(\tilde{\mu}, x^{\prime}\right)=c_{\sigma}^{-1}\left(\psi_{\sigma}(\mu) \chi_{(x, \sigma \infty)},(H-\tilde{\mu})^{-1} \chi_{(a, b)}\right)
$$

yields the desired continuity with respect to $\tilde{\mu}$. (This fails at first sight if $\mu \in$ $\sigma_{d}(H) \cap \sigma_{d}\left(H^{D}\right)$. A proper factor removes the pole at $z=\mu$ in this case.)

Given $\psi_{\sigma}(\mu, x), \psi_{-\bar{\sigma}}(\tilde{\mu}, x)$, and Lemma 2.1 , we define

$$
W_{(\tilde{\mu}, \tilde{\sigma})}(x)= \begin{cases}(\tilde{\mu}-\mu)^{-1} W\left(\psi_{\sigma}(\mu), \psi_{-\tilde{\sigma}}(\tilde{\mu})\right)(x), & \mu, \tilde{\mu} \in\left[E_{0}, E_{1}\right], \tilde{\mu} \neq \mu \\ -\sigma \int_{\sigma \infty}^{x} d x^{\prime} \psi_{\sigma}\left(\mu, x^{\prime}\right)^{2}, & (\tilde{\mu}, \tilde{\sigma})=(\mu,-\sigma), \mu \in\left(E_{0}, E_{1}\right)\end{cases}
$$

and the associated Dirichlet deformation

$$
\begin{aligned}
\tilde{\tau}_{(\tilde{\mu}, \tilde{\sigma})}= & -\frac{d^{2}}{d x^{2}}+\tilde{V}_{(\tilde{\mu}, \tilde{\sigma})}(x), \\
\tilde{V}_{(\tilde{\mu}, \tilde{\sigma})}(x)= & V(x)-2\left\{\ln \left[W_{(\tilde{\mu}, \tilde{\sigma})}(x)\right]\right\}^{\prime \prime}, \quad x \in \mathbb{R}, \\
& \mu, \tilde{\mu} \in\left[E_{0}, E_{1}\right], \mu \neq \tilde{\mu} \quad \text { or }(\tilde{\mu}, \tilde{\sigma})=(\mu,-\sigma), \mu \in\left(E_{0}, E_{1}\right) .
\end{aligned}
$$

(We will show in Lemma $2 . .2$ that $W_{(\tilde{\mu}, \tilde{\sigma})}(x) \neq 0, x \in \mathbb{R}$ and hence (2.20) is well-defined.) In the remaining cases $(\tilde{\mu}, \tilde{\sigma})=(\mu, \sigma), \mu \in\left[E_{0}, E_{1}\right]$, and $\mu=\tilde{\mu} \in$ $\left\{E_{0}, E_{1}\right\} \cap \sigma_{d}(H)$, we define

$$
\tilde{V}_{(\tilde{\mu}, \tilde{\sigma})}(x)=V(x)
$$

Equation (2.21) represents the trivial deformation of $V(x)$ (i.e., none at all), and for notational simplicity these trivial cases are excluded in the remainder of this paper, unless explicitly stated otherwise. For obvious reasons we will allude to $(2.20)$ as the Dirichlet deformation method in the following.

If $\tilde{\mu} \in \sigma_{d}(H)$, then $\psi_{-}(\tilde{\mu})=c \psi_{+}(\tilde{\mu})$ for some $c \in \mathbb{R} \backslash\{0\}$, showing that $W_{(\tilde{\mu}, \tilde{\sigma})}(x)$, and hence $V_{(\bar{\mu}, \tilde{\sigma})}(x)$ in (2.19) and (2.20), become independent of $\sigma$ or $\tilde{\sigma}$. In this case we shall occasionally use a more appropriate notation and write $\tilde{V}_{\tilde{\mu}}(x)$ and $\tilde{r}_{\tilde{\mu}}$ (instead of $\tilde{V}_{(\tilde{\mu}, \tilde{\sigma})}(x)$ and $\tilde{\tau}_{(\bar{\mu}, \tilde{\sigma})}$ ). 
The next result, taken from [25], shows that (2.20) is well-defined on $\mathbb{R}$. For the reader's convenience, we reproduce the proof of the special case we need of Theorem 1.6 in [25].

Lemma $2.2[25]$ Suppose $\mu, \tilde{\mu} \in\left[E_{0}, E_{1}\right]$ and $\psi_{\sigma}(\mu, x), \psi_{-\sigma}(\tilde{\mu}, x), \sigma, \tilde{\sigma} \in\{-,+\}$ are defined as in (2.15). Then

$$
W\left(\psi_{\sigma}(\mu), \psi_{-\sigma}(\mu)\right)(x) \neq 0, W\left(\psi_{\sigma}(\mu), \psi_{-\sigma}(\tilde{\mu})\right)(x) \neq 0, \quad \mu \neq \tilde{\mu}, x \in \mathbb{R}
$$

and hence,

$$
\tilde{V}_{(\tilde{\mu}, \tilde{\sigma})} \in L_{\mathrm{loc}}^{1}(\mathbb{R})
$$

in (2.20).

Proof Since $W\left(\psi_{\sigma}(\mu), \psi_{-\sigma}(\mu)\right)=$ const. $\neq 0$ and (2.23) is clear from (2.19), (2.20), and (2.22), we only focus on the case $W\left(\psi_{\sigma}(\mu), \psi_{-\bar{\sigma}}(\tilde{\mu})\right)(x) \neq 0, \mu \neq \tilde{\mu}$, $x \in \mathbb{R}$.

First, consider the case $\tilde{\sigma}=\sigma=-$, assume without loss of generality that $\tilde{\mu}>\mu$, and abbreviate

$$
W(x)=W\left(\psi_{-}(\mu), \psi_{-}(\tilde{\mu})\right)(x), \quad x \in \mathbb{R} .
$$

Suppose that

$$
W\left(x_{1}\right)=0 \quad \text { for some } x_{1} \in \mathbb{R} \text {. }
$$

Define

$$
\eta_{1}(x)= \begin{cases}\psi_{-}(\mu, x), & x \leq x_{1}, \\ \gamma_{1} \psi_{+}(\tilde{\mu}, x), & x \geq x_{1},\end{cases}
$$

where $\gamma_{1} \in \mathbb{R}$ is defined such that $\eta_{1} \in \mathcal{D}(H)$ and

$$
\tilde{\eta}_{1}(x)= \begin{cases}\psi_{-}(\mu, x), & x \leq x_{1}, \\ -\gamma_{1} \psi_{+}(\tilde{\mu}, x), & x>x_{1} .\end{cases}
$$

If $\tilde{\mu} \in \sigma_{d}(H)$, we define in addition

$$
\eta_{0}(x)=\psi_{+}(\tilde{\mu}, x)=-\tilde{\eta}_{0}(x), \quad x_{0}=-\infty
$$

and if $\mu \in \sigma_{d}(H)$,

$$
\eta_{2}(x)=\psi_{-}(\mu, x)=\tilde{\eta}_{2}(x), \quad x_{2}=+\infty
$$


Then

$$
\left(\eta_{j}, \eta_{k}\right)=\left(\tilde{\eta}_{j}, \tilde{\eta}_{k}\right) \quad \text { for all } j, k
$$

Indeed, let $j<k$; then (2.25) just means that

$$
\int_{x_{j}}^{x_{k}} d x \psi_{-}(\mu, x) \psi_{+}(\tilde{\mu}, x)=0
$$

But

$$
\int_{x_{1}}^{x_{k}} d x \psi_{-}(\mu, x) \psi_{+}(\tilde{\mu}, x)=(\mu-\tilde{\mu})^{-1}\left[W\left(x_{k}\right)-W\left(x_{j}\right)\right]=0
$$

due to $W\left(x_{1}\right)=0$ and $\lim _{x \rightarrow \pm \infty} W(x)=0$ if $\tilde{\mu}$ or $\mu$ lie in $\sigma_{d}(H)$ since $\lim _{x \rightarrow \pm \infty} W\left(g_{1}, g_{2}\right)(x)=0$ for all $g_{1}, g_{2} \in \mathcal{D}(H)$. (For $x_{0}=-\infty$ take $g_{2}=\psi_{+}(\tilde{\mu}) \in$ $\mathcal{D}(H)$ and choose $g_{1}=\psi_{-}(\mu)$ near $x_{0}=-\infty$ and continue $g_{1} \in \mathcal{D}(H)$ appropriately. Similarly, for $x_{2}=+\infty$, take $g_{1}=\psi_{-}(\mu) \in \mathcal{D}(H)$ and choose $g_{2}=\psi_{+}(\tilde{\mu})$ in a neighborhood of $x_{2}=+\infty$ and continue $g_{2} \in \mathcal{D}(H)$ appropriately.) Next, one verifies that

$$
\left[H-\frac{1}{2}(\tilde{\mu}+\mu)\right] \eta_{j}=\frac{1}{2}(\mu-\tilde{\mu}) \tilde{\eta}_{j}
$$

and hence, for $\eta \in \operatorname{span}\left\{\eta_{j}\right\}$,

$$
\left\|\left[H-\frac{1}{2}(\tilde{\mu}+\mu)\right] \eta\right\|=\frac{1}{2}|\tilde{\mu}-\mu|\|\eta\|
$$

implying

$$
\operatorname{dim} \operatorname{Ran}\left(P_{[\mu, \tilde{\mu}]}(H)\right) \geq \operatorname{dim} \operatorname{span}\left\{\eta_{j}\right\}
$$

where $P_{\Omega}(H)$ denotes the spectral projection of $H$ corresponding to $\Omega \subseteq \mathbb{R}$ But $\psi_{-}(\mu)$ and $\psi_{+}(\tilde{\mu})$ are linearly independent on each interval (since their Wronskian is non-constant) and hence all $\eta_{j}$ are linearly independent. In particular,

$$
\operatorname{dim} \operatorname{Ran}\left(P_{(\mu, \tilde{\mu})}(H)\right) \geq 1
$$

which contradicts our basic hypothesis that $\left(E_{0}, E_{1}\right) \subset \mathbb{R} \backslash \sigma(H)$. This contradiction shows that (2.24) is impossible, and hence $W(x) \neq 0$ for all $x \in \mathbb{R}$.

Next, consider the case $\tilde{\sigma}=-\sigma=-($ and still $\tilde{\mu}>\mu)$. Define

$$
\widehat{W}(x)=W\left(\psi_{\ldots}(\mu), \psi_{-}(\tilde{\mu})\right)(x), \quad x \in \mathbb{R}
$$


and suppose

$$
\widehat{W}\left(x_{1}\right)=0 \quad \text { for some } x_{1} \in \mathbb{R}
$$

We introduce

$$
\eta_{1}(x)= \begin{cases}\psi_{-}(\mu, x)-\gamma_{1} \psi_{-}(\tilde{\mu}, x), & x \leq x_{1} \\ 0, & x \geq x_{1}\end{cases}
$$

(fixing $\gamma_{1}$ by demanding $\eta_{1} \in \mathcal{D}(H)$ ) and

$$
\tilde{\eta}_{1}(x)= \begin{cases}\psi_{-}(\mu, x)+\gamma_{1} \psi_{-}(\tilde{\mu}, x), & x \leq x_{1} \\ 0, & x>x_{1}\end{cases}
$$

If $\tilde{\mu} \in \sigma_{d}(H)$, we introduce in addition

$$
\eta_{0}(x)=\psi_{-}(\tilde{\mu}, x)=-\tilde{\eta}_{0}(x), \quad x_{0}=+\infty
$$

and if $\tilde{\mu} \in \sigma_{d}(H)$,

$$
\eta_{2}(x)=\psi_{-}(\mu, x)=\tilde{\eta}_{2}(x), \quad x_{0}=+\infty
$$

The rest of the proof is analogous to the case considered first: The $\eta_{j}$ 's are linearly independent by considering their supports and

$$
\int_{-\infty}^{x_{1}} d x \psi_{-}(\mu, x) \psi_{-}(\tilde{\mu}, x)=(\mu-\tilde{\mu})^{-1} \lim _{c \downarrow-\infty}\left[\widehat{W}\left(x_{1}\right)-\widehat{W}(c)\right]=0
$$

since $\widehat{W}\left(x_{1}\right)=0$ by hypothesis, and both $\psi_{-}(\mu, x)$ and $\psi_{-}(\tilde{\mu}, x)$ satisfy the boundary conditions of $H$ at $-\infty$.

Finally, the cases $\psi_{+}(\mu, x), \psi_{ \pm}(\tilde{\mu}, x)$ can be obtained by reflection.

Actually, Lemma 2.2 is only the tip of the iceberg. The principal results of [25] relate the number of zeros of appropriate Wronskians on an arbitrary interval $(a, b)$ of the type studied in this section to dimensions of spectral projections of general Sturm-Liouville operators on $(a, b)$. For a previous generalization of Sturm's separation theorem invoking the sign of Wronskians, see [36].

For later reference, we now summarize our basic assumptions on $V, \mu$, and $\tilde{\mu}$ in the following hypothesis.

(H.2.3) (i) Suppose $V \in L_{\text {loc }}^{1}(\mathbb{R})$ to be real-valued. 
(ii)

$$
\begin{aligned}
& \left(E_{0}, E_{1}\right) \subset \mathbb{R} \backslash \sigma(H), \quad E_{0}, E_{1} \in \sigma(H), \\
& \mu \in \sigma_{d}\left(H^{D}\right), \quad(\mu, \sigma) \in\left(E_{0}, E_{1}\right) \times\{-,+\} \quad \text { or } \mu \in\left\{E_{0}, E_{1}\right\} \cap \sigma_{d}(H), \\
& (\tilde{\mu}, \tilde{\sigma}) \in\left(E_{0}, E_{1}\right) \times\{-,+\} \quad \text { or } \tilde{\mu} \in\left\{E_{0}, E_{1}\right\} \cap \sigma_{d}(H), \\
& \mu, \tilde{\mu} \in\left[E_{0}, E_{1}\right], \quad \mu \neq \tilde{\mu} \quad \text { or }(\tilde{\mu}, \tilde{\sigma})=(\mu,-\sigma), \quad \mu \in\left(E_{0}, E_{1}\right) .
\end{aligned}
$$

Next, we introduce various solutions of $\left(\tilde{\tau}_{(\tilde{\mu}, \tilde{\sigma})}-z\right) \tilde{\psi}(z)=0$ needed in (2.32)(2.35) to define the self-adjoint operator $\tilde{H}_{(\tilde{\mu}, \tilde{\sigma})}$ in $L^{2}(\mathbb{R})$ associated with $\tilde{\psi}_{(\tilde{\mu}, \tilde{\sigma})}$. Define

$$
\begin{aligned}
\tilde{\psi}_{-\sigma}(\mu, x) & =\psi_{-\tilde{\sigma}}(\tilde{\mu}, x) / W_{(\tilde{\mu}, \tilde{\sigma})}(x) \\
\tilde{\psi}_{\tilde{\sigma}}(\tilde{\mu}, x) & =\psi_{\sigma}(\mu, x) / W_{(\tilde{\mu}, \tilde{\sigma})}(x), \quad \tilde{\psi}_{\tilde{\sigma}}\left(\tilde{\mu}, x_{0}\right)=0 .
\end{aligned}
$$

Then

$$
\left(\tilde{\tau}_{(\tilde{\mu}, \tilde{\sigma})} \tilde{\psi}_{-\sigma}(\mu)\right)(x)=\mu \tilde{\psi}_{-\sigma}(\mu, x), \quad\left(\tilde{\tau}_{(\tilde{\mu}, \tilde{\sigma})} \tilde{\psi}_{\tilde{\sigma}}(\tilde{\mu})\right)(x)=\tilde{\mu} \tilde{\psi}_{\tilde{\sigma}}(\tilde{\mu}, x)
$$

and

$$
\tilde{\psi}_{-\sigma}(\mu, x) \tilde{\psi}_{\tilde{\sigma}}(\tilde{\mu}, x)=\left[W_{(\tilde{\mu}, \tilde{\sigma})}(x)^{-1}\right]^{\prime}
$$

The Dirichlet deformation operator $\tilde{H}_{(\tilde{\mu}, \tilde{\sigma})}$ associated with $\tilde{\tau}_{(\tilde{\mu}, \tilde{\sigma})}$ in $(2.20)$ is then defined as follows:

$$
\begin{aligned}
& \tilde{H}_{(\tilde{\mu}, \tilde{\sigma})} f=\tilde{\tau}_{(\tilde{\mu}, \tilde{\sigma})} f, \\
& f \in \mathcal{D}\left(\tilde{H}_{(\tilde{\mu}, \tilde{\sigma})}\right)=\left\{g \in L^{2}(\mathbb{R}) \mid g, g^{\prime} \in A C_{\mathrm{loc}}(\mathbb{R}) ; \tilde{\tau}_{(\tilde{\mu}, \tilde{\sigma})} g \in L^{2}(\mathbb{R}) ;\right. \\
& g \text { satisfies one of the b.c.'s in Cases I - III if } \\
& \left.\tilde{\tau}_{(\tilde{\mu}, \tilde{\sigma})} \text { is 1.c. at }-\infty \text { and/or }+\infty\right\} \text {. }
\end{aligned}
$$

The boundary conditions (b.c.'s) alluded to in (2.32) are chosen as follows:

Case I: Either $\tau$ is 1.p. at $\pm \infty$ or $\tilde{\sigma}=\sigma$.

$$
\begin{array}{cc}
\lim _{x \rightarrow \tilde{\sigma} \infty} W\left(\tilde{\psi}_{\tilde{\sigma}}(\tilde{\mu}), g\right)(x)=0 & \text { if } \tilde{\tau}_{(\tilde{\mu}, \tilde{\sigma})} \text { is l.c. at } \tilde{\sigma} \infty \\
\lim _{x \rightarrow-\tilde{\sigma} \infty} W\left(\tilde{\psi}_{-\sigma}(\mu), g\right)(x)=0 & \text { if } \tilde{\tau}_{(\tilde{\mu}, \tilde{\sigma})} \text { is l.c. at }-\tilde{\sigma} \infty .
\end{array}
$$

Case II: $\tilde{\sigma}=-\sigma, \tau$ is 1.c. at $-\infty$ or $+\infty$, and $\mu \in \sigma_{d}(H)$.

$$
\lim _{x \rightarrow \omega \infty} W\left(\tilde{\psi}_{\tilde{\sigma}}(\tilde{\mu}), g\right)(\tilde{x})=0 \quad \text { if } \tilde{\tau}_{(\bar{\mu}, \tilde{\sigma})} \text { is 1.c. at } \omega \infty, \quad \omega \in\{-,+\}
$$


Case III $\tilde{\sigma}=-\sigma, \tau$ is l.c. at $-\infty$ or $+\infty$, and $\tilde{\mu} \in \sigma_{d}(H)$.

$$
\lim _{x \rightarrow \omega \infty} W\left(\tilde{\psi}_{-\sigma}(\mu), g\right)(x)=0 \quad \text { if } \tilde{\tau}_{(\tilde{\mu}, \tilde{\sigma})} \text { is I.c. at } \omega \infty, \quad \omega \in\{-,+\}
$$

(Note that Case II $=$ Case III if $(\tilde{\mu}, \tilde{\sigma})=(\mu,-\sigma)$.)

As always, there is no boundary condition at $\omega \infty$ in (2.32) if $\tilde{\tau}_{(\tilde{\mu}, \tilde{\sigma})}$ is 1.p. at $\omega \infty$, $\omega \in\{-,+\}$. Cases I-III, of course, are not exhaustive. We singled them out since they are the only situations where the spectra of $H$ and $\tilde{H}_{(\tilde{\mu}, \tilde{\sigma})}$ are closely related (see (3.17) and the discussion at the end of Section 3).

If $\tilde{\mu} \in \sigma_{d}(H)$, we will occasionally use the more appropriate notation $\tilde{V}_{\bar{\mu}}(x), \tilde{\tau}_{\bar{\mu}}$, and $\tilde{H}_{\tilde{\mu}}$ (instead of $\tilde{V}_{(\tilde{\mu}, \tilde{\sigma})}(x), \tilde{\tau}_{(\bar{\mu}, \tilde{\sigma})}$, and $\tilde{H}_{(\tilde{\mu}, \tilde{\sigma})}$, cf. the comments following (2.21)).

We conclude this section by introducing the Dirichlet operator $\tilde{H}_{(\tilde{\mu}, \tilde{\sigma})}^{D}$ associated with $\bar{H}_{(\bar{\mu}, \tilde{\sigma})}$ and the fixed reference point $x_{0} \in \mathbb{R}$,

$$
\tilde{H}_{(\tilde{\mu}, \tilde{\sigma})}^{D} f=\tilde{\tau}_{(\tilde{\mu}, \tilde{\sigma})} f
$$

$f \in \mathcal{D}\left(\tilde{H}_{(\tilde{\mu}, \bar{\sigma})}^{D}\right)=\left\{g \in L^{2}(\mathbb{R}) \mid g \in A C_{\mathrm{loc}}(\mathbb{R}), g^{\prime} \in A C_{\mathrm{loc}}\left(\mathbb{R} \backslash\left\{x_{0}\right\}\right) ; \lim _{\epsilon \downarrow 0} g\left(x_{0} \pm \epsilon\right)=0\right.$

$\tilde{\tau}_{(\bar{\mu}, \tilde{\sigma})} g \in L^{2}(\mathbb{R}) ; g$ satisfies one of the b.c.'s in Cases I - III if

$\tilde{\tau}_{(\tilde{\mu}, \tilde{\sigma})}$ is l.c. at $-\infty$ and/or $\left.+\infty\right\}$.

In analogy to $(2.5), \tilde{H}_{(\tilde{\mu}, \tilde{\sigma})}^{D}$ decomposes into

$$
\tilde{H}_{(\tilde{\mu}, \tilde{\sigma})}^{D}=\tilde{H}_{(\tilde{\mu}, \tilde{\sigma}),-}^{D} \oplus \tilde{H}_{(\tilde{\mu}, \tilde{\sigma}),+}^{D}
$$

with respect to (2.6).

\section{§3. Half-line Weyl-Titchmarsh and spectral functions}

In this section we derive the Weyl-Titchmarsh $m$-functions for the Dirichlet deformation operator $\tilde{H}_{(\tilde{\mu}, \tilde{\sigma})}$ and relate them to those of $H$. Moreover, we provide a complete spectral characterization of $\tilde{H}_{(\tilde{\mu}, \tilde{\sigma}), \pm}^{D}$ in terms of $H_{ \pm}^{D}$.

We start by introducing the transformation

$$
U_{(\tilde{\mu}, \tilde{\sigma})}(z):\left\{\begin{array}{l}
A C_{\mathrm{loc}}(\mathbb{R}) \rightarrow L_{\mathrm{loc}}^{1}(\mathbb{R}) \\
f(x) \rightarrow \tilde{f}_{(\tilde{\mu}, \tilde{\sigma})}(z, x)=f(x)-(z-\mu)^{-1} \tilde{\psi}_{-\sigma}(\mu, x) W\left(\psi_{\sigma}(\mu), f\right)(x), \\
z \in \mathbb{C} \backslash\{\mu\}
\end{array}\right.
$$

and note that by inspection,

$\left(\left(\tilde{\tau}_{(\tilde{\mu}, \tilde{\sigma})}-z\right) U_{(\tilde{\mu}, \tilde{\sigma})}(z) \psi(z)\right)(x)=0 \quad$ if and only if $((\tau-z) \psi(z))(x)=0, \quad z \in \mathbb{C} \backslash\{\mu\}$ 
Moreover, one verifies

$$
\begin{aligned}
\tilde{f}_{(\tilde{\mu}, \tilde{\sigma})}(z, x)= & \left(U_{(\tilde{\mu}, \tilde{\sigma})}(z) f\right)(x) \\
= & (z-\mu)^{-1}(z-\tilde{\mu}) f(x)-(z-\mu)^{-1} \psi_{\tilde{\sigma}}(\tilde{\mu}, x) W\left(\psi_{-\tilde{\sigma}}(\mu), f\right)(x), \\
& f \in A C_{\mathrm{loc}}(\mathbb{R}), z \in \mathbb{C} \backslash\{\mu\},
\end{aligned}
$$

$$
\left\{\begin{array}{l}
\left(U_{(\bar{\mu}, \tilde{\sigma})}(\tilde{\mu}) \psi_{-\tilde{\sigma}}(\tilde{\mu})\right)(x)=0 \\
\left(U_{(\bar{\mu}, \tilde{\sigma})}(\tilde{\mu}) \psi_{\tilde{\sigma}}(\tilde{\mu})\right)(x)=(\tilde{\mu}-\mu)^{-1} W\left(\psi_{\tilde{\sigma}}(\bar{\mu}), \psi_{-\tilde{\sigma}}(\tilde{\mu})\right) \tilde{\psi}_{\tilde{\sigma}}(\tilde{\mu}, x) \\
\lim _{z \rightarrow \mu}(z-\mu)\left(U_{(\tilde{\mu}, \tilde{\sigma})}(z) \psi_{\sigma}(\mu)\right)(x)=0 \\
\lim _{z \rightarrow \mu}(z-\mu)\left(U_{(\bar{\mu}, \tilde{\sigma})}(z) \psi_{-\tilde{\sigma}}(\mu)\right)(x)=-W\left(\psi_{\sigma}(\mu), \psi_{-\sigma}(\mu)\right) \tilde{\psi}_{-\sigma}(\mu, x)
\end{array}\right.
$$

In addition, if

$$
(\tau-z) \psi(z)=0, \quad(\tau-\widehat{z}) \widehat{\psi}(\widehat{z})=0, \quad z, \widehat{z} \in \mathbb{C} \backslash\{\mu\}
$$

then

$$
\begin{gathered}
W\left(\tilde{\psi}_{-\sigma}(\mu), U_{(\tilde{\mu}, \tilde{\sigma})}(z) \psi(z)\right)(x)=\frac{W\left(\psi_{-\tilde{\sigma}}(\tilde{\mu}), \psi(z)\right)(x)}{W_{(\tilde{\mu}, \tilde{\sigma})}(x)}, \\
W\left(\tilde{\psi}_{\tilde{\sigma}}(\tilde{\mu}), U_{(\tilde{\mu}, \tilde{\sigma})}(z) \psi(z)\right)(x)=\frac{z-\tilde{\mu}}{z-\mu} \frac{W\left(\dot{\psi}_{\sigma}(\dot{\mu}), \psi(z)\right)(x)}{W_{(\tilde{\mu}, \tilde{\sigma})}(x)}, \\
W\left(U_{(\tilde{\mu}, \tilde{\sigma})}(z) \psi(z), U_{(\tilde{\mu}, \tilde{\sigma})}(\hat{z}) \widehat{\psi}(\hat{z})\right)(x)=\frac{z-\tilde{\mu}}{z-\mu} W(\psi(z), \widehat{\psi}(\widehat{z}))(x) \\
+\frac{z-\widehat{z}}{(z-\mu)(\widehat{z}-\mu)} \frac{W\left(\psi_{\sigma}(\mu), \widehat{\psi}(\widehat{z})\right)(x) W(\psi-\tilde{\sigma}(\tilde{\mu}), \psi(z))(x)}{W_{(\tilde{\mu}, \tilde{\sigma})}(x)} .
\end{gathered}
$$

Next, let $\phi(z, x), \theta(z, x)$ be the standard fundamental system of solutions of $(\tau-z) \psi(z)=0, z \in \mathbb{C}$ defined by

$$
\phi\left(z, x_{0}\right)=\theta^{\prime}\left(z, x_{0}\right)=0, \quad \phi^{\prime}\left(z, x_{0}\right)=\theta\left(z, x_{0}\right)=1, \quad z \in \mathbb{C}
$$

with $x_{0} \in \mathbb{R}$ the reference point used in (2.4), and denote by $\tilde{\theta}_{(\bar{\mu}, \tilde{\sigma})}(z, x), \tilde{\phi}_{(\bar{\mu}, \bar{\sigma})}(z, x)$ the analogous fundamental system of solutions of $\left(\tilde{\tau}_{(\tilde{\mu}, \tilde{\sigma})}-z\right) \tilde{\psi}(z)=0, z \in \mathbb{C}$ satisfying (3.8). Since by definition (2.15), $\psi_{\sigma}(z, \cdot) \in L^{2}((R, \sigma \infty)), R \in \mathbb{R}, z \in$ 
$\mathbb{C} \backslash \mathbb{R}$ satisfy the boundary conditions of $H$ near $\sigma \infty$ (if any) and, in particular, $\lim _{x \rightarrow \sigma \infty} W\left(\psi_{\sigma}(z), g\right)(x)=0, g \in \mathcal{D}(H)$, one obtains

$$
\psi_{\sigma}^{\prime}\left(z, x_{0}\right) / \psi_{\sigma}\left(z, x_{0}\right)=m_{\sigma}(z), \quad \sigma \in\{-,+\}, z \in \mathbb{C} \backslash \mathbb{R}
$$

where $m_{\sigma}(z)$ denotes the Weyl-Titchmarsh $m$-function of $H$ with respect to the half-line $\left(x_{0}, \sigma \infty\right), \sigma \in\{-,+\}$. Thus,

$$
\begin{aligned}
\left(U_{(\tilde{\mu}, \tilde{\sigma})}(z) f\right)\left(x_{0}\right) & =\frac{z-\tilde{\mu}}{z-\mu} f\left(x_{0}\right), \\
\left(U_{(\bar{\mu}, \tilde{\sigma})}(z) f\right)^{\prime}\left(x_{0}\right) & =f^{\prime}\left(x_{0}\right)-\frac{\tilde{\mu}-\mu}{z-\mu} m_{-\tilde{\sigma}}(\tilde{\mu}) f\left(x_{0}\right), \quad z, \tilde{\mu} \in \mathbb{C} \backslash\{\mu\}, f \in A C_{\mathrm{loc}}(\mathbb{R}),
\end{aligned}
$$

imply

$$
\begin{aligned}
\tilde{\phi}_{(\tilde{\mu}, \tilde{\sigma})}(z, x)= & \left(U_{(\tilde{\mu}, \tilde{\sigma})}(z) \phi(z)\right)(x), \\
\tilde{\theta}_{(\tilde{\mu}, \tilde{\sigma})}(z, x)= & \frac{z-\mu}{z-\tilde{\mu}}\left(U_{(\tilde{\mu}, \tilde{\sigma})}(z) \theta(z)\right)(x) \\
& +\frac{\tilde{\mu}-\mu}{z-\tilde{\mu}} m_{-\tilde{\sigma}}(\tilde{\mu})\left(U_{(\tilde{\mu}, \tilde{\sigma})}(z) \phi(z)\right)(x), \quad z, \tilde{\mu} \in \mathbb{C} \backslash\{\mu\} .
\end{aligned}
$$

The case $(\tilde{\mu}, \tilde{\sigma})=(\mu,-\sigma)$ in (3.10) and (3.11) can be obtained by a limiting procedure $\left( \pm m_{ \pm}(z)\right.$ being Herglotz, has at most one simple pole for $\lambda \in\left[E_{0}, E_{1}\right]$ with a negative residue),

$$
\lim _{\tilde{\mu} \rightarrow \mu}(\tilde{\mu}-\mu) m_{\sigma}(\tilde{\mu})=\left(\int_{\sigma \infty}^{x_{0}} d x \phi(\mu, x)^{2}\right)^{-1}, \quad \sigma \in\{-,+\}
$$

(see, e.g., [23], Appendix A for a brief summary on Weyl $m$-functions).

The following general fact on Weyl $m$-functions, which provides an effective tool for computing them in the context of $\tilde{H}_{(\tilde{\mu}, \tilde{\sigma})}$, may well be of independent interest.

Lemma 3.1 Let $\widehat{V} \in L_{\text {loc }}^{1}(\mathbb{R})$ be real-valued, $\widehat{\tau}=-d^{2} / d x^{2}+\widehat{V}(x), x \in \mathbb{R}$, and $\widehat{\eta}_{\sigma}(\lambda, x), \lambda \in \mathbb{R}, \sigma \in\{-,+\}$ non-zero real-valued solutions of $(\widehat{\tau}-\lambda) \widehat{\psi}(\lambda)=0$. Define the self-adjoint operator $\widehat{H}$ in $L^{2}(\mathbb{R})$ by

$$
\begin{aligned}
& \hat{H} f=\widehat{\tau} f \\
& f \in \mathcal{D}(\hat{H})=\left\{g \in L^{2}(\mathbb{R}) \mid g, g^{\prime} \in A C_{\operatorname{loc}}(\mathbb{R}) ; \hat{\tau} g \in L^{2}(\mathbb{R})\right. \\
&\left.\lim _{x \rightarrow \pm \infty} W\left(\widehat{\eta}_{ \pm}(\lambda), g\right)(x)=0 \text { if } \hat{\tau} \text { is l.c. at } \pm \infty\right\} .
\end{aligned}
$$


(If $\widehat{\tau}$ is l.p. at $+\infty$ and/or $-\infty$, the corresponding boundary condition in (3.13) is to be deleted.) Denote by $\widehat{\phi}(z, x), \widehat{\theta}(z, x)$ the fundamental system of solutions of $(\widehat{\tau}-z) \widehat{\psi}(z)=0, z \in \mathbb{C}$, with initial values as in (3.8). Then the limits

$$
\hat{m}_{\sigma}(z)=-\lim _{x \rightarrow \sigma \infty} \frac{W\left(\hat{\eta}_{\sigma}(\lambda), \widehat{\theta}(z)\right)(x)}{W\left(\widehat{\eta}_{\sigma}(\lambda), \widehat{\phi}(z)\right)(x)}, \quad z \in \mathbb{C} \backslash \mathbb{R}, \sigma \in\{-,+\}
$$

exist and represent the Weyl-Titchmarsh $m$-functions of $\hat{H}$ on the half-line $\left(x_{0}, \sigma \infty\right)$.

Proof First suppose that $\widehat{\tau}$ is 1.p. at $\sigma \infty$ and $z \in \mathbb{C} \backslash \mathbb{R}$. Then

$$
\begin{aligned}
-\frac{W\left(\widehat{\eta}_{\sigma}(\lambda), \widehat{\theta}(z)\right)(x)}{W\left(\widehat{\eta}_{\sigma}(\lambda), \widehat{\phi}(z)\right)(x)} & =-\frac{\widehat{\theta}(z, x)+\tan (\beta(x)) \widehat{\theta}^{\prime}(z, x)}{\widehat{\phi}(z, x)+\tan (\beta(x)) \widehat{\phi}^{\prime}(z, x)} \\
\cot (\beta(x)) & =-\eta_{\sigma}^{\prime}(\lambda, x) / \eta_{\sigma}(\lambda, x)
\end{aligned}
$$

converges to $\hat{m}_{\sigma}(z)$. This does not quite represent the typical Weyl limit point consideration in which one usually involves an $x$-independent boundary condition parameter $\beta \in[0, \pi)$. However, due to the 1.p. hypothesis made, the Weyl disks shrink to a limit point and the $x$-dependence of $\beta(x)$ in (3.15) becomes immaterial in the limit $x \rightarrow \sigma \infty$.

Next, assume $\widehat{\tau}$ is l.c. at $\sigma \infty$. Then $\widehat{\phi}(z, \cdot), \widehat{\theta}(z, \cdot) \in L^{2}((R, \sigma \infty)), R \in \mathbb{R}$, and hence the limits

$$
\begin{aligned}
\lim _{x \rightarrow \sigma \infty} W\left(\widehat{\eta}_{\sigma}(\lambda), \widehat{\chi}(z)\right)(x)= & W\left(\widehat{\eta}_{\sigma}(\lambda), \widehat{\chi}(z)\right)\left(x_{0}\right) \\
& +(z-\lambda) \int_{\sigma \infty}^{x_{0}} d x^{\prime} \widehat{\eta}_{\sigma}\left(\lambda, x^{\prime}\right) \widehat{\chi}\left(z, x^{\prime}\right) \text { for } \widehat{\chi}(z, x)=\left\{\begin{array}{l}
\widehat{\phi}(z, x) \\
\widehat{\theta}(z, x)
\end{array}\right.
\end{aligned}
$$

exist. Actually, the limits in (3.16) not only exist but they are also non-zero, since otherwise one could construct self-adjoint operators with boundary conditions at $\sigma \infty$ induced by $\hat{\phi}(z, x)$ or $\hat{\theta}(z, x)$ with associated eigenvalue $z \in \mathbb{C} \backslash \mathbb{R}$.

Next, consider the function

$$
\widehat{\psi}_{\sigma}(z, x)=\hat{\theta}(z, x)+\widehat{m}_{\sigma}(z) \widehat{\phi}(z, x), \quad z \in \mathbb{C} \backslash \mathbb{R}
$$

where $\widehat{m}_{\sigma}(z)$ denotes the $m$-function associated with $\widehat{H}$ on $\left(x_{0}, \sigma \infty\right)$. Since by construction, $\widehat{\psi}_{\sigma}(z, x)$ satisfies the boundary conditions of $\hat{H}$ at $\sigma \infty$, one infers

$$
\begin{aligned}
0 & =\lim _{x \rightarrow \sigma \infty} W\left(\hat{\eta}_{\sigma}(\lambda), \widehat{\psi}_{\sigma}(z)\right)(x) \\
& =\left[\lim _{x \rightarrow \sigma \infty} W\left(\widehat{\eta}_{\sigma}(\lambda), \widehat{\theta}(z)\right)(x)\right]+\widehat{m}_{\sigma}(z)\left[\lim _{x \rightarrow \sigma \infty} W\left(\widehat{\eta}_{\sigma}(\lambda), \widehat{\phi}(z)\right)(x)\right],
\end{aligned}
$$


and hence (3.14) again.

Applying Lemma 3.1 to $\tilde{H}_{(\tilde{\mu}, \tilde{\sigma})}$, we obtain as our first major result the following expression for the half-line Weyl-Titchmarsh $m$-functions of $\tilde{H}_{(\tilde{\mu}, \tilde{\sigma})}$ in terms of those of $H$.

Theorem 3.2 Assume (H.2.3). Given $H$ and $\tilde{H}_{(\tilde{\mu}, \tilde{\sigma})}$ by (2.3) and (2.32), respectively, denote by $m_{ \pm}(z)$ and $\tilde{m}_{(\tilde{\mu}, \tilde{\sigma}), \pm}(z)$ the corresponding $m$-functions associated with the half-lines $\left(x_{0}, \pm \infty\right)$. Then

$$
\begin{aligned}
& \tilde{m}_{(\tilde{\mu}, \tilde{\sigma}), \pm}(z)=\frac{z-\mu}{z-\tilde{\mu}} m_{ \pm}(z)-\frac{\tilde{\mu}-\mu}{z-\tilde{\mu}} m_{-\tilde{\sigma}}(\tilde{\mu}), \quad \tilde{\mu} \neq \mu \\
& \tilde{m}_{(\tilde{\mu}, \tilde{\sigma}), \pm}(z)=m_{ \pm}(z)-\left(\int_{\sigma \infty}^{x_{0}} d x \phi(\mu, x)^{2}\right)^{-1} \frac{1}{z-\mu}, \quad(\tilde{\mu}, \tilde{\sigma})=(\mu,-\sigma), z \in \mathbb{C} \backslash \mathbb{R} .
\end{aligned}
$$

Proof Combining (3.11), (3.5), and (3.6), one computes $\tilde{m}_{(\tilde{\mu}, \tilde{\sigma}), \omega}(z), \omega \in\{-,+\}$ either from

$$
\begin{aligned}
-\lim _{x \rightarrow \omega \infty} \frac{W\left(\tilde{\psi}_{\tilde{\sigma}}(\tilde{\mu}), \tilde{\theta}_{(\tilde{\mu}, \tilde{\sigma})}(z)\right)(x)}{W\left(\tilde{\psi}_{\tilde{\sigma}}(\tilde{\mu}), \tilde{\phi}_{(\tilde{\mu}, \tilde{\sigma})}(z)\right)(x)}= & \frac{z-\mu}{z-\tilde{\mu}}\left[-\lim _{x \rightarrow \omega \infty} \frac{W\left(\psi_{\sigma}(\mu), \theta(z)\right)(x)}{W\left(\psi_{\sigma}(\mu), \phi(z)\right)(x)}\right] \\
& -\frac{\tilde{\mu}-\mu}{z-\tilde{\mu}} m_{-\tilde{\sigma}}(\tilde{\mu})
\end{aligned}
$$

or from

$$
\begin{aligned}
-\lim _{x \rightarrow \omega \infty} \frac{W\left(\tilde{\psi}_{-\sigma}(\mu), \tilde{\theta}_{(\tilde{\mu}, \tilde{\sigma})}(z)\right)(x)}{W\left(\tilde{\psi}_{-\sigma}(\mu), \tilde{\phi}_{(\tilde{\mu}, \tilde{\sigma})}(z)\right)(x)}= & \frac{z-\mu}{z-\tilde{\mu}}\left[-\lim _{x \rightarrow \omega \infty} \frac{W\left(\psi_{-\tilde{\sigma}}(\tilde{\mu}), \theta(z)\right)(x)}{W\left(\psi_{-\tilde{\sigma}}(\tilde{\mu}), \phi(z)\right)(x)}\right] \\
& -\frac{\tilde{\mu}-\mu}{z-\tilde{\mu}} m_{-\tilde{\sigma}}(\tilde{\mu})
\end{aligned}
$$

(or from both), depending on Cases I-III in (2.32)-(2.35) by applying Lemma 3.1 to $\tilde{H}_{(\tilde{\mu}, \tilde{\sigma})}$ and $H$.

An examination of $\tilde{m}_{(\tilde{\mu}, \tilde{\sigma}), \pm}(z)$ in (3.17) then reveals the following behavior near $\mu$ and $\tilde{\mu}$.

Corollary 3.3 (i) Suppose $\mu, \tilde{\mu} \in\left(E_{0}, E_{1}\right), \tilde{\mu} \neq \mu$ or $\mu \in\left\{E_{0}, E_{1}\right\} \cap \sigma_{d}(H)$, $\tilde{\mu} \in\left(E_{0}, E_{1}\right)$. Then, $\tilde{m}_{(\tilde{\mu}, \tilde{\sigma}),-\tilde{\sigma}}(z)$ is holomorphic in a neighborhood of $\tilde{\mu}$ whereas $\tilde{m}_{(\tilde{\mu}, \tilde{\sigma}), \tilde{\sigma}}(z)$ has a simple pole at $\tilde{\mu}$ with residue

$$
\lim _{z \rightarrow \tilde{\mu}}(z-\tilde{\mu}) \tilde{m}_{(\tilde{\mu}, \tilde{\sigma}), \tilde{\sigma}}(z)=(\tilde{\mu}-\mu)\left[\boldsymbol{m}_{\tilde{\sigma}}(\tilde{\mu})-m_{-\tilde{\sigma}}(\tilde{\mu})\right] \neq 0 .
$$

Both $\tilde{m}_{(\tilde{\mu}, \tilde{\sigma}), \pm}(z)$ are holomorphic in a neighborhood of $\mu$. 
(ii) Assume $\mu=\tilde{\mu} \in\left(E_{0}, E_{1}\right), \tilde{\sigma}=-\sigma$. Then $\tilde{m}_{(\tilde{\mu}, \tilde{\sigma}),-\dot{\sigma}}(z)$ is holomorphic in a neighborhood of $\tilde{\mu}$ whereas $\tilde{m}_{(\tilde{\mu}, \tilde{\sigma}), \tilde{\sigma}}(z)$ has a simple pole at $\tilde{\mu}$ with residue

$$
\lim _{z \rightarrow \tilde{\mu}}(z-\tilde{\mu}) \tilde{m}_{(\tilde{\mu}, \tilde{\sigma}), \tilde{\sigma}}(z)=-\lim _{\tilde{\mu} \rightarrow \mu}(\tilde{\mu}-\mu) m_{\sigma}(\tilde{\mu})=-\left(\int_{\sigma \infty}^{x_{0}} d x \phi(\mu, x)^{2}\right)^{-1} .
$$

(iii) Assume $\mu \in\left(E_{0}, E_{1}\right), \tilde{\mu} \in\left\{E_{0}, E_{1}\right\} \cap \sigma_{d}(H)$ or $\mu, \tilde{\mu} \in\left\{E_{0}, E_{1}\right\} \cap \sigma_{d}(H), \mu \neq \tilde{\mu}$. Then $\tilde{m}_{(\tilde{\mu}, \tilde{\sigma}), \pm}(z)$ are both holomorphic in a neighborhood of $\mu$ and $\tilde{\mu}$.

Proof Observing that

$$
\begin{aligned}
& m_{\sigma}(z) \underset{z \rightarrow \mu}{=} c_{\sigma}(z-\mu)^{-1}+O(1), c_{\sigma} \in \mathbb{R} \backslash\{0\}, m_{-\sigma}(z) \underset{z \rightarrow \mu}{=} O(1), \quad \mu \in\left(\lambda_{0}, \lambda_{1}\right) \\
& m_{ \pm}(z) \underset{z \rightarrow \mu}{=} c_{ \pm}(z-\mu)^{-1}+O(1), c_{ \pm} \in \mathbb{R} \backslash\{0\}, c_{ \pm} \lessgtr 0, \mu \in \sigma_{d}\left(H^{D}\right) \cap \sigma(H)
\end{aligned}
$$

cases (i) and (ii) are a straightforward consequence of (3.12), (3.17), and the fact that $m_{-}(\tilde{\mu}) \neq m_{+}(\tilde{\mu})$ since $\tilde{\mu} \notin \sigma_{d}(H)$. For (iii) one observes, in addition, that first,

$$
m_{-}(\bar{\mu})=m_{+}(\tilde{\mu}) \quad \text { since } \tilde{\mu} \in \sigma_{d}(H) \backslash \sigma_{d}\left(H^{D}\right)
$$

by hypothesis, and second,

$$
\lim _{z \rightarrow \lambda}(z-\lambda) m_{ \pm}(z) \quad \text { exists for all } \lambda \in\left[E_{0}, E_{1}\right]
$$

since $\pm m_{ \pm}(z)$ are Herglotz functions (and $\sigma_{\text {ess }}(H) \cap\left[E_{0}, E_{1}\right]=\emptyset$ ).

As we will explore in more detail in the next section, Corollary 3.3 (i) for $\mu, \tilde{\mu} \in\left(E_{0}, E_{1}\right), \mu \neq \tilde{\mu}$ just means the Dirichlet datum $(\mu, \sigma)$ gets changed into $(\tilde{\mu}, \tilde{\sigma})$ and (ii) illustrates the "flip" of the Dirichlet eigenvalue $\mu$ from one halfline $\left(x_{0}, \sigma \infty\right)$ to the other, $\left(x_{0},-\sigma \infty\right)$, changing $(\mu, \sigma)$ into $(\mu,-\sigma)$. The remaining cases represent non-isospectral deformations of $H$ where the eigenvalue $\mu \in \sigma_{d}(H)$, respectively, $\bar{\mu} \in \sigma_{d}(H)$, or both, $\mu, \bar{\mu} \in \sigma_{d}(H)$ are actually "knocked out" of the spectrum of $H$ (i.e., do not belong to $\sigma\left(\tilde{H}_{(\tilde{\mu}, \tilde{\sigma})}\right)$, respectively, $\sigma\left(\tilde{H}_{\tilde{\mu}}\right)$ ).

Corollary 3.4 Let $z \in \mathbb{C} \backslash\{\sigma(H) \cup\{\mu\}\}, \tilde{\mu} \in\left(E_{0}, E_{1}\right)$. Then $\tilde{\psi}_{\tilde{\sigma}}(\tilde{\mu}, x), \tilde{\psi}_{-\sigma}(\mu, x)$, $\mu \neq \tilde{\mu}$, and $\left.\left(U_{(\tilde{\mu}, \tilde{\sigma})}(z)\right) \psi_{ \pm}(z)\right)(x)$ satisfy the boundary conditions of $\tilde{H}_{(\tilde{\mu}, \tilde{\sigma})}$ (if any) at $\tilde{\sigma} \infty,-\sigma \infty$, and $\pm \infty$, respectively. In particular,

$$
\begin{aligned}
\lim _{x \rightarrow \tilde{\sigma} \infty} W\left(\tilde{\psi}_{\sigma}(\tilde{\mu}), \tilde{g}\right)(x) & =0, \\
\lim _{x \rightarrow-\sigma \infty} W\left(\tilde{\psi}_{-\sigma}(\mu), \tilde{g}\right)(x) & =0, \quad \mu \neq \tilde{\mu}, \\
\lim _{x \rightarrow \pm \infty} W\left(U_{(\tilde{\mu}, \tilde{\sigma})}(z) \psi_{ \pm}(z), \tilde{g}\right)(x) & =0
\end{aligned}
$$


for all $\tilde{g} \in \mathcal{D}\left(\tilde{\boldsymbol{H}}_{(\tilde{\mu}, \tilde{\sigma})}\right)$. Moreover,

$$
\begin{aligned}
\dot{\psi}_{\bar{\sigma}}(\tilde{\mu}, \cdot) & \in L^{2}((R, \tilde{\sigma} \infty)), \\
\tilde{\psi}_{-\sigma}(\mu, \cdot) & \in L^{2}((R,-\sigma \infty)), \quad \mu \neq \tilde{\mu} \\
U_{(\tilde{\mu}, \tilde{\sigma})}(z) \psi_{ \pm}(z) & \in L^{2}((R, \pm \infty)), \quad R \in \mathbb{R}
\end{aligned}
$$

(justifying the notation we chose for $\tilde{\psi}_{\tilde{\sigma}}(\tilde{\mu}, x)$ and $\tilde{\psi}_{-\sigma}(\mu, x)$ ).

Proof Let $\dot{H}_{(\bar{\mu}, \tilde{\sigma}), \omega}^{D}$ denote the Dirichlet operators (2.37) corresponding to $\tilde{\tau}_{(\bar{\mu}, \tilde{\sigma})}$ on the half-line $\left(x_{0}, \omega \infty\right), \omega \in\{-,+\}$. Since $\tilde{m}_{(\tilde{\mu}, \tilde{\sigma}), \tilde{\sigma}}(z)$ has a pole at $z=\tilde{\mu}$ by Corollary 3.3, one infers $\tilde{\mu} \in \sigma_{d}\left(\tilde{H}_{(\tilde{\mu}, \bar{\sigma}), \tilde{\sigma}}^{D}\right)$. Moreover, since $\left(\tilde{\tau}_{(\tilde{\mu}, \tilde{\sigma})}-\tilde{\mu}\right) \tilde{\psi}_{\tilde{\sigma}}(\tilde{\mu})=0$ and $\tilde{\psi}_{\tilde{\sigma}}\left(\tilde{\mu}, x_{0}\right)=0$ (cf. (2.29)), $\tilde{\psi}_{\tilde{\sigma}}(\tilde{\mu}, x)$ is the corresponding eigenfunction of $\tilde{H}_{(\tilde{\mu}, \tilde{\sigma}), \tilde{\sigma}}^{D}$ and hence $\tilde{\psi}_{\tilde{\sigma}}(\tilde{\mu}) \in \mathcal{D}\left(\tilde{H}_{(\tilde{\mu}, \bar{\sigma}), \tilde{\sigma}}^{D}\right)$ satisfies (3.25) and (3.26). In the case of $\tilde{\dot{\psi}}_{-\sigma}(\mu, x), \mu \neq \tilde{\mu}$, one verifies that

$$
\tilde{m}_{(\tilde{\mu}, \tilde{\sigma})_{,-\sigma}}(\mu)=m_{-\tilde{\sigma}}(\tilde{\mu})=\dot{\psi}_{-\tilde{\sigma}}^{\prime}\left(\tilde{\mu}, x_{0}\right) / \psi_{-\tilde{\sigma}}\left(\tilde{\mu}, x_{0}\right)=\tilde{\psi}_{-\sigma}^{\prime}\left(\mu, x_{0}\right) / \tilde{\psi}_{-\sigma}\left(\mu, x_{0}\right)
$$

and hence (3.25) and (3.26) are valid for $\tilde{\psi}_{-\sigma}(\mu)$. Finally, as a consequence of (2.15), one infers that

$$
\psi_{ \pm}(z, x)=c_{ \pm}(z)\left[\theta(z, x)+m_{ \pm}(z) \phi(z, x)\right], \quad z \in \mathbb{C} \backslash \sigma(H)
$$

for some constants $c_{ \pm}(z)$. Combining (3.11), (3.17), and applying $U_{(\tilde{\mu}, \tilde{\sigma})}(z)$ to (3.27) results in

$$
\begin{aligned}
\left(\left(U_{(\tilde{\mu}, \tilde{\sigma})}(z) \psi_{ \pm}(z)\right)(x)\right. & =c_{ \pm}(z) \frac{z-\tilde{\mu}}{z-\mu}\left[\tilde{\theta}_{(\tilde{\mu}, \tilde{\sigma})}(z, x)+\tilde{m}_{(\tilde{\mu}, \tilde{\sigma})}(z) \tilde{\phi}_{(\tilde{\mu}, \tilde{\sigma})}(z, x)\right] \\
z & \in \mathbb{C} \backslash\{\sigma(H) \cup\{\mu\}\} .
\end{aligned}
$$

Clearly, (3.28) proves that $U_{(\bar{\mu}, \tilde{\sigma})}(z) \psi_{ \pm}(z)$ satisfy (3.25) and (3.26).

Given the fundamental relation between $\tilde{m}_{(\tilde{\mu}, \tilde{\sigma}), \pm}(z)$ and $m_{ \pm}(z)$ in Theorem 3.2, we can readily derive the ensuing relation between the corresponding spectral functions $\tilde{\rho}_{(\tilde{\mu}, \tilde{\sigma}), \pm}(\lambda)$ and $\rho_{ \pm}(\lambda)$ associated with the half-line Dirichlet operators $\tilde{H}_{(\tilde{\mu}, \tilde{\sigma}), \pm}^{D}$ and $H_{ \pm}^{D}$. The right-continuous non-decreasing functions $\rho_{ \pm}(\lambda)$ and $\tilde{\rho}_{(\tilde{\mu}, \tilde{\sigma}), \pm}(\lambda)$ are defined for $\lambda \in \mathbb{R}$ by

$$
\rho_{ \pm}(\lambda)-\rho_{ \pm}\left(\lambda^{\prime}\right)= \pm \lim _{\delta \downarrow 0} \lim _{\epsilon \downarrow 0} \pi^{-1} \int_{\lambda^{\prime}+\delta}^{\lambda+\delta} d \nu \operatorname{Im}\left\{m_{ \pm}(\nu+i \epsilon)\right\}
$$


and

$$
\tilde{\rho}_{(\bar{\mu}, \bar{\sigma}), \pm}(\lambda)-\tilde{\rho}_{(\tilde{\mu}, \tilde{\sigma}), \pm}\left(\lambda^{\prime}\right)= \pm \lim _{\delta \downarrow} \lim _{\epsilon \downarrow 0} \pi^{-1} \int_{\lambda^{\prime}+\delta}^{\lambda+\delta} d \nu \operatorname{Im}\left[m_{(\bar{\mu}, \tilde{\sigma}) . \pm}(\nu+i \epsilon)\right]
$$

This sets up the second major result of this section.

Theorem 3.5 Assume (H.2.3). Let $H_{ \pm}^{D}$ and $\tilde{H}_{(\tilde{\mu}, \tilde{\sigma}), \pm}^{D}$ denote the Dirichlet operators (2.4) and (2.37), and $d \rho_{ \pm}(\lambda)$ and $d \tilde{\rho}_{(\tilde{\mu}, \bar{\sigma}), \pm}(\lambda)$ the corresponding spectral measures generated by $\rho_{ \pm}(\lambda)$ and $\tilde{\rho}_{(\bar{\mu}, \bar{\sigma}), \pm}(\lambda)$, respectively. Then

$$
\begin{aligned}
& d \tilde{\rho}_{(\tilde{\mu}, \tilde{\sigma}), \pm}(\lambda)=\frac{\lambda-\mu}{\lambda-\tilde{\mu}} d \rho_{ \pm}(\lambda) \\
& +(\tilde{\mu}-\mu)\left\{\begin{array}{ll}
0, & \tilde{\sigma}=\mp \\
{\left[m_{-}(\tilde{\mu})-m_{+}(\tilde{\mu})\right],} & \tilde{\sigma}= \pm
\end{array}\right\} d \theta(\lambda-\tilde{\mu}), \mu \neq \tilde{\mu}, \\
& d \tilde{\rho}_{(\tilde{\mu}, \tilde{\sigma}), \pm}(\lambda)=d \rho_{ \pm}(\lambda)+\left\{\begin{array}{ll}
0, & \sigma= \pm \\
\left( \pm \int_{\mp \infty}^{x_{0}} d x \phi(\mu, x)^{2}\right)^{-1}, & \sigma=\mp
\end{array}\right\} d \theta(\lambda-\mu), \\
& (\tilde{\mu}, \tilde{\sigma})=(\mu,-\sigma), \lambda \in \mathbb{R} .
\end{aligned}
$$

Here $\theta(x)= \begin{cases}1, & x \geq 0, \\ 0, & x<0 .\end{cases}$

Proof Inserting (3.17), for $\mu \neq \tilde{\mu}$ (for simplicity) into (3.30) yields

$$
\begin{aligned}
\tilde{\rho}_{(\bar{\mu}, \bar{\sigma}), \pm}(\lambda)-\tilde{\rho}_{(\tilde{\mu}, \tilde{\sigma}) \pm}\left(\lambda^{\prime}\right) & = \\
\pm \lim _{\delta \downarrow 0} \lim _{\epsilon \downarrow 0} \pi^{-1} \int_{\lambda^{\prime}+\delta}^{\lambda+\delta} d \nu & \frac{\epsilon^{2}+(\nu-\tilde{\mu})(\nu-\mu)}{(\nu-\tilde{\mu})^{2}+\epsilon^{2}} \operatorname{Im}\left[m_{ \pm}(\nu+i \epsilon)\right] \\
& \left.\quad+\frac{(\tilde{\mu}-\mu) \epsilon}{(\nu-\tilde{\mu})^{2}+\epsilon^{2}}\left\{m_{-\tilde{\sigma}}(\tilde{\mu})-\operatorname{Re}\left\{m_{ \pm}(\nu+i \epsilon)\right]\right\}\right\}
\end{aligned}
$$

Since

$$
m_{ \pm}(\nu) \text { are real-valued for } \nu \in\left[E_{0}, E_{1}\right] \backslash\{\mu\}
$$

$$
\operatorname{Im}\left[m_{ \pm}(\nu+i 0)\right] d \nu \text { has no support in a sufficiently small }
$$
neighborhood of $\tilde{\mu}$ (since $\left.\tilde{\mu} \in\left[E_{0}, E_{1}\right] \backslash\{\mu\}\right)$,

$$
\pi^{-1} \frac{\epsilon d \nu}{(\nu-\tilde{\mu})^{2}+\epsilon^{2}} \underset{\epsilon \downarrow 0}{\longrightarrow} d \theta(\nu-\tilde{\mu}) \quad \text { weakly }
$$




$$
\begin{gathered}
\pi^{-1} \operatorname{Im}\left[m_{ \pm}(\nu+i \epsilon)\right] d \nu \underset{\epsilon ! 0}{\longrightarrow} d \rho_{\mp}(\nu) \quad \text { weakly [49], } \\
\lim _{\epsilon ! 0}\left(\mp i \epsilon \pi^{-1} m_{ \pm}(\lambda+i \epsilon)\right)=\rho_{ \pm}(\lambda)-\rho_{ \pm}(\lambda-0) \\
\lim _{\epsilon \downarrow 0}\left(\mp i \epsilon \pi^{-1} m_{ \pm}(\lambda+i \epsilon)\right) \leq \rho_{+}\left(E_{1}\right)-\rho_{ \pm}\left(E_{0}-0\right), \quad \lambda \in\left[E_{0}, E_{1}\right] \\
\left|\epsilon \pi^{-1} m_{ \pm}(\lambda+i \epsilon)\right| \leq C, \quad \epsilon \in\left[0, \epsilon_{0}\right], \lambda \in\left[E_{0}, E_{1}\right] \text { for some } \epsilon_{0}>0, C>0
\end{gathered}
$$

(3.32) implies (3.31) for $\mu \neq \tilde{\mu}$ by splitting the integral in (3.32) into a sufficiently small interval around $\tilde{\mu}$ (if $\tilde{\mu} \in\left[\lambda^{\prime}, \lambda\right]$ ) and the remaining intervals (applying the dominated convergence theorem). The case $(\tilde{\mu}, \tilde{\sigma})=(\mu,-\sigma)$ is treated analogously.

Remark 3.6 If $\tilde{\mu} \neq \mu$, the factor $(\lambda-\mu) /(\lambda-\tilde{\mu}) \neq 1$ in (3.31) shows that the half-line Dirichlet deformation method $H_{ \pm}^{D} \rightarrow \tilde{H}_{(\tilde{\mu}, \tilde{\sigma}), \pm}^{D}$ affects all remaining norming constants corresponding to eigenvalues of $H_{ \pm}^{D}$. More precisely, denote by

$$
c_{ \pm, n}^{2}=\left(\mp \int_{ \pm \infty}^{x_{0}} d x \phi\left(\lambda_{ \pm, n}, x\right)^{2}\right)^{-1}=\rho_{ \pm}\left(\lambda_{ \pm, n}\right)-\rho_{ \pm}\left(\lambda_{ \pm, n}-0\right)
$$

the norming constant associated with $\lambda_{ \pm, n} \in \sigma_{p}\left(H_{ \pm}^{D}\right), \lambda_{ \pm, n} \neq \mu$ and denote by $\tilde{c}_{(\tilde{\mu}, \tilde{\sigma}), \pm, n}^{2}$ the one associated with $\lambda_{ \pm, n} \in \sigma_{p}\left(\tilde{H}_{(\tilde{\mu}, \tilde{\sigma}), \pm}^{D}\right)$. Then

$$
\tilde{c}_{(\tilde{\mu}, \tilde{\sigma}), \pm, n}^{2}=\frac{\lambda_{ \pm, n}-\mu}{\lambda_{ \pm, n}-\tilde{\mu}} c_{ \pm, n}^{2} .
$$

Only in the case $(\tilde{\mu}, \tilde{\sigma})=(\mu,-\sigma)$ do the remaining norming constants stay invariant,

$$
\tilde{c}_{(\mu,-\sigma), \pm, n}^{2}=c_{ \pm, n}^{2}
$$

In fact, the deformation $(\mu, \sigma) \rightarrow(\mu,-\sigma)$ coincides with the isospectral case of the double commutation method considered in Appendix B (cf. Remark B.3(i)). The corresponding invariance in (3.38) was originally proven in [19].

Theorem 3.5 implies the following half-line deformation result.

Theorem 3.7 Assume (H.2.3) and denote by $H_{ \pm}^{D}$ and $\tilde{H}_{(\tilde{\mu}, \tilde{\sigma}), \pm}^{D}$ the half-line Dirichlet operators (2.4) and (2.37). 
(i) Suppose $\mu, \tilde{\mu} \in\left(E_{0}, E_{1}\right)$. Then

$$
\begin{gathered}
\sigma_{(p)}\left(\tilde{H}_{(\tilde{\mu}, \tilde{\sigma}), \tilde{\sigma}}^{D}\right)= \begin{cases}\left\{\sigma_{(p)}\left(H_{\sigma}^{D}\right) \backslash\{\mu\}\right\} \cup\{\tilde{\mu}\}, & \sigma=\tilde{\sigma}, \\
\sigma_{(p)}\left(H_{-\sigma}^{D}\right) \cup\{\tilde{\mu}\}, & \sigma=-\tilde{\sigma},\end{cases} \\
\sigma_{(p)}\left(\tilde{H}_{(\tilde{\mu}, \tilde{\sigma}),-\tilde{\sigma}}^{D}\right)=\left\{\begin{array}{ll}
\sigma_{(p)}\left(H_{\sigma}^{D}\right) \backslash\{\mu\}, & \sigma=-\tilde{\sigma}, \\
\sigma_{(p)}\left(H_{-\sigma}^{D}\right) & \sigma=\tilde{\sigma},
\end{array} \quad \tilde{\mu} \neq \sigma\left(H_{(\tilde{\mu}, \tilde{\sigma}),-\tilde{\sigma}}^{D}\right) .\right.
\end{gathered}
$$

(ii) Assume $\mu \in\left\{E_{0}, E_{1}\right\} \cap \sigma_{d}(H), \tilde{\mu} \in\left(E_{0}, E_{1}\right)$. Then

$$
\begin{aligned}
\sigma_{(p)}\left(\tilde{H}_{(\tilde{\mu}, \tilde{\sigma}), \tilde{\sigma}}^{D}\right) & =\left\{\sigma_{(p)}\left(H_{\tilde{\sigma}}^{D}\right) \backslash\{\mu\}\right\} \cup\{\bar{\mu}\}, \\
\sigma_{(p)}\left(H_{(\tilde{\mu}, \tilde{\sigma}),-\bar{\sigma}}^{D}\right) & =\sigma_{(p)}\left(H_{-\tilde{\sigma}}^{D} \backslash\{\mu\}, \quad \tilde{\mu} \notin \sigma\left(\boldsymbol{H}_{(\tilde{\mu}, \tilde{\sigma}),-\bar{\sigma}}^{D}\right) .\right.
\end{aligned}
$$

(iii) Suppose $\mu \in\left(E_{0}, E_{1}\right), \tilde{\mu} \in\left\{E_{0}, E_{1}\right\} \cap \sigma_{d}(H)$. Then

$$
\begin{aligned}
\sigma_{(p)}\left(\tilde{H}_{\tilde{\mu}, \sigma}^{D}\right) & =\sigma_{(p)}\left(H_{\sigma}^{D}\right) \backslash\{\mu\} \\
\sigma_{(p)}\left(H_{\tilde{\mu},-\sigma}^{D}\right) & =\sigma_{(p)}\left(H_{-\sigma}^{D}\right), \quad \tilde{\mu} \notin \sigma\left(H_{\tilde{\mu}, \pm}^{D}\right)
\end{aligned}
$$

(iv) Assume $\mu, \tilde{\mu} \in\left\{E_{0}, E_{1}\right\} \cap \sigma_{d}(H), \mu \neq \tilde{\mu}$. Then

$$
\sigma_{(p)}\left(\tilde{H}_{\tilde{\mu}, \pm}^{D}\right)=\sigma_{(p)}\left(H_{ \pm}^{D}\right) \backslash\{\mu\}, \quad \tilde{\mu} \notin \sigma\left(\tilde{H}_{\tilde{\mu}, \pm}^{D}\right)
$$

(Here $\sigma_{(p)}(\cdot)$ denotes $\sigma(\cdot)$ or $\sigma_{p}(\cdot)$ (the point spectrum, i.e., the set of eigenvalues) and we recall our occasional use of the notation of $\tilde{H}_{\tilde{\mu}, \pm}^{D}$ instead of $\tilde{H}_{(\tilde{\mu}, \tilde{\sigma}), \pm}^{D}$ if $\tilde{\mu} \in \sigma_{d}(H)$, cf. the paragraph preceding Lemma 2.2.)

(v)

$$
\sigma_{\mathrm{ess}, \mathrm{ac}, \mathrm{sc}}\left(\tilde{H}_{(\tilde{\mu}, \bar{\sigma}), \pm}^{D}\right)=\sigma_{\mathrm{ess}, \mathrm{ac}, \mathrm{sc}}\left(H_{ \pm}^{D}\right) .
$$

Moreover, $\tilde{H}_{(\tilde{\mu}, \tilde{\sigma}), \pm}^{D}$ and $H_{ \pm}^{D}$, restricted to the orthogonal complements of the (at most one-dimensional, possibly equaling $\{0\}$ ) eigenspaces corresponding to $\tilde{\mu}$ and $\mu$, are unitarily equivalent.

Proof This is a direct consequence of Corollary 3.3, Theorem 3.5, and the fact that half-line spectra corresponding to separated boundary conditions are simple. In particular, we note that by Corollary 3.4 (i) and (iii), $\tilde{m}_{(\tilde{\mu}, \tilde{\sigma}), \pm}(z)$ are holomorphic in a sufficiently small neighborhood of $\mu$ and/or $\tilde{\mu}$ whenever they belong to $\sigma_{d}(H)$.

As long as $\mu, \tilde{\mu} \in\left(E_{0}, E_{1}\right)$ and hence $\mu, \tilde{\mu} \notin \sigma_{d}(H)$, (3.39) just says that the Dirichlet datum $(\mu, \sigma)$ associated with $H^{D}=H_{-}^{D} \oplus H_{+}^{D}$ got changed into the Dirichlet datum $(\tilde{\mu}, \tilde{\sigma})$ associated with $\tilde{H}_{(\tilde{\mu}, \tilde{\sigma})}^{D}=\tilde{H}_{(\tilde{\mu}, \tilde{\sigma}),-}^{D} \oplus \tilde{H}_{(\tilde{\mu}, \tilde{\sigma}),+^{-}}^{D}$ The cases (ii)-(iv) 
examine all remaining possibilities where $\mu$ and/or $\tilde{\mu}$ belong to $\sigma_{d}(H)$ and possibly $\sigma_{d}\left(H_{ \pm}^{D}\right)$ in which case, however, they no longer belong to $\sigma_{d}\left(\tilde{H}_{(\tilde{\mu}, \tilde{\sigma})}^{D}\right)$.

We have yet to show that our choices I-III of boundary conditions of $\tilde{H}_{(\tilde{\mu}, \tilde{\sigma})}$ in (2.32)-(2.35) are indeed the only ones that lead to our fundamental formula (3.17) as claimed after (2.35). We only need to focus on l.c. cases and hence assume that $\tilde{\tau}$ is l.c. at $\pm \infty$. By Lemma 3.1 , the $m$-functions $\bar{m}_{(\tilde{\mu}, \tilde{\sigma}), \pm}(z)$ of $\tilde{H}_{(\bar{\mu}, \bar{\sigma})}$ can be computed as follows,

$$
\tilde{m}_{(\tilde{\mu}, \tilde{\sigma}), \pm}(z)=-\lim _{x \rightarrow \pm \infty} \frac{W\left(\tilde{f}(\lambda), \tilde{\theta}_{(\tilde{\mu}, \tilde{\sigma})}(z)\right)(x)}{W\left(\tilde{f}(\lambda), \tilde{\phi}_{(\bar{\mu}, \tilde{\sigma})}(z)\right)(x)}
$$

where $\left(\tilde{\tau}_{(\bar{\mu}, \tilde{\sigma})}-\lambda\right) \tilde{f}(\lambda)=0$ for some $\lambda \in \mathbb{R}$. Consider a corresponding $f(\lambda, x)$ satisfying $(\tau-\lambda) f(\lambda)=0$ and $\tilde{f}(\lambda)=U_{(\tilde{\mu}, \tilde{\sigma})}(\lambda) f(\lambda)$. Then (3.44) becomes

$$
\tilde{m}_{(\tilde{\mu}, \tilde{\sigma}), \pm}(z)=\frac{z-\mu}{z-\tilde{\mu}}\left[-\lim _{x \rightarrow \pm \infty} \frac{W\left(U_{(\tilde{\mu}, \tilde{\sigma})}(\lambda) f(\lambda), U_{(\tilde{\mu}, \tilde{\sigma})}(z) \theta(z)\right)(x)}{W\left(U_{(\tilde{\mu}, \tilde{\sigma})}(\lambda) f(\lambda), U_{(\tilde{\mu}, \tilde{\sigma})}(z) \phi(z)\right)(x)}\right]-\frac{\tilde{\mu}-\mu}{z-\tilde{\mu}} m_{-\tilde{\sigma}}(\tilde{\mu}) .
$$

Applying (3.7) to (3.45) then yields

$$
\begin{aligned}
& \frac{z-\tilde{\mu}}{z-\mu}\left\{\tilde{m}_{(\tilde{\mu}, \tilde{\sigma}), \pm}(z)+\frac{\tilde{\mu}-\mu}{z-\tilde{\mu}} m_{-\tilde{\sigma}}(\tilde{\mu})\right\}=-\lim _{x \rightarrow \pm \infty}\{(\lambda-\tilde{\mu}) W(f(\lambda), \phi(z))(x) \\
& \left.-\frac{(\lambda-z)(\tilde{\mu}-\mu)}{z-\mu} \frac{W\left(\psi_{\sigma}(\mu), \phi(z)\right)(x) W\left(f(\lambda), \psi_{-\sigma}(\tilde{\mu})\right)(x)}{W\left(\psi_{\sigma}(\mu), \psi_{-\tilde{\sigma}}(\tilde{\mu})\right)(x)}\right\} \\
& \left\{(\lambda-\tilde{\mu}) W(f(\lambda), \theta(z))(x)-\frac{(\lambda-z)(\tilde{\mu}-\mu)}{z-\mu} \frac{W\left(\psi_{\sigma}(\mu), \theta(z)\right)(x) W\left(f(\lambda), \psi_{-\sigma}(\tilde{\mu})\right)(x)}{W\left(\psi_{\sigma}(\mu), \psi_{-\tilde{\sigma}}(\tilde{\mu})\right)(x)}\right\} .
\end{aligned}
$$

In order to reproduce (3.17), the right-hand side of (3.46) would have to equal

$$
-\lim _{x \rightarrow \pm \infty} \frac{W(\eta(\lambda), \theta(z))(x)}{W(\eta(\lambda), \phi(z))(x)}=m_{ \pm}(z)
$$

for some real-valued solution $\eta(z, x)$ of $(\tau-\lambda) \psi(\lambda)=0$ which satisfies the boundary conditions of $H$ at $\pm \infty$. Clearly, $f(\lambda, x)=\psi_{\sigma}(\mu, x)$ and $f(\lambda, x)=\psi_{-\bar{\sigma}}(\tilde{\mu}, x)$ are distinguished in (3.46) and these were precisely the cases we singled out in (2.32)(2.35). No other choice of $f(\lambda, x)$ in (3.46) is compatible with (3.47).

\section{$\S 4$. Spectral and Weyl-Titchmarsh matrices, isospectral deforma- tions}

In this section we prove our principal results including explicit computations of the Weyl-Titchmarsh and spectral matrices of $\tilde{H}_{(\tilde{\mu}, \tilde{\sigma})}$ in terms of those of $H$. Moreover, we provide a complete spectral characterization of $\tilde{H}_{(\tilde{\mu}, \tilde{\sigma})}$ and $\tilde{H}_{(\tilde{\mu}, \tilde{\sigma})}^{D}$ in terms of $H$ and $H^{D}$. 
We start with the Weyl-Titchmarsh matrices for $H$ and $\tilde{H}_{(\tilde{\mu}, \tilde{\sigma})}$. To fix notation, we introduce the Weyl-Titchmarsh $M$-matrix in $\mathbb{C}^{2}$ associated with $H$ by (4.1)

$$
\begin{aligned}
& M(z)=\left(M_{p, q}(z)\right)_{1 \leq p, q \leq 2}
\end{aligned}
$$

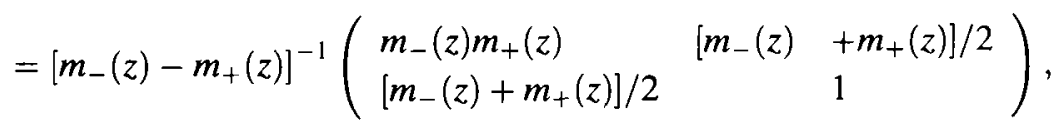

$$
\begin{aligned}
& z \in \mathbb{C} \backslash \mathbb{R}
\end{aligned}
$$

and similarly, in connection with $\tilde{H}_{(\tilde{\mu}, \tilde{\sigma})}$, by

(4.2)

$$
\begin{aligned}
& \tilde{M}_{(\bar{\mu}, \tilde{\sigma})}(z)=\left(\tilde{M}_{(\tilde{\mu}, \bar{\sigma}), p, q}(z)\right)_{1 \leq p, q \leq 2} \\
& =\left[\tilde{m}_{(\tilde{\mu}, \tilde{\sigma}),-}(z)-\tilde{m}_{(\tilde{\mu}, \tilde{\sigma}),+}(z)\right]^{-1} \\
& \times\left(\begin{array}{ll}
\tilde{m}_{(\tilde{\mu}, \tilde{\sigma}),-}(z) \tilde{m}_{(\tilde{\mu}, \tilde{\sigma}),+}(z) & {\left[\tilde{m}_{(\tilde{\mu}, \tilde{\sigma}),-}(z)\right.} \\
{\left[\tilde{m}_{(\tilde{\mu}, \tilde{\sigma}),-}(z)+\tilde{m}_{(\tilde{\mu}, \tilde{\sigma}),+}(z)\right] / 2} & 1
\end{array}\right), \\
& z \in \mathbb{C} \backslash \mathbb{R} \text {. }
\end{aligned}
$$

An application of Theorem 3.2 then yields

Theorem 4.1 Assume $\left(\right.$ H.2.3) and $z \in \mathbb{C} \backslash \mathbb{R}$. Given $H$ and $\tilde{H}_{(\tilde{\mu}, \tilde{\sigma})}$ by (2.3) and (2.32), respectively, their Weyl-Titchmarsh $M$-matrices are related by

$$
\begin{aligned}
\tilde{M}_{(\tilde{\mu}, \tilde{\sigma}), 1,1}(z)= & \frac{z-\mu}{z-\tilde{\mu}} M_{1,1}(z)-2 \frac{\tilde{\mu}-\mu}{z-\tilde{\mu}} m_{-\tilde{\sigma}}(\tilde{\mu}) M_{\mathrm{I}, 2}(z) \\
& \quad+\frac{(\tilde{\mu}-\mu)^{2}}{(z-\mu)(z-\tilde{\mu})} m_{-\tilde{\sigma}}(\tilde{\mu})^{2} M_{2,2}(z), \\
\tilde{M}_{(\tilde{\mu}, \tilde{\sigma}), 1,2}(z)= & M_{1,2}(z)-\frac{\tilde{\mu}-\mu}{z-\mu} m_{-\tilde{\sigma}}(\tilde{\mu}) M_{2,2}(z), \\
\tilde{M}_{(\tilde{\mu}, \tilde{\sigma}), 2,2}(z)= & \frac{z-\tilde{\mu}}{z-\mu} M_{2,2}(z), \quad \tilde{\mu} \neq \mu .
\end{aligned}
$$

Equivalently,

$$
\begin{aligned}
\tilde{M}_{(\tilde{\mu}, \tilde{\sigma})}(z)=(z-\mu)^{-1}(z-\tilde{\mu})^{-1}\left(\begin{array}{ll}
z-\mu & -(\tilde{\mu}-\mu) m_{-\tilde{\sigma}}(\tilde{\mu}) \\
0 & z-\tilde{\mu}
\end{array}\right) \\
\quad \times M(z)\left(\begin{array}{ll}
z-\mu & 0 \\
-(\tilde{\mu}-\mu) m_{-\tilde{\sigma}}(\tilde{\mu}) & z-\tilde{\mu}
\end{array}\right), \quad z \in \mathbb{C} \backslash \mathbb{R}
\end{aligned}
$$

The case $(\tilde{\mu}, \tilde{\sigma})=(\mu,-\sigma)$ follows by a straightforward limiting argument (see (3.12), (3.17), and (3.31)).

Proof This is just a combination of (3.17), (4.1), and (4.2). 
We note that (4.3) can be written as

$$
\tilde{M}_{(\tilde{\mu}, \tilde{\sigma}), 1,1}(z)=\frac{z-\mu}{z-\tilde{\mu}} M_{2,2}(z)\left[m_{+}(z)-\frac{\tilde{\mu}-\mu}{z-\mu} m_{-\tilde{\sigma}}(\tilde{\mu})\right]\left[m_{-}(z)-\frac{\tilde{\mu}-\mu}{z-\mu} m_{-\tilde{\sigma}}(\tilde{\mu})\right] .
$$

A close examination of $\tilde{M}_{(\tilde{\mu}, \tilde{\sigma})}(z)$ then reveals the following behavior near $\mu$ and $\tilde{\mu}$.

Corollary 4.2 $\tilde{M}_{(\tilde{\mu}, \tilde{\sigma})}(z)$ is holomorphic in a neighborhood of $\mu$ and $\tilde{\mu}$ ( for all values of $\mu$ and $\tilde{\mu}$ admitted by (H.2.3)).

Proof It suffices to examine the pole structure (or better, the lack thereof) of $\tilde{M}_{(\tilde{\mu}, \tilde{\sigma}), p, p}(z), p=1,2$ since $\operatorname{det}[\tilde{M}(z)]=-\frac{1}{4}$ then controls that one of $\tilde{M}_{(\tilde{\mu}, \tilde{\sigma}), 1,2}(z)$ as well. The proof then proceeds along a case-by-case study depending on whether $\mu$, respectively $\tilde{\mu}$, lie in $\left(E_{0}, E_{1}\right)$ or in $\left\{E_{0}, E_{1}\right\} \cap \sigma_{d}(H)$. More specifically, one uses (3.26)-(3.28),

$$
\begin{aligned}
& m_{-}(\tilde{\mu})=m_{+}(\tilde{\mu}), M_{2,2}(z) \underset{z \rightarrow \tilde{\mu}}{=} c(z-\tilde{\mu})^{-1}+O(1), \quad c \in \mathbb{R} \backslash\{0\} \\
& \text { if and only if } \tilde{\mu} \in \sigma_{d}(H) \backslash \sigma_{d}\left(H^{D}\right)
\end{aligned}
$$

and

$$
M_{2,2}(z) \underset{z=\mu}{=} c(z-\mu)+O\left((z-\mu)^{2}\right), \quad c \in \mathbb{R} \backslash\{0\} \text { for } \mu \in\left[E_{0}, E_{1}\right] \cap \sigma_{d}\left(H^{D}\right)
$$

The holomorphy assertion then follows directly from (4.5) and (4.7).

Given the basic connection between $\tilde{M}_{(\bar{\mu}, \bar{\sigma})}(z)$ and $M(z)$ in Theorem 4.1 , we can now proceed to derive the analogous relations between the spectral matrices $\tilde{\rho}_{(\tilde{\mu}, \tilde{\sigma})}(\lambda)$ and $\rho(\lambda)$ associated with $\tilde{H}_{(\tilde{\mu}, \tilde{\sigma})}$ and $H$, respectively. The right-continuous non-decreasing matrices $\rho(\lambda)$ and $\tilde{\rho}_{(\tilde{\mu}, \bar{\sigma})}(\lambda)$ in $\mathbb{C}^{2}$ are defined for $\lambda \in \mathbb{R}$ by

$$
\rho(\lambda)=\left(\rho_{p, q}(\lambda)\right)_{1 \leq p, q \leq 2}, \quad \tilde{\rho}_{(\tilde{\mu}, \tilde{\sigma})}(\lambda)=\left(\tilde{\rho}_{(\tilde{\mu}, \tilde{\sigma}), p, q}(\lambda)\right)_{1 \leq p, q \leq 2}
$$

$$
\rho(\lambda)-\rho\left(\lambda^{\prime}\right)=\lim _{\delta \downarrow 0} \lim _{\epsilon \downarrow 0} \pi^{-1} \int_{\lambda^{\prime}+\delta}^{\lambda+\delta} d \nu \operatorname{Im}[M(\nu+i \epsilon)]
$$

$$
\tilde{\rho}_{(\tilde{\mu}, \tilde{\sigma})}(\lambda)-\tilde{\rho}_{(\tilde{\mu}, \tilde{\sigma})}\left(\lambda^{\prime}\right)=\lim _{\delta \downarrow 0} \lim _{\epsilon \downarrow 0} \pi^{-1} \int_{\lambda^{\prime}+\delta}^{\lambda+\delta} d \nu \operatorname{Im}\left[\tilde{M}_{(\tilde{\nu}, \tilde{\sigma})}(\nu+i \epsilon)\right] .
$$

The result for $\tilde{\rho}_{(\tilde{\mu}, \tilde{\sigma})}(\lambda)$ in terms of that of $\rho(\lambda)$ then reads as follows. 
Theorem 4.3 Assume (H.2.3). Given $H$ and $\tilde{H}_{(\tilde{\mu}, \tilde{\sigma})}$ by (2.3) and (2.32), let $d \rho(\lambda)$ and $d \tilde{\rho}_{(\tilde{\mu}, \tilde{\sigma})}(\lambda)$ be the corresponding $\mathbb{C}^{2}$-valued spectral measures generated by $\rho(\lambda)$ and $\tilde{\rho}_{(\tilde{\mu}, \tilde{\sigma})}(\lambda)$, respectively. Then

$$
\begin{gathered}
d \tilde{\rho}_{(\tilde{\mu}, \tilde{\sigma}), 1,1}(\lambda)=\frac{\lambda-\mu}{\lambda-\tilde{\mu}} d \rho_{1,1}(\lambda)-2 \frac{\tilde{\mu}-\mu}{\lambda-\tilde{\mu}} m_{-\tilde{\sigma}}(\tilde{\mu}) d \rho_{1,2}(\lambda) \\
\quad+\frac{(\tilde{\mu}-\mu)^{2}}{(\lambda-\mu)(\lambda-\tilde{\mu})} m_{-\tilde{\sigma}}(\tilde{\mu})^{2} d \rho_{2,2}(\lambda), \\
d \tilde{\rho}_{(\tilde{\mu}, \tilde{\sigma}), 1,2}(\lambda)=d \rho_{1,2}(\lambda)-\frac{\tilde{\mu}-\mu}{\lambda-\mu} m_{-\tilde{\sigma}}(\tilde{\mu}) d \rho_{2,2}(\lambda), \\
d \tilde{\rho}_{(\tilde{\mu}, \tilde{\sigma}), 2,2}(\lambda)=\frac{\lambda-\tilde{\mu}}{\lambda-\mu} d \rho_{2,2}(\lambda), \quad \tilde{\mu} \neq \mu .
\end{gathered}
$$

Equivalently,

$$
\begin{aligned}
d \tilde{\rho}_{(\tilde{\mu}, \tilde{\sigma})}(\lambda)=(\lambda & -\mu)^{-1}(\lambda-\tilde{\mu})^{-1}\left(\begin{array}{ll}
\lambda-\mu & -(\tilde{\mu}-\mu) m_{-\tilde{\sigma}}(\tilde{\mu}) \\
0 & \lambda-\tilde{\mu}
\end{array}\right) \\
& \times d \rho(\lambda)\left(\begin{array}{ll}
\lambda-\mu & 0 \\
-(\tilde{\mu}-\mu) m_{-\tilde{\sigma}}(\tilde{\mu}) & \lambda-\tilde{\mu}
\end{array}\right), \quad \tilde{\mu} \neq \mu .
\end{aligned}
$$

The case $(\tilde{\mu}, \tilde{\sigma})=(\mu,-\sigma)$ follows by a straightforward limiting argument (cf. (3.12), (3.17), and (3.31)).

Proof It suffices to consider $\tilde{\rho}_{(\bar{\mu}, \tilde{\sigma}), 2,2}(\lambda)$, the remaining cases being analogous. Equation (4.5) and

$$
\begin{aligned}
\operatorname{Im}\left[\tilde{M}_{(\tilde{\mu}, \tilde{\sigma}), 2,2}(\nu+i \epsilon)\right]= & \frac{\epsilon^{2}+(\nu-\mu)(\nu-\tilde{\mu})}{(\nu-\mu)^{2}+\epsilon^{2}} \operatorname{Im}\left[M_{2,2}(\nu+i \epsilon)\right] \\
& +\frac{(\tilde{\mu}-\mu) \epsilon}{(\nu-\mu)^{2}+\epsilon^{2}} \operatorname{Re}\left[M_{2,2}(\nu+i \epsilon)\right]
\end{aligned}
$$

show that one can follow the proof of Theorem 3.5 step by step involving (3.34)(3.36) (replacing $m_{ \pm}(z), \rho_{ \pm}(\lambda)$ by $M_{2,2}(z), \rho_{2,2}(\lambda)$, etc.).

This finally leads to the principal spectral deformation result of this paper.

Theorem 4.4 Assume (H.2.3) and let $H, \tilde{H}_{(\tilde{\mu}, \tilde{\sigma})}, H^{D}$, and $\tilde{H}_{(\tilde{\mu}, \tilde{\sigma})}^{D}$ be defined by (2.3), (2.32), (2.4), and (2.36), respectively.

(i) Suppose $\mu, \tilde{\mu} \in\left(E_{0}, E_{1}\right)$. Then $\tilde{H}_{(\tilde{\mu}, \tilde{\sigma})}$ and $H$ are unitarily equivalent. Moreover, $\tilde{H}_{(\tilde{\mu}, \tilde{\sigma})}^{D}$ and $H^{D}$, restricted to the orthogonal complements of the onedimensional eigenspaces corresponding to $\tilde{\mu}$ and $\mu$, are unitarily equivalent. 
(ii) Assume $\mu \in\left\{E_{0}, E_{1}\right\} \cap \sigma_{d}(H), \tilde{\mu} \in\left(E_{0}, E_{1}\right)$. Then

$$
\begin{aligned}
& \sigma_{(p)}\left(\tilde{H}_{(\tilde{\mu}, \tilde{\sigma})}\right)=\sigma_{(p)}(H) \backslash\{\mu\}, \\
& \sigma_{(p)}\left(\tilde{H}_{(\tilde{\mu}, \tilde{\sigma})}^{D}\right)=\left\{\sigma_{(p)}\left(H^{D}\right) \backslash\{\mu\}\right\} \cup\{\tilde{\mu}\} .
\end{aligned}
$$

(iii) Suppose $\mu \in\left(E_{0}, E_{1}\right), \tilde{\mu} \in\left\{E_{0}, E_{1}\right\} \cap \sigma_{d}(H)$. Then

$$
\begin{aligned}
\sigma_{(p)}\left(\tilde{H}_{\tilde{\mu}}\right) & =\sigma_{(p)}(H) \backslash\{\tilde{\mu}\}, \\
\sigma_{(p)}\left(\tilde{H}_{\tilde{\mu}}^{D}\right) & =\sigma_{\langle p\rangle}\left(H^{D}\right) \backslash\{\mu\}, \quad \tilde{\mu} \notin \sigma\left(\tilde{H}_{\tilde{\mu}}^{D}\right) .
\end{aligned}
$$

(iv) Assume $\mu, \tilde{\mu} \in\left\{E_{0}, E_{1}\right\} \cap \sigma_{d}(H), \mu \neq \tilde{\mu}$. Then

$$
\begin{aligned}
\sigma_{(p)}\left(\tilde{H}_{\tilde{\mu}}\right) & =\sigma_{(p)}(H) \backslash\left\{E_{0}, E_{1}\right\} \\
\sigma_{(p)}\left(\tilde{H}_{\tilde{\mu}}^{D}\right) & =\sigma_{(p)}\left(H^{D}\right) \backslash\{\mu\}, \quad \tilde{\mu} \notin \sigma\left(\tilde{H}_{\tilde{\mu}}^{D}\right)
\end{aligned}
$$

In cases (ii)-(iv), the corresponding pairs of operators, restricted to the obvious orthogonal complements of the eigenspaces corresponding to $\mu$ and/or $\bar{\mu}$, are unitarily equivalent. In particular,

$$
\sigma_{\text {ess,ac,sc }}\left(\tilde{H}_{(\bar{\mu}, \tilde{\sigma})}\right)=\sigma_{\text {ess,ac,sc }}\left(\tilde{H}_{(\tilde{\mu}, \tilde{\sigma})}^{D}\right)=\sigma_{\text {ess,ac,sc }}\left(H^{D}\right)=\sigma_{\text {ess,ac,sc }}(H)
$$

Proof This is a direct consequence of Corollary 3.3, Theorems 3.5, 3.7, and 4.4, and the orthogonal decompositions of $H^{D}=H_{-}^{D} \oplus H_{+}^{D}, \tilde{H}_{(\tilde{\mu}, \bar{\sigma})}^{D}=\tilde{H}_{(\tilde{\mu}, \bar{\sigma}),-}^{D} \oplus \tilde{H}_{(\bar{\mu}, \bar{\sigma}),+}^{D}$. Moreover, in connection with case (iv), one observes that $\mu \in \sigma_{d}(H) \cap \sigma_{d}\left(H^{D}\right)$ necessarily implies that $\tilde{\mu} \in\left\{\left\{E_{0}, E_{1}\right\} \cap \sigma_{d}(H)\right\} \backslash\{\mu\}$ cannot lie in $\sigma_{d}\left(H^{D}\right)$ (i.e., two consecutive discrete eigenvalues of $H$ cannot both belong to the spectrum of $H^{D}$ ).

Remark 4.5 Perhaps the most spectacular consequence of Theorem 4.4(i), from an inverse spectral point of view, is the fact that any finite number of deformations of Dirichlet data within spectral gaps of $V$ yields a potential $\tilde{V}$ in the isospectral class of $V$. No further constraints on $\left(\mu_{j}, \sigma_{j}\right),\left(\tilde{\mu}_{j}, \tilde{\sigma}_{j}\right)$, other than $\left(\mu_{j}, \sigma_{j}\right),\left(\tilde{\mu}_{j}, \tilde{\sigma}_{j}\right) \in$ $\left(E_{j-1}, E_{j}\right) \times\{-,+\},\left(E_{j-1}, E_{j}\right) \in \mathbb{R} \backslash \sigma(H), j=1, \ldots, N, N \in \mathbb{N}$ are involved.

On an intuitive level, the Dirichlet deformation method amounts to the following two-step procedure. In the first commutation step, effected by $\psi_{\sigma}(\mu, x)$ in (2.19), the Dirichlet eigenvalue $\mu \in\left(E_{0}, E_{1}\right)$ associated with $H=-d^{2} / d x^{2}+V$ on the interval $\left(x_{0}, \sigma \infty\right)$ for some $x_{0}=x_{0}(\mu) \in \mathbb{R}$ is moved to $\infty$, thereby producing a singular intermediate potential deformation of $V(x)$ in the process. The second commutation step, effected by $\psi_{-\tilde{\sigma}}(\tilde{\mu}, x)$ in (2.19), then moves back this "Dirichlet eigenvalue" from $\infty$ to $\bar{\mu} \in\left(E_{0}, E_{1}\right)$ associated with the interval $\left(x_{0}, \tilde{\sigma} \infty\right)$. In 
the latter process, the resulting deformation $\tilde{V}_{(\tilde{\mu}, \tilde{\sigma})}(x)$ becomes regular again (i.e., $\left.W_{(\tilde{\mu}, \tilde{\sigma})}(x) \neq 0, x \in \mathbb{R}\right)$ and isospectral to the original base potential $V(x)$.

We conclude this section with a series of remarks. A variety of additional results and possible extensions in connection with the Dirichlet deformation method will be presented in Section 5 .

Remark 4.6 (i) The isospectral property (i) in Theorem 4.4, in the special case of periodic potentials $V(x)$, has first been proven by Finkel, Isaacson, and Trubowitz [12]. Further results can be found in Buys and Finkel [4] and Iwasaki [32] (see also [40]). Similar constructions in connection with Schrödinger operators on a compact interval can be found in Pöschel and Trubowitz [48] and Ralston and Trubowitz [49] (see our discussion in the introduction).

(ii) By inspection, Dirichlet deformations produce the commuting diagram

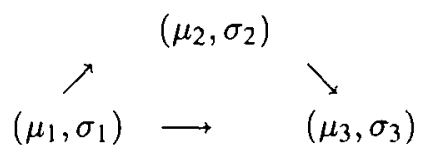

for $\left(\mu_{j}, \sigma_{j}\right) \in\left[E_{0}, E_{1}\right] \times\{-,+\}, 1 \leq j \leq 3$ according to (H.2.3).

(iii) Let $\mu \in\left(E_{0}, E_{1}\right)$. Then the (isospectral) Dirichlet deformation $(\mu, \sigma) \rightarrow$ $(\mu,-\sigma)$ is precisely the isospectral case of the double commutation method considered in Appendix B (see Remark B.3(i)). It simply flips the Dirichlet eigenvalue $\mu$ on the half-line $\left(x_{0}, \sigma \infty\right)$ to the other half-line $\left(x_{0},-\sigma \infty\right)$. In the special case where $V(x)$ is periodic, this procedure has first been used by McKean and van Moerbeke [46].

(iv) In analogy to Remark 3.6, the Dirichlet deformation method as displayed in (4.12)-(4.14) changes magnitudes of discontinuities of $\rho(\lambda)$ at all eigenvalues $\lambda_{n} \in \sigma_{p}(H)$ as long as $\bar{\mu} \neq \mu$. Even in the special case $(\tilde{\mu}, \tilde{\sigma})=(\mu,-\sigma)$ discussed in item (iii) above, one obtains invariance of the magnitudes of jumps at $\lambda_{n}$ only for the spectral matrix element $\rho_{2,2}(\lambda)$.

(v) In the non-isospectral cases (ii)-(iv), a combination of the present Dirichlet deformation method with the double commutation method in Appendix B can restore isospectrality by inserting an eigenvalue at $\mu, \tilde{\mu}$, or both.

Remark 4.7 In certain cases where the base (background) potential $V$ is reflectionless (cf. (5.6)) and $H$ is bounded from below and has no singularly continuous spectrum, the isospectral class Iso( $V)$ of $V$ (the set of all $\tilde{V}$ 's such that $\sigma(\tilde{H})=\sigma(H)$ ) is completely characterized by the distribution of Dirichlet (initial) data $\left(\mu_{j+1}\left(x_{0}\right), \sigma_{j+1}\left(x_{0}\right)\right) \in\left[E_{j}, E_{j+1}\right] \times\{-,+\}, j \in J$ in non-trivial spectral gaps of $H$. Here $x_{0} \in \mathbb{R}$ is a fixed reference point and $J=\{0,1, \ldots, N-1\}, N \in \mathbb{N}$ or $j \in J=\mathbb{N}_{0}$ $(=\mathbb{N} \cup\{0\})$ parametrizes these non-trivial spectral gaps $\left(E_{j}, E_{j+1}\right)$ of $H$ (the trivial 
one being $(-\infty$, inf $\sigma(H)))$. Prime examples of this type are periodic potentials, algebro-geometric quasi-periodic finite-gap potentials, and certain limiting cases thereof (e.g., soliton potentials). In these cases, an iteration of the Dirichlet deformation method, in the sense that $\left(\mu_{j+1}\left(x_{0}\right), \sigma_{j+1}\left(x_{0}\right)\right) \rightarrow\left(\tilde{\mu}_{j+1}\left(x_{0}\right), \tilde{\sigma}_{j+1}\left(x_{0}\right)\right)$ within $\left[E_{j}, E_{j+1}\right] \times\{-,+\}$ for each $j \in J$, independently of each other (cf. (5.4), (5.5)) yields an explicit realization of the underlying isospectral class Iso $(V)$ of potentials with base $V$. In the periodic case, this has first been proved by Finkel, Isaacson, and Trubowitz [12] (see also [4], [32]). More precisely, the inclusion of limiting cases $\mu_{j+1}\left(x_{0}\right) \in\left\{E_{j}, E_{j+1}\right\} \cap \sigma_{\text {ess }}(H)$ requires a special argument (since it is excluded by (H.2.3)) but this can be provided in the special cases at hand.

Remark 4.8 Another case of primary interest concerns potentials $V$ with purely discrete spectra bounded from below, that is,

$$
\begin{gathered}
\sigma(H)=\sigma_{d}(H)=\left\{E_{j}\right\}_{j \in \mathbb{N}_{0}}, \quad-\infty<E_{0}, E_{j}<E_{j+1}, j \in \mathbb{N}_{0}, \\
\sigma_{\mathrm{ess}}(H)=\emptyset .
\end{gathered}
$$

(For simplicity, one may think in terms of the harmonic oscillator $V(x)=x^{2},[38]$, [45].) In this case, either

$$
\left(\mu_{j+1}\left(x_{0}\right), \sigma_{j+1}\left(x_{0}\right)\right) \in\left(E_{j}, E_{j+1}\right) \times\{-,+\}
$$

or

$$
\mu_{j+1}\left(x_{0}\right)=E_{j+1}=\mu_{j+2}\left(x_{0}\right),
$$

that is, Dirichlet eigenvalues necessarily meet in pairs whenever they hit an eigenvalue of $H$. The following trace formula for $V(x)$ in terms of $\sigma(H)=\left\{E_{j}\right\}_{j \in \mathbb{N}_{0}}$ and $\sigma\left(H_{x}^{D}\right)=\left\{\mu_{j}(x)\right\}_{j \in \mathbb{N}}\left(H_{y}^{D}\right.$ the Dirichlet operator associated with $\tau=-d^{2} / d x^{2}+V(x)$ and an additional Dirichlet boundary condition at $x=y$ ), proven in [24],

$$
V(x)=E_{0}+\lim _{\alpha \downarrow 0} \alpha^{-1} \sum_{j=1}^{\infty}\left(2 e^{-\alpha \mu_{j}(x)}-e^{-\alpha E_{j}}-e^{-\alpha E_{j+1}}\right),
$$

then shows one crucial difference to the periodic-type cases mentioned previously. Unlike in the periodic case, though, the initial Dirichlet eigenvalues $\mu_{j+1}\left(x_{0}\right)$ cannot be prescribed arbitrarily in the spectral gaps $\left(E_{j}, E_{j+1}\right)$ of $H$. Indeed, the fact that the Abelian regularization in the trace formula (4.23) for $V(x)$ converges to a limit restricts the asymptotic distribution of $\mu_{j+1}(x) \in\left[E_{j}, E_{j+1}\right]$ as $j \rightarrow \infty$. However, as stressed in Remark 4.5, one of the fundamental consequences of this paper concerns the fact that there is no such restriction for any finite number of spectral gaps of $H$ (see (5.4), (5.5)). In other words, only the tail end of the Dirichlet 
eigenvalues $\mu_{j+1}\left(x_{0}\right)$ as $j \rightarrow \infty$ is restricted (the precise nature of this restriction being unknown at this point), any finite number of them can be placed arbitrarily in the spectral gaps $\left(E_{j}, E_{j+1}\right)$ (with the obvious "crossing" restrictions at the common boundary $E_{j+1}$ of $\left(E_{j}, E_{j+1}\right)$ and $\left.\left(E_{j+1}, E_{j+2}\right)\right)$. This fact served as one of our prime motivations for this paper. The only other known restriction to date on Dirichlet initial data $\left(\mu_{j}\left(x_{0}\right), \sigma_{j}\left(x_{0}\right)\right)$ is that $\sigma_{j}\left(x_{0}\right)=-$ and $\sigma_{j}\left(x_{0}\right)=+$ infinitely often, that is, both half-lines $\left(-\infty, x_{0}\right)$ and $\left(x_{0}, \infty\right)$ support (naturally) infinitely many Dirichlet eigenvalues.

The general characterization of the full isospectral class of operators with purely discrete spectra remains a (very interesting) open problem. It is quite surprising that more than sixty years after the founding of quantum mechanics, the isospectral class of the one-dimensional harmonic oscillator remains shrouded in mystery.

Finally, it seems appropriate to comment on the map from $V$ to Dirichlet data alluded to in the introduction and describe the role played by the additional parameter needed in the Dirichlet data in the special case where eigenvalues of $H^{D}$ and $H$ coincide.

Remark 4.9 Suppose $H$ (and hence $H^{D}$ ) has empty essential spectrum and is bounded from below. In order to show that the map from $V$ to Dirichlet data (suitable interpreted to allow for eigenvalue coincidences of $H^{D}$ and $H$ ) is one-one when defined on the isospectral set of $V$, one can use results in [23] and [24] as follows. Since the spectra of both $H$ and $H^{D}$ are purely discrete they determine the diagonal Green's function $G\left(z, x_{0}, x_{0}\right)=\left[m_{-}(z)-m_{+}(z)\right]^{-1}$ by formula (6.7) of [24]. Moreover, since the Weyl $m$-functions are meromorphic, we only need to know whether the pole of $G\left(z, x_{0}, x_{0}\right)^{-1}$ at each $z=\mu$ belongs to $m_{+}(z)$ or $m_{-}(z)$ in order to recover $m_{ \pm}(z)$, that is, we need $\sigma$ as in (2.7). If $\mu$ is an eigenvalue of $H^{D}$ and $H$, and hence a pole of $m_{-}(z)$ and $m_{+}(z), \sigma$ is not merely a sign but needs to contain the information about how the residue of $G\left(z, x_{0}, x_{0}\right)^{-1}$ at $z=\mu$ is distributed between $m_{+}(z)$ and $m_{-}(z)$ as discussed in Theorem 3.6 of [23]. A convenient choice for this additional parameter (see, e.g., [20]) would be $\sigma=\left(\gamma_{+}-\gamma_{-}\right) /\left(\gamma_{+}+\gamma_{-}\right) \in(-1,1)$, where $\gamma_{ \pm}$denote the respective residues of $m_{ \pm}(z)$ at $z=\mu$. In this extended sense (when compared to (2.7)) the spectrum of $H$ and the Dirichlet data $(\mu, \sigma)$ uniquely determine $V(x)$ for a.e. $x \in \mathbb{R}$. These considerations are not confined to operators with purely discrete spectra but also apply to situations where $H$ is reflectionless and has no singularly continuous spectrum. This has been discussed in the context of Jacobi operators in [20] but analogous arguments work in the Schrödinger operator case.

Remark 4.10 The additional parameter $\sigma_{0}$ introduced in Remark 4.9 in the case where $E_{0}$ is an eigenvalue of $H$ and $H^{D}$ (and both have purely discrete spectra) can be tuned to produce all corresponding isospectral potentials in Iso $(V)$. In fact, the double commutation procedure (see Appendix B) allows one to add/subtract 
$\tilde{\gamma}_{1}$ to the residues of the Weyl $m$-functions (see (B.27)) as long as the term under the logarithm in (B.14) does not become zero.

In addition, one can use the following three-step procedure to generate a prescribed degeneracy at an eigenvalue $E_{0}$ of $H$ :

(i) Use the Dirichlet deformation method to move $\mu$ to a discrete eigenvalue $E_{0}$ of $H$. (This removes both the discrete eigenvalue $E_{0}$ of $H$ and the (Dirichlet) eigenvalue $\mu$ of $H^{D}$.)

(ii) As a consequence of step (i), there is now another eigenvalue $\tilde{\mu}$ of $H^{D}$ in the resulting larger spectral gap of $H$. Move $\tilde{\mu}$ to $E_{0}$ using the Dirichlet deformation method.

(iii) Use the double commutation method to insert an eigenvalue of $H$ at $E_{0}$.

Finally, use the method at the beginning of this remark to change $\sigma_{0}$ into any allowed value.

Theorems 3.7 and B.2 then show that the resulting operator is unitarily equivalent to the original operator $H$, and (3.17) and (B.27) then prove that the remaining Dirichlet eigenvalues remain invariant.

\section{$\S 5$. Various additional results and possible extensions}

In our final section we discuss a variety of additional results and sketch possible extensions, including limit point/limit circle considerations, iterations of the Dirichlet deformation procedure, scattering theory, and general Sturm-Liouville operators on arbitrary intervals.

We start with two limit point results. The first, although trivial from a technical point of view, nevertheless will apply in a great variety of situations.

Lemma 5.1 Assume (H.2.3) and define $H$ and $H_{ \pm}^{D}$ as in (2.3) and (2.4). Let $\sigma \in\{-,+\}$ and suppose that one of the following conditions (i)-(iii) holds.

(i) $\sigma_{\mathrm{ess}}\left(\boldsymbol{H}_{\sigma}^{D}\right) \neq \emptyset$.

(ii) $\sigma_{\mathrm{ess}}\left(H_{\sigma}^{D}\right)=\emptyset$ and $H_{\sigma}^{D}$ is bounded from below.

(iii) $\sigma\left(H_{\sigma}^{D}\right)=\sigma_{d}\left(H_{\sigma}^{D}\right)=\left\{E_{\sigma, n}\right\}_{n \in \mathbb{Z}}$ with $\sum_{n \in \mathbb{Z}}\left(1+E_{\sigma, n}^{2}\right)^{-1}=\infty$.

Then, both $\tau$ and $\tilde{\tau}_{(\tilde{\mu}, \tilde{\sigma})}$ are in the limit point case at $\sigma \infty$.

Proof Clearly, $\tau$ is l.p. at $\sigma \infty$ if condition (i) holds since differential expressions being regular at $x_{0}$ and l.c. at $\sigma \infty$ can only generate self-adjoint operators in $L^{2}\left(\left(x_{0}, \sigma \infty\right)\right)$ with purely discrete spectra. (Indeed, all solutions of $\tau \psi=z \psi, z \in \mathbb{C}$ being in $L^{2}\left(\left(x_{0}, \sigma \infty\right)\right)$ yield a compact, in fact, Hilbert-Schmidt resolvent.) These spectra necessarily accumulate at $+\infty$ and $-\infty$ (see, e.g., Lemma C.1 in [19] for a short argument based on principal solutions in Hartman's terminology). Finally, the Hilbert-Schmidt argument for the resolvent would lead to $\sum_{n \in \mathbb{Z}}\left(1+E_{n}^{2}\right)^{-1}<\infty$ for the corresponding eigenvalues $\left\{E_{n}\right\}_{n \in \mathbb{Z}}$ in the l.c. case at $\sigma \infty$. Theorem 3.7 then 
shows that $\tilde{H}_{(\tilde{\mu}, \bar{\sigma}), \sigma}^{D}$ shares the corresponding property (i), (ii), or (iii), rendering $\tilde{\tau}_{(\tilde{\mu}, \tilde{\sigma})}$ l.p. at $\sigma \infty$ as well.

Our second limit point result is a bit more refined and tailored toward the Dirichlet deformation method (denoted as DDM for brevity in the remainder of this section).

Lemma 5.2 In addition to (H.2.3), assume that $\mu, \tilde{\mu} \in\left(E_{0}, E_{1}\right), \mu \neq \bar{\mu}$ and $\tilde{\sigma}=\sigma$. Then $\tilde{\tau}_{(\bar{\mu}, \sigma)}$ is in the limit point (resp., limit circle) case at $\omega \infty$ if and only if $\tau$ is limit point (resp., limit circle) at $\omega \infty, \omega \in\{-,+\}$.

Proof Assume that $\tau$ is l.p. at $\omega \infty$ and suppose the contrary for $\tilde{\tau}_{(\tilde{\mu}, \sigma)}$, that is, suppose $\tilde{\tau}_{(\tilde{\mu}, \sigma)}$ is 1.c. at $\omega \infty$ and hence

$$
\tilde{\psi}_{\sigma}(\tilde{\mu}, \cdot), \tilde{\psi}_{-\sigma}(\mu, \cdot) \in L^{2}\left(\left(x_{0}, \omega \infty\right)\right)
$$

Since by hypothesis, $\tau$ is l.p. at $\omega \infty$, both functions in (5.1) generate the same $m$ function $\tilde{m}_{(\tilde{\mu}, \bar{\sigma}), \omega}(z)$ associated with $\tilde{H}_{(\tilde{\mu}, \tilde{\sigma})}$ on $\left(x_{0}, \omega \infty\right)$. This follows directly from (3.18) and (3.19). In particular, both $\bar{\psi}_{\sigma}(\tilde{\mu}, x)$ and $\psi_{-\sigma}(\mu, x)$ fulfill the boundary conditions of $\tilde{H}_{(\bar{\mu}, \bar{\sigma})}$ and the analog of (3.25) at $\omega \infty$. As a consequence of (5.1), we obtain existence, in fact, vanishing of the limit

$$
\lim _{x \rightarrow \omega \infty} W\left(\tilde{\psi}_{\sigma}(\tilde{\mu}), \tilde{\psi}_{-\sigma}(\mu)\right)(x)=0
$$

Since by Corollary $3.4 \tilde{\psi}_{\sigma}(\tilde{\mu}, \cdot) \in L^{2}\left(\left(x_{0}, \sigma \infty\right)\right)$ and $\tilde{\psi}_{-\sigma}(\mu, \cdot) \in L^{2}\left(\left(x_{0},-\sigma \infty\right)\right)$ satisfy

$$
\lim _{x \rightarrow \sigma \infty} W\left(\tilde{\psi}_{\sigma}(\tilde{\mu}), \tilde{g}\right)(x)=0, \quad \lim _{x \rightarrow-\sigma \infty} W\left(\tilde{\psi}_{-\sigma}(\tilde{\mu}), \tilde{g}\right)(x)=0
$$

for all $\tilde{g} \in \mathcal{D}\left(\tilde{H}_{(\tilde{\mu}, \sigma)}\right)$, we arrive at the following case distinction. Either

(i) $\omega=\sigma$. Then $\tilde{\psi}_{-\sigma}(\mu) \in \mathcal{D}\left(\tilde{H}_{(\tilde{\mu}, \sigma)}\right)$ and hence $\mu \in \sigma_{p}\left(\tilde{H}_{(\tilde{\mu}, \sigma)}\right)$, or

(ii) $\omega=-\sigma$. Then $\tilde{\psi}_{\sigma}(\tilde{\mu}) \in \mathcal{D}\left(\tilde{H}_{(\tilde{\mu}, \sigma)}\right)$ and hence $\bar{\mu} \in \sigma_{p}\left(\tilde{H}_{(\bar{\mu}, \sigma)}\right)$.

Either way, since $\sigma\left(\tilde{H}_{(\tilde{\mu}, \sigma)}\right)=\sigma(H)$ by Theorem 4.4(i), we get a contradiction since by hypothesis $\mu, \tilde{\mu} \in\left(E_{0}, E_{1}\right) \subset \mathbb{R} \backslash \sigma(H)$. If $\tau$ is l.c. at $\omega \infty$, suppose $\tilde{\tau}_{(\tilde{\mu}, \bar{\sigma})}$ is l.p. at $\omega \infty$. By studying the reverse deformation $(\tilde{\mu}, \sigma) \rightarrow(\mu, \sigma), \tau$ would have to be l.p. at $\omega \infty$ by our previous argument. This contradiction shows $\tilde{\tau}_{(\tilde{\mu}, \sigma)}$ is l.c. at $\omega \infty$. By symmetry in $\tau$ and $\tilde{\tau}_{(\bar{\mu}, \sigma)}$, the proof is complete.

After these encouraging results, we shall take a chance (and possibly disappoint the reader) by describing a construction showing that DDM in general neither respects the l.c. nor the l.p. case if $\tilde{\sigma}=-\sigma$. More precisely, we will construct an example where we "hop" from l.c. to l.p. and then back to a 1.c. case. This illustrates our warning raised in the paragraph following (2.3). 
Lemma 5.3 If $\tilde{\sigma}=-\sigma$, the Dirichlet deformation method (as presented by (H.2.3), (2.3), and (2.32)), in general, neither preserves the limit point nor limit circle case.

Proof Let $\mu \in\left(E_{0}, E_{1}\right)$ and choose $H$ in such a way that $\tau$ is 1.c. at $\sigma \infty$ but l.p. at $-\sigma \infty$ by assuming $\sigma_{\text {ess }}\left(H_{-\sigma}^{D}\right) \neq \emptyset$. Now consider the (sign flip) deformation $(\mu, \sigma) \rightarrow(\mu,-\sigma)$. Clearly, $\tilde{\tau}_{(\mu,-\sigma)}$ is l.p. at $-\sigma \infty$ since $\sigma_{\text {ess }}\left(\tilde{H}_{(\mu, \sigma),-\sigma}^{D}\right)=$ $\sigma_{\text {ess }}\left(\boldsymbol{H}_{-\sigma}^{D}\right) \neq \emptyset$. However, we claim that $\tilde{\tau}_{(\mu,-\sigma)}$ is 1.p. at $\sigma \infty$ as well. To prove this assertion, we suppose the contrary, that is, we assume $\tilde{\tau}_{(\mu,-\sigma)}$ to be l.c. at $\sigma \infty$. Then the left-hand side of the following identity (cf. (2.17))

$$
\tilde{\psi}_{-\sigma}(\mu, x) \tilde{\psi}_{-\sigma}(\mu, x)=-\frac{d}{d x}\left(\int_{\sigma \infty}^{x} d x^{\prime} \psi_{\sigma}\left(\mu, x^{\prime}\right)^{2}\right)^{-1}
$$

is in $L^{1}\left(\left(x_{0}, \sigma \infty\right)\right)$. However, the right-hand side is clearly not integrable near $\sigma \infty$, providing the desired contradiction. Hence, $\tilde{\tau}_{(\mu,-\sigma)}$ is indeed l.p. at $\pm \infty$. A further sign flip, that is, $(\mu,-\sigma) \rightarrow(\mu, \sigma)$, restores the original differential expression $\tau$ which was l.c. at $\sigma \infty$ (see Remark 4.6(ii)). Summarizing,

$$
\tau \longrightarrow \tilde{\tau}_{(\mu,-\sigma)} \longrightarrow\left(\tilde{\tau}_{(\mu,-\sigma)}\right)_{(\mu, \sigma)}=\tau
$$

that is, in obvious notation,

$$
\underset{\text { l.c. }}{(\mu, \sigma)} \rightarrow(\mu,-\sigma) \rightarrow(\mu, \sigma)
$$

displays the required deformations.

By Remark 4.6(ii) again, (5.3) can be modified to an example of the type

$$
\underset{\text { l.p. }}{(\mu, \sigma)} \underset{\text { l.c. }}{(\tilde{\mu},-\sigma)}, \quad \mu, \tilde{\mu} \in\left(E_{0}, E_{1}\right), \mu \neq \tilde{\mu}
$$

using the chain

$$
\underset{\text { l.p. }}{(\mu, \sigma)} \underset{\text { l.p. }}{(} \underset{\mu}{\text { l.c. }}(\tilde{\mu})
$$

(relying on Lemma 5.2 in the first step).

Next, we briefly comment on how to iterate DDM (see [4], [12]). Suppose $V \in L_{\text {loc }}^{1}(\mathbb{R})$ and

$$
\left(E_{n}, E_{n+1}\right), \quad \mu_{n+1}, \tilde{\mu}_{n+1} \in\left[E_{n}, E_{n+1}\right], \quad \sigma_{n+1}, \tilde{\sigma}_{n+1} \in\{-,+\}
$$


satisfy (H.2.3) for each $n=0,1, \ldots, N-1, N \in \mathbb{N}$. Then the Dirichlet deformation result after $N$ steps, denoted by $\tilde{\tau}_{\left(\tilde{\mu}_{1}, \tilde{\sigma}_{1}\right), \ldots,\left(\tilde{\mu}_{N}, \tilde{\sigma}_{N}\right)}$, reads as follows:

$$
\begin{aligned}
\tilde{\tau}_{\left(\tilde{\mu}_{1}, \tilde{\sigma}_{1}\right), \ldots,\left(\tilde{\mu}_{N}, \tilde{\sigma}_{N}\right)} & =-\frac{d^{2}}{d x^{2}}+\tilde{V}_{\left(\tilde{\mu}_{1}, \tilde{\sigma}_{1}\right), \ldots,\left(\tilde{\mu}_{N}, \tilde{\sigma}_{N}\right)}(x), \\
\tilde{V}_{\left(\tilde{\mu}_{1}, \tilde{\sigma}_{1}\right), \ldots,\left(\tilde{\mu}_{N}, \tilde{\sigma}_{N}\right)}(x) & =V(x)-2\left\{\ln \left[W_{\left(\tilde{\mu}_{1}, \tilde{\sigma}_{1}\right), \ldots,\left(\tilde{\mu}_{N}, \tilde{\sigma}_{N}\right)}(x)\right]\right\}^{\prime \prime}, \quad x \in \mathbb{R}
\end{aligned}
$$

$$
\begin{array}{r}
W_{\left(\tilde{\mu}_{1}, \tilde{\sigma}_{1}\right), \ldots,\left(\tilde{\mu}_{N}, \tilde{\sigma}_{N}\right)}(x)=\frac{W\left(\dot{\psi}_{\sigma_{1}}\left(\mu_{1}\right), \psi_{-\dot{\sigma}}\left(\tilde{\mu}_{1}\right), \ldots, \psi_{\sigma_{N}}\left(\mu_{N}\right), \psi_{-\bar{\sigma}_{N}}\left(\tilde{\mu}_{N}\right)\right)(x)}{\left(\tilde{\mu}_{1}-\mu_{1}\right) \ldots\left(\tilde{\mu}_{N}-\mu_{N}\right)} \\
\mu_{n+1}, \tilde{\mu}_{n+1} \in\left[E_{n}, E_{n+1}\right], \mu_{n} \neq \tilde{\mu}_{n}, 0 \leq n \leq N-1 .
\end{array}
$$

In case $\left(\tilde{\mu}_{n_{0}}, \tilde{\sigma}_{n_{0}}\right)=\left(\mu_{n_{0}},-\sigma_{n_{0}}\right)$ for some $0 \leq n_{0} \leq N-1$, one amends (5.5) by replacing the pair $\left(\tilde{\mu}_{n_{0}}-\mu_{n_{0}}\right)^{-1}\left(\psi_{\sigma_{n_{0}}}\left(\tilde{\mu}_{n_{0}}\right), \psi_{-\tilde{\sigma}_{n_{0}}}\left(\tilde{\mu}_{n_{0}}\right)\right)$ by $\left(\psi_{\sigma_{n_{0}}}\left(\mu_{n_{0}}\right), \dot{\psi}_{\sigma_{n_{0}}}\left(\mu_{n_{0}}\right)\right)$ (where "." abbreviates $d / d \lambda$ ). It should perhaps be pointed out that $W\left(\psi_{\sigma_{1}}\left(\mu_{1}\right), \ldots, \psi_{-\tilde{\sigma}_{N}}\left(\tilde{\mu}_{N}\right)\right)(x)$ in (5.5) denotes a slightly modified $2 N \times 2 N$ Wronskian of solutions of $\tau \psi(z)=z \psi(z)$. In particular, it is understood that $\psi^{\prime \prime}(z, x)$ must be replaced by $(V(x)-z) \psi(z, x)$, and similarly for higher derivatives of $\psi$. At the end of this process only $\psi, \psi^{\prime}$, and $V$ enter (5.5) and no additional smoothness on $V$ is required. At this point each of our previous results has an obvious counterpart in connection with (5.4), (5.5).

Next, we will show that DDM leaves reflectionless potentials invariant. We recall that $H$ (resp. $V$ ) is called reflectionless if and only if

(5.6) for all $x \in \mathbb{R}, \quad \arg (G(\lambda+i 0, x, x))=\pi / 2 \quad$ for (Lebesgue) a.e. $\lambda \in \sigma_{\mathrm{ess}}(H)$.

Here $G\left(z, x, x^{\prime}\right)$ denotes the Green's function of $H$ (i.e., the integral kernel of $\left.(H-z)^{-1}\right)$ and $G(\lambda+i 0, x, x)=\lim _{\epsilon \downarrow 0} G(\lambda+i \epsilon, x, x)$ in obvious notation. As discussed in [21], (5.6) is equivalent to

$$
m_{+}(\lambda+i 0)=\overline{m_{-}(\lambda+i 0)} \text { for a.e. } \lambda \in \sigma_{\mathrm{ess}}(H)
$$

This then implies

Lemma 5.4 Assume (H.2.3). Then $\tilde{H}_{(\tilde{\mu}, \tilde{\sigma})}$, is reflectionless if and only if $H$ is.

Proof By (3.17) and Theorem 4.4, one observes that (5.7) holds if and only if

$$
\tilde{m}_{(\bar{\mu}, \tilde{\sigma}),+}(\lambda+i 0)=\overline{\tilde{m}_{(\tilde{\mu}, \tilde{\sigma}),-}(\lambda+i 0)} \quad \text { for a.e. } \lambda \in \sigma_{\mathrm{ess}}\left(\tilde{H}_{(\bar{\mu}, \tilde{\sigma})}\right)=\sigma_{\mathrm{ess}}(H)
$$

Since

$$
G\left(z, x_{0}, x_{0}\right)=\left[m_{-}(z)-m_{+}(z)\right]^{-1}
$$


we might add the fact that by (4.2) and (4.5),

$$
\tilde{G}_{(\bar{\mu}, \tilde{\sigma})}\left(z, x_{0}, x_{0}\right)=\frac{z-\tilde{\mu}}{z-\mu} G\left(z, x_{0}, x_{0}\right)
$$

where $\tilde{G}_{(\tilde{\mu}, \tilde{\sigma})}\left(z, x, x^{\prime}\right)$ denotes the Green's function of $\tilde{H}_{(\tilde{\mu}, \tilde{\sigma})}$. (5.9) again underscores the change $\mu \rightarrow \tilde{\mu}$ (but it stops short of indicating $\sigma \rightarrow \tilde{\sigma}$ ).

In the following we describe how to define DDM for general Sturm-Liouville operators. To keep this section within a reasonable length, we only point out the major changes required in this context.

$$
\begin{aligned}
& V \in L_{\mathrm{loc}}^{1}(\mathbb{R}) \text { real-valued, } x_{0} \in \mathbb{R} \rightarrow p^{-1}, q, k \in L_{\mathrm{loc}}^{1}((a, b)), \quad k p \in A C_{\mathrm{loc}}((a, b)) \text {, } \\
& p>0, k>0, q \text { real-valued, } \\
& -\infty \leq a<b \leq \infty, x_{0} \in(a, b) \text {, } \\
& f^{\prime} \rightarrow p f^{\prime} \\
& W(f, g)(x) \rightarrow \widehat{W}(f, g)(x)=f(x) p(x) g^{\prime}(x)-p(x) f^{\prime}(x) g(x), \\
& L^{2}(\mathbb{R}) \rightarrow L^{2}((a, b) ; k d x), \\
& \tau \rightarrow \widehat{\tau}=\frac{1}{k(x)}\left(-\frac{d}{d x} p(x) \frac{d}{d x}+q(x)\right), \quad x \in(a, b), \\
& \tilde{\tau}_{(\tilde{\mu}, \tilde{\sigma})} \rightarrow \widetilde{\widehat{\tau}}_{(\tilde{\mu}, \tilde{\sigma})}=\frac{1}{k(x)}\left(-\frac{d}{d x} p(x) \frac{d}{d x}+\tilde{q}_{(\tilde{\mu}, \tilde{\sigma})}(x)\right), \\
& \tilde{q}_{(\tilde{\mu}, \tilde{\sigma})}(x)=q(x)+\left(\frac{1}{k(x)}(k(x) p(x))^{\prime}-2 \frac{d}{d x} p(x)\right) \frac{d}{d x} \ln \left[\widehat{W}_{(\tilde{\mu}, \tilde{\sigma})}(x)\right] \text {, } \\
& \widehat{W}_{(\tilde{\mu}, \tilde{\sigma})}(x)= \begin{cases}(\tilde{\mu}-\mu)^{-1} \widehat{W}\left(\psi_{\sigma}(\mu), \psi_{-\bar{\sigma}}(\tilde{\mu})\right)(x), & \mu, \tilde{\mu} \in\left[E_{0}, E_{1}\right], \mu \neq \tilde{\mu} \\
-\sigma \int_{\sigma \infty}^{x} k\left(x^{\prime}\right) d x^{\prime} \psi_{\sigma}\left(\mu, x^{\prime}\right)^{2}, & (\tilde{\mu}, \tilde{\sigma})=(\mu,-\sigma), \mu \in\left(E_{0}, E_{1}\right),\end{cases} \\
& \tau \psi_{\sigma}(\mu)=\mu \psi_{\sigma}(\mu), \quad \tau \psi_{-\tilde{\sigma}}(\tilde{\mu})=\tilde{\mu} \psi_{-\bar{\sigma}}(\tilde{\mu}) .
\end{aligned}
$$

Since (a generalization of) Lemma 2.2 is actually proven in [25] for the general Sturm-Liouville case on $(a, b)$, every result in this paper extends to the general setting in (5.10). In particular, the fundamental Theorems 3.2, 3.5, 3.7, 4.1, 4.3, and 4.4 (replacing $\phi\left(\mu, x^{\prime}\right)^{2}$ by $k\left(x^{\prime}\right) \phi\left(\mu, x^{\prime}\right)^{2}$ if $(\tilde{\mu}, \tilde{\sigma})=(\mu,-\sigma)$ ) do not change at all.

Next, we turn to short-range scattering. Assuming temporarily

$$
V \in L^{1}(\mathbb{R},(1+|x|) d x) \text { to be real-valued, }
$$


the Jost solutions $f_{ \pm}(z, x)$ associated with $V$ are defined as usual by

$$
\begin{gathered}
f_{ \pm}(z, x)=e^{+i z^{1 / 2} x}+\int_{ \pm \infty}^{x} d x^{\prime} z^{-1 / 2} \sin \left[z^{1 / 2}\left(x-x^{\prime}\right)\right] V\left(x^{\prime}\right) f_{ \pm}\left(z, x^{\prime}\right), z \in \mathbb{C} \backslash\{0\} \\
\operatorname{Im}\left(z^{1 / 2}\right) \geq 0
\end{gathered}
$$

\section{Denoting}

$$
f_{ \pm}(\lambda, x)=\lim _{\epsilon \downarrow 0} f_{ \pm}(\lambda+i \epsilon, x), \quad \lambda>0
$$

one obtains

$$
f_{ \pm}(\lambda, x)=\frac{1}{T(\lambda)} \overline{f_{\mp}(\lambda, x)}+\frac{R^{\ell}(\lambda)}{T(\lambda)} f_{\mp}(\lambda, x), \quad \lambda>0
$$

and

$$
T(\lambda)=\frac{2 i \lambda^{1 / 2}}{W\left(f_{-}(\lambda), f_{+}(\lambda)\right)}
$$

$$
R^{\ell}(\lambda)=-\frac{W\left(\overline{f_{-}(\lambda)}, f_{+}(\lambda)\right)}{W\left(f_{-}(\lambda), f_{-}(\lambda)\right)}, \quad R^{r}(\lambda)=-\frac{W\left(f_{-}(\lambda), \overline{f_{+}(\lambda)}\right)}{W\left(f_{-}(\lambda), f_{+}(\lambda)\right)}, \quad \lambda>0
$$

define the scattering matrix $S(\lambda)$ in $\mathbb{C}^{2}$ associated with the pair $\left(H, H_{0}\right)$, where $H_{0}=-d^{2} / d x^{2}, \mathcal{D}\left(H_{0}\right)=H^{2,2}(\mathbb{R})$,

$$
S(\lambda)=\left(\begin{array}{ll}
T(\lambda) & R^{r}(\lambda) \\
R^{\ell}(\lambda) & T(\lambda)
\end{array}\right), \quad \lambda>0
$$

(5.12) and (5.14) then yield (see, e.g., [8], Section 2)

$$
f_{ \pm}(\lambda, x)= \begin{cases}e^{ \pm i \lambda^{1 / 2} x}+o(1), & x \rightarrow \pm \infty, \\ \frac{1}{T(\lambda)} e^{ \pm i \lambda^{1 / 2} x}+\frac{R^{\varepsilon}(\lambda)}{T(\lambda)} e^{\mp i \lambda^{1 / 2} x}+o(1), & x \rightarrow \mp \infty,\end{cases}
$$

$$
f_{ \pm}^{\prime}(\lambda, x)=\left\{\begin{array}{ll} 
\pm i \lambda^{1 / 2} e^{ \pm i \lambda^{1 / 2} x}+o(1), & x \rightarrow \pm \infty, \\
\frac{ \pm i \lambda^{1 / 2}}{T(\lambda)} e^{ \pm i \lambda^{1 / 2} x} \mp i \lambda^{1 / 2} \frac{R^{\prime}(\lambda)}{T(\lambda)} e^{\mp i \lambda^{1 / 2} x}+o(1), & x \rightarrow \mp \infty,
\end{array} \quad \lambda>0 .\right.
$$


The following result proves that DDM preserves the class of $L^{1}(\mathbb{R} ;(1+|x|) d x)$ potentials and computes the scattering matrix $\tilde{S}_{(\bar{\mu}, \tilde{\sigma})}(\lambda)$,

$$
\tilde{S}_{(\tilde{\mu}, \tilde{\sigma})}(\lambda)=\left(\begin{array}{cc}
\tilde{T}_{(\tilde{\mu}, \tilde{\sigma})}(\lambda) & \tilde{R}_{(\bar{\mu}, \tilde{\sigma})}^{r}(\lambda) \\
\tilde{R}_{(\tilde{\mu}, \tilde{\sigma})}^{\ell}(\lambda) & \tilde{T}_{(\tilde{\mu}, \tilde{\sigma})}(\lambda)
\end{array}\right), \quad \lambda>0,
$$

associated with the pair $\left(\tilde{H}_{(\tilde{\mu}, \bar{\sigma})}, H_{0}\right)$ in terms of $S(\lambda)$ in $(5.16)$ associated with $\left(H, H_{0}\right)$.

Lemma 5.5 In addition to (H.2.3), assume $\mu, \tilde{\mu} \in\left(E_{0}, E_{1}\right) \subset(-\infty, 0)$. Then $\tilde{V}_{(\tilde{\mu}, \tilde{\sigma})} \in L^{\prime}(\mathbb{R} ;(1+|x|) d x)$ if and only if $V \in L^{1}(\mathbb{R} ;(1+|x|) d x)$ and the scattering matrices $\tilde{S}_{(\bar{\mu}, \tilde{\sigma})}(\lambda)$ and $S(\lambda)$ in $(5.18)$ and $(5.16)$ associated with $\left(\tilde{H}_{(\bar{\mu} . \tilde{\sigma})}, H_{0}\right)$ and $\left(H, H_{0}\right)$, respectively, are related via

$$
\begin{aligned}
& \tilde{T}_{(\tilde{\mu}, \tilde{\sigma})}(\lambda)=T(\lambda), \\
& \tilde{R}_{(\tilde{\mu}, \tilde{\sigma})}^{\xi}(\lambda)=\frac{\lambda^{1 / 2} \mp i \tilde{\sigma}(-\tilde{\mu})^{1 / 2}}{\lambda^{1 / 2} \pm i \tilde{\sigma}(-\tilde{\mu})^{1 / 2}} \frac{\lambda^{1 / 2} \pm i \sigma(-\mu)^{1 / 2}}{\lambda^{1 / 2} \mp i \sigma(-\mu)^{1 / 2}} R^{r}(\lambda), \quad \lambda>0 .
\end{aligned}
$$

Proof First, we prove that $V \in L^{1}(\mathbb{R} ;(1+|x|) d x)$ if and only if $\tilde{V}_{(\tilde{\mu}, \tilde{\sigma})} \in$ $L^{1}(\mathbb{R} ;(1+|x|) d x)$ for $\mu \neq \tilde{\mu}$. We adopt the strategy of Deift and Trubowitz [8] in their proof of Theorem 3.2 (which treats the analog of Lemma 5.5 in the single commutation context; see Appendix A and especially the paragraph preceding (A.32), (A.33)). Introduce

$$
g_{\sigma}(\mu, x)=\psi_{\sigma}(\mu, x) e^{\sigma(-\mu)^{1 / 2} x}, \quad g_{-\tilde{\sigma}}(\tilde{\mu}, x)=\psi_{-\tilde{\sigma}}(\tilde{\mu}, x) e^{-\tilde{\sigma}(-\bar{\mu})^{1 / 2} x}, \quad \mu \neq \tilde{\mu}
$$

Then Lemmas 2.1 and 2.6 of [8] yield

$$
g_{\sigma}(\mu, x)=C_{\sigma . \pm}(\mu)(1+o(1)), \quad x \rightarrow \pm \infty,
$$

with $C_{\sigma, \pm}(\mu)>0$, and

$$
\begin{array}{r}
g_{\sigma}^{\prime}(\mu, x)=o(1), \quad|x| \rightarrow \infty \\
g_{\sigma}^{\prime}(\mu, \cdot) \in L^{\infty}(\mathbb{R}) \cap L^{1}(\mathbb{R} ;(1+|x|) d x)
\end{array}
$$

and similarly for $g_{-\tilde{\sigma}}(\tilde{\mu}, x)$. Next, abbreviating $W(x)=W\left(\psi_{\sigma}(\mu), \psi_{-\tilde{\sigma}}(\tilde{\mu})\right)(x)$, one computes, using (5.21),

$$
\begin{aligned}
\tilde{V}_{(\bar{\mu} ; \tilde{\sigma})}-V= & 2 W^{-2}\left[W^{\prime 2}-W W^{\prime \prime}\right] \\
= & \left\{\left[\tilde{\sigma}(-\tilde{\mu})^{1 / 2}+\sigma(-\mu)^{1 / 2}\right] g_{\sigma} g_{-\tilde{\sigma}}+g_{\sigma} g_{-\tilde{\sigma}}^{\prime}-g_{-\tilde{\sigma}} g_{\sigma}^{\prime}\right\}^{-2} \\
& \times 2(\tilde{\mu}-\mu)\left\{2 \tilde{\sigma}(-\tilde{\mu})^{1 / 2} g_{\sigma}^{2} g_{-\bar{\sigma}} g_{-\tilde{\sigma}}^{\prime}+2 \sigma(-\mu)^{1 / 2} g_{\sigma} g_{-\tilde{\sigma}}^{2} g_{\sigma}^{\prime}\right. \\
& \left.+g_{\sigma}^{2} g_{-\tilde{\sigma}}^{\prime 2}-g_{-\tilde{\sigma}}^{2} g_{\sigma}^{\prime 2}\right\}, \quad \mu \neq \tilde{\mu} .
\end{aligned}
$$


By (5.21) and (5.22), the right-hand side of (5.23) is clearly in $L^{1}(\mathbb{R} ;(1+|x|) d x)$ at least as long as $(\tilde{\mu}, \tilde{\sigma}) \neq(\mu,-\sigma)$. The case $(\tilde{\mu}, \tilde{\sigma})=(\mu,-\sigma)$ leads to a rather cumbersome $0 / 0$ expression in (5.23). Fortunately, this is quite irrelevant since we can simply apply DDM twice, that is, use the deformation sequence $(\mu, \sigma) \rightarrow$ $(\tilde{\mu},-\sigma) \rightarrow(\mu,-\sigma)$ with $\tilde{\mu} \neq \mu$ (instead of $(\mu, \sigma) \rightarrow(\mu,-\sigma)$ in one step) by appealing to Remark 4.6(ii). This then proves $\tilde{V}_{(\tilde{\mu}, \bar{\sigma})} \in L^{1}(\mathbb{R} ;(1+|x|) d x)$ if and only if $V \in L^{1}(\mathbb{R} ;(1+|x|) d x)$ in all cases.

Next, define

$$
\tilde{f}_{(\bar{\mu}, \bar{\sigma}), \pm}(\lambda, x)=\left(U_{(\bar{\mu}, \tilde{\sigma}), \pm}(\lambda) f_{ \pm}(\lambda)\right)(x), \quad \lambda>0, \tilde{\mu} \neq \mu
$$

(cf. (3.1)). Then (5.17) yields

$\tilde{f}_{(\tilde{\mu}, \tilde{\sigma}), \pm}(\lambda, x)=\frac{\lambda^{1 / 2} \pm i \tilde{\sigma}(-\tilde{\mu})^{1 / 2}}{\lambda^{1 / 2} \pm i \sigma(-\mu)^{1 / 2}}\left[e^{ \pm i \lambda^{1 / 2} x}+o(1)\right], \quad x \rightarrow \pm \infty$,

$$
\begin{aligned}
& \tilde{f}_{(\tilde{\mu}, \tilde{\sigma}), \pm}(\lambda, x)= \frac{\lambda^{1 / 2} \pm i \tilde{\sigma}(-\tilde{\mu})^{1 / 2}}{\lambda^{1 / 2} \pm i \sigma(-\mu)^{1 / 2}}\left[\frac{1}{T(\lambda)} e^{ \pm i \lambda^{1 / 2} x}+\frac{R^{\ell}(\lambda)}{T(\lambda)}\right. \\
&\left.\times \frac{\lambda^{1 / 2} \mp i \tilde{\sigma}(-\tilde{\mu})^{1 / 2}}{\lambda^{1 / 2} \pm i \tilde{\sigma}(-\tilde{\mu})^{1 / 2}} \frac{\lambda^{1 / 2} \pm i \sigma(-\mu)^{1 / 2}}{\lambda^{1 / 2} \mp i \sigma(-\mu)^{1 / 2}} e^{\mp i \lambda^{1 / 2} x}+o(1)\right], x \rightarrow \mp \infty \\
&= \frac{\lambda^{1 / 2} \pm i \tilde{\sigma}(-\tilde{\mu})^{1 / 2}}{\lambda^{1 / 2} \pm i \sigma(-\mu)^{1 / 2}}\left[\frac{1}{\tilde{T}_{(\tilde{\mu}, \tilde{\sigma})}(\lambda)} e^{ \pm i \lambda^{1 / 2} x}+\frac{\tilde{R}_{(\tilde{\mu}, \tilde{\sigma})}^{\ell}(\lambda)}{\tilde{T}_{(\tilde{\mu}, \tilde{\sigma})}(\lambda)} e^{\mp i \lambda^{1 / 2} x}+o(1)\right], \\
& x \rightarrow \mp \infty, \lambda>0, \tilde{\mu} \neq \mu,
\end{aligned}
$$

with $\tilde{T}_{(\bar{\mu}, \tilde{\sigma})}(\lambda), \tilde{R}_{(\bar{\mu}, \tilde{\sigma})}^{\ell}(\lambda)$ as given by (5.19). Applying this two-step procedure to $\tilde{S}_{(\tilde{\mu}, \bar{\sigma})}(\lambda)$ then proves (5.19) also in the remaining (sign flip) case $(\mu, \sigma) \rightarrow(\mu,-\sigma)$.

For simplicity we only considered the case $\mu, \tilde{\mu} \in\left(E_{0}, E_{1}\right)$ in Lemma 5.5. There is no problem in moving $\tilde{\mu}$ to the boundary of the interval $\left(E_{0}, E_{1}\right)$ as long as the boundary point in question is an eigenvalue of $H$ (i.e., different from $E_{1}=0$ ). Indeed, in the case $(\mu, \sigma) \rightarrow \tilde{\mu} \in\left\{E_{0}, E_{1}\right\} \cap \sigma_{d}(H), \mu \in\left(E_{0}, E_{1}\right)$, the analog of (5.19) then reads

$$
\tilde{T}_{\tilde{\mu}}(\lambda)=\frac{\lambda^{1 / 2}-i(-\tilde{\mu})^{1 / 2}}{\lambda^{1 / 2}+i(-\tilde{\mu})^{1 / 2}} T(\lambda)
$$

$$
\begin{aligned}
\tilde{R}_{\tilde{\mu}}^{\ell}(\lambda)= & \frac{\lambda^{1 / 2}-i(-\tilde{\mu})^{1 / 2}}{\lambda^{1 / 2}+i(-\tilde{\mu})^{1 / 2}} \frac{\lambda^{1 / 2} \pm i \sigma(-\tilde{\mu})^{1 / 2}}{\lambda^{1 / 2} \mp i \sigma(-\tilde{\mu})^{1 / 2}} R^{\ell}(\lambda), \\
& \lambda>0, \quad(\mu, \sigma) \in\left(E_{0}, E_{1}\right) \times\{-,+\}, \quad \tilde{\mu} \in\left\{E_{0}, E_{1}\right\} \cap \sigma_{d}(H) .
\end{aligned}
$$


One observes that the transmission coefficient in (5.19) stays invariant with respect to DDM (since Lemma 5.5 describes the isospectral case $\sigma_{(p)}\left(\tilde{H}_{(\tilde{\mu}, \tilde{\sigma})}\right)=\sigma_{(p)}(H)$ as $\left.\mu, \tilde{\mu} \in\left(E_{0}, E_{1}\right)\right)$ whereas (5.25) exhibits a change of $T(\lambda)$ (as it must since now $\tilde{\mu} \in\left\{E_{0}, E_{1}\right\} \cap \sigma_{d}(H)$ got knocked out of the spectrum of $\tilde{H}_{(\tilde{\mu}, \tilde{\sigma})}, \sigma_{(p)}\left(\tilde{H}_{(\tilde{\mu}, \tilde{\sigma})}\right)=$ $\left.\sigma_{(p)}(H) \backslash\{\tilde{\mu}\}\right)$. In this context we invite the reader to compare with the corresponding single and double commutation results in Appendices A and B (see (A.32), (A.33), and (B.35), (B.36)).

Remark 5.6 It should be pointed out at the end that the material in this paper is not at all confined to Schrödinger or Sturm-Liouville operators. In fact, (a generalization of) Lemma 2.2 for general second-order finite-difference (Jacobi) operators appeared in [52]. Moreover, the discrete analog of DDM was used in [28] to construct an explicit realization of the isospectral torus for algebro-geometric quasi-periodic Jacobi operators. A detailed analysis of the discrete version of DDM will be given in [53].

\section{ACKNOWLEDGEMENT}

F. G. is indebted to C. W. Peck and A. Kechris for kind invitations to Caltech during the summers of 1994 and 1996 where some of this work was done. The extraordinary hospitality and support by the Department of Mathematics at Caltech are gratefully acknowledged.

\section{Appendix A. The single commutation or Crum-Darboux method}

We briefly summarize the main spectral characteristics of the single commutation method (abbreviated occasionally as SCM in this appendix). The principal source for the following material is the fundamental paper by Deift [7] (see also [8], [26], [50]). Further hints to the literature and to applications of this method in spectral theory and completely integrable systems are collected at the end of this appendix.

Suppose

$$
V \in L_{\text {loc }}^{1}(\mathbb{R}) \text { is real-valued }
$$

and assume that the differential expression

$$
\tau=-\frac{d^{2}}{d x^{2}}+V(x), \quad x \in \mathbb{R} \quad \text { is non-oscillatory at both } \pm \infty
$$

Denote by $H$ the uniquely associated self-adjoint operator in $L^{2}(\mathbb{R})$, maximally defined, that is,

$$
\begin{aligned}
H f & =\tau f \\
f \in \mathcal{D}(H) & =\left\{g \in L^{2}(\mathbb{R}) \mid g, g^{\prime} \in A C_{\mathrm{loc}}(\mathbb{R}) ; \tau g \in L^{2}(\mathbb{R})\right\} .
\end{aligned}
$$


Because of (A.2), $H$ is bounded from below,

$$
H \geq \Sigma_{0}, \quad \Sigma_{0}=\inf \sigma(H)>-\infty
$$

Next, let $\psi_{ \pm}(z, x), z \in \mathbb{C} \backslash \sigma_{\text {ess }}(H)$ be the unique (up to constant multiples) solutions of

$$
\tau \psi(z)=z \psi(z)
$$

satisfying for all $z \in \mathbb{C} \backslash \sigma_{\text {ess }}(H), R \in \mathbb{R}$,

$$
\psi_{ \pm}(z,, \cdot) \in L^{2}((R, \pm \infty)) \quad \text { and } \psi_{ \pm}(\lambda, x)>0 \quad \text { for } \lambda<\Sigma_{0}
$$

(The latter condition in (A.6) can always be achieved since $\psi_{ \pm}(\lambda, x) \neq 0$ for $\lambda<\Sigma_{0}$.) One defines

(A.7) $\psi_{\nu_{1}}\left(\lambda_{1}, x\right)=\frac{1}{2}\left(1-\nu_{1}\right) \psi_{-}\left(\lambda_{1}, x\right)+\frac{1}{2}\left(1+\nu_{1}\right) \psi_{+}\left(\lambda_{1}, x\right), \quad \nu_{1} \in[-1,1], \lambda_{1}<\Sigma_{0}$

(we identify $\psi_{ \pm 1}=\psi_{ \pm}$for notational convenience) and

$$
\begin{aligned}
\phi_{\nu_{1}}\left(\lambda_{1}, x\right) & =\psi_{\nu_{1}}^{\prime}\left(\lambda_{1}, x\right) / \psi_{\nu_{1}}\left(\lambda_{1}, x\right), \\
\alpha_{\nu_{1}}\left(\lambda_{1}\right) & =\frac{d}{d x}+\phi_{\nu_{1}}\left(\lambda_{1}, x\right), \quad \alpha_{\nu_{1}}\left(\lambda_{1}\right)^{+}=-\frac{d}{d x}+\phi_{\nu_{1}}\left(\lambda_{1}, x\right) .
\end{aligned}
$$

(We note that $\psi_{\nu_{1}}\left(\lambda_{1}, x\right) \neq 0$ for $\lambda_{1}<\Sigma_{0}$.) One infers that

$$
\tau=\alpha_{\nu_{1}}\left(\lambda_{1}\right) \alpha_{\nu_{1}}\left(\lambda_{1}\right)^{+}+\lambda_{1}=-\frac{d^{2}}{d x^{2}}+V(x)
$$

is independent of $\nu_{1} \in[-1,1]$ and $\lambda_{1}<\Sigma_{0}$ and introduces a commutation of $\alpha_{\nu_{1}}\left(\lambda_{1}\right)$ and $\alpha_{\nu_{1}}\left(\lambda_{1}\right)^{+}$,

$$
\begin{aligned}
\tilde{\tau}_{\nu_{1}}\left(\lambda_{1}\right) & =\alpha_{\nu_{1}}\left(\lambda_{1}\right)^{+} \alpha_{\nu_{1}}\left(\lambda_{1}\right)+\lambda_{1}=-\frac{d^{2}}{d x^{2}}+\tilde{V}_{\nu_{1}}\left(\lambda_{1}, x\right), \quad x \in \mathbb{R}, \\
\tilde{V}_{\nu_{1}}\left(\lambda_{1}, x\right) & =V(x)-2\left\{\ln \left[\psi_{\nu_{1}}\left(\lambda_{1}, x\right)\right]\right\}^{\prime \prime}, \quad \lambda_{1}<\Sigma_{0}, \nu_{1} \in[-1,1] .
\end{aligned}
$$

One verifies

$$
\begin{gathered}
\alpha_{\nu_{1}}\left(\lambda_{1}\right)^{+} \psi_{\nu_{1}}\left(\lambda_{1}\right)=0, \quad \alpha_{\nu_{1}}\left(\lambda_{1}\right) \psi_{\nu_{1}}\left(\lambda_{1}\right)^{-1}=0, \\
\psi_{ \pm}\left(\lambda_{1}, \cdot\right) \in L^{2}((R, \pm \infty)), \quad \psi_{ \pm}\left(\lambda_{1}, \cdot\right) \notin L^{2}((R, \mp \infty)), \quad R \in \mathbb{R}, \\
\psi_{\nu_{1}}\left(\lambda_{1}, \cdot\right) \notin L^{2}((-\infty, R)) \cup L^{2}((R, \infty)), \quad R \in \mathbb{R} \\
\psi_{\nu_{1}}\left(\lambda_{1}, \cdot \cdot\right)^{-1} \in L^{2}(\mathbb{R}), \quad \nu_{1} \in(-1,1) .
\end{gathered}
$$


Next, let $\overline{A_{\nu_{1}}\left(\lambda_{1}\right)}, \overline{A_{\nu_{1}}\left(\lambda_{1}\right)} *$, and $\tilde{H}_{\nu_{1}}\left(\lambda_{1}\right)$ be the following closed linear operators in $L^{2}(\mathbb{R})$ associated with $\alpha_{\nu_{1}}\left(\lambda_{1}\right), \alpha_{\nu_{1}}\left(\lambda_{1}\right)^{+}$, and $\tilde{\tau}_{\nu_{1}}\left(\lambda_{1}\right)$, respectively. Consider

$$
\mathcal{D}_{0}=\{g \in \mathcal{D}(H) \mid \operatorname{supp}(g) \text { compact }\}
$$

$$
A_{\nu_{1}}\left(\lambda_{1}\right)=\left.\alpha_{\nu_{1}}\left(\lambda_{1}\right)\right|_{\mathcal{D}_{0}}
$$

Then,

$$
\left.A_{\nu_{1}}\left(\lambda_{1}\right)^{*}\right|_{\mathcal{D}_{0}}=\left.\alpha_{\nu_{1}}\left(\lambda_{1}\right)^{+}\right|_{\mathcal{D}_{0}}
$$

and (cf. [30])

$$
H=\overline{A_{\nu_{1}}\left(\lambda_{1}\right)} \overline{A_{\nu_{1}}\left(\lambda_{1}\right)}{ }^{*}+\lambda_{1}, \quad \tilde{H}_{\nu_{1}}\left(\lambda_{1}\right)=\overline{A_{\nu_{1}}\left(\lambda_{1}\right)} \bar{A}_{\nu_{1}\left(\lambda_{1}\right)}+\lambda_{1} .
$$

In particular, by Lemma 5.1(i), $\tilde{\tau}_{\nu_{1}}\left(\lambda_{1}\right)$ (and, of course, $\tau$ ) is l.p. at $\pm \infty$ since $\tilde{H}_{\nu_{1}}\left(\lambda_{1}\right) \geq \lambda_{1}\left(H \geq \Sigma_{0}\right)$. Let

$$
\begin{gathered}
{\overline{A_{\nu_{1}}\left(\lambda_{1}\right)}}^{*}=S_{\nu_{1}}\left(\lambda_{1}\right)\left|\overline{A_{\nu_{1}}\left(\lambda_{1}\right)^{*}}\right|=\left|\overline{A_{\nu_{1}}\left(\lambda_{1}\right)}\right| S_{\nu_{1}}\left(\lambda_{1}\right), \\
\left.\mid \overline{A_{\nu_{1}}\left(\lambda_{1}\right)}\right)^{*}\left|=\left[\overline{A_{\nu_{1}}\left(\lambda_{1}\right)} \overline{A_{\nu_{1}}\left(\lambda_{1}\right)^{*}}\right]^{1 / 2}, \quad\right| \overline{A_{\nu_{1}}\left(\lambda_{1}\right)} \mid=\left[\overline{A_{\nu_{1}}\left(\lambda_{1}\right)} \bar{A}_{\nu_{1}}\left(\lambda_{1}\right)\right]^{1 / 2}
\end{gathered}
$$

denote the polar decomposition of ${\overline{A_{\nu_{1}}\left(\lambda_{1}\right)}}^{*}$, where

$$
S_{\nu_{1}}\left(\lambda_{1}\right): L^{2}(\mathbb{R}) \rightarrow \operatorname{Ker}\left(\overline{A_{\nu_{1}}\left(\lambda_{1}\right)}\right)^{\dot{1}}
$$

is unitary. At this point we used the fact that $\operatorname{Ker}\left({\overline{A_{\nu_{1}}\left(\lambda_{1}\right)}}^{*}\right)=\{0\}$ by the hypothesis $\lambda_{1}<\Sigma_{0}=\inf \sigma(H)$. Moreover, we introduce the orthogonal projection $\tilde{P}_{\nu_{1}}\left(\lambda_{1}\right)$ onto $\operatorname{Ker}\left(\overline{A_{\nu_{1}}\left(\lambda_{1}\right)}\right)$, that is,

$$
\tilde{P}_{\nu_{1}}\left(\lambda_{1}\right)= \begin{cases}0, & \nu_{1} \in\{-1,1\} \\ \left\|\psi_{\nu_{1}}\left(\lambda_{1}\right)^{-1}\right\|_{2}^{-2}\left(\psi_{\nu_{1}}\left(\lambda_{1}\right)^{-1}, \cdot\right) \psi_{\nu_{1}}\left(\lambda_{1}\right)^{-1}, & \nu_{1} \in(-1,1)\end{cases}
$$

(cf. (A.12)).

The fundamental result regarding the spectra of $H$ and $\tilde{H}_{\nu_{1}}\left(\lambda_{1}\right)$ then follows as a special case of the unitary equivalence of $\bar{A} \bar{A}^{*}$ and $\bar{A}^{*} \bar{A}$, restricted to the orthogonal complements of their respective null spaces (see Deift [7]).

Theorem A.1 (Deift [7], see also [26], [50]) Let $H, \tilde{H}_{\nu_{1}}\left(\lambda_{1}\right), \nu_{1} \in[-1,1]$, $\lambda_{1}<\Sigma_{0}=\inf \sigma(H)$, and $S_{\nu_{1}}\left(\lambda_{1}\right), \tilde{P}_{\nu_{1}}\left(\lambda_{1}\right)$ be given as in (A.3), (A.16), and (A.18), (A.19). Then

$$
\tilde{H}_{\nu_{1}}\left(\lambda_{1}\right) \mid \operatorname{Ran}\left(1-\tilde{P}_{\nu_{1}}\left(\lambda_{1}\right)\right)=S_{\nu_{1}}\left(\lambda_{1}\right) H S_{\nu_{1}}\left(\lambda_{1}\right)^{-1},
$$


that is, $\tilde{H}_{ \pm 1}\left(\lambda_{1}\right)$ and $H$, and $\tilde{H}_{\nu_{1}}\left(\lambda_{1}\right)$ and $H, \nu_{1} \in(-1,1)$, restricted to the orthogonal complement of the (one-dimensional) eigenspace associated with the eigenvalue $\lambda_{1}$ of $\tilde{H}_{\nu_{1}}\left(\lambda_{1}\right)$, are unitarily equivalent. In particular,

$$
\begin{aligned}
\sigma_{(p)}\left(\tilde{H}_{\nu_{1}}\left(\lambda_{1}\right)\right) & = \begin{cases}\sigma_{(p)}(H), & \nu_{1} \in\{-1,1\}, \\
\sigma_{(p)}(H) \cup\left\{\lambda_{1}\right\}, & \nu_{1} \in(-1,1),\end{cases} \\
\operatorname{Ker}\left(\tilde{H}_{\nu_{1}}\left(\lambda_{1}\right)-\lambda_{1}\right) & = \begin{cases}\{0\}, & \nu_{1} \in\{-1,1\}, \\
\operatorname{span}\left\{\psi_{\nu_{1}}\left(\lambda_{1}\right)^{-1}\right\}, & \nu_{1} \in(-1,1),\end{cases} \\
\sigma_{\text {ess }, \mathbf{a c}, \mathbf{s c}}\left(\tilde{H}_{\nu_{1}}\left(\lambda_{1}\right)\right) & =\sigma_{\text {ess,ac,sc }}(H) .
\end{aligned}
$$

Next, we describe a variety of additional results and possible extensions paralleling Section 5. This is intended to facilitate comparisons with DDM as well as the double commutation method in Appendix B.

One verifies that

$$
\tilde{\psi}_{\nu_{1}, \pm}\left(z, \lambda_{1}, x\right)=W\left(\psi_{ \pm}(z), \psi_{\nu_{1}}\left(\lambda_{1}\right)\right)(x) / \psi_{\nu_{1}}\left(\lambda_{1}, x\right)
$$

satisfies

$$
\begin{gathered}
\tilde{\tau}_{\nu_{1}}\left(\lambda_{1}\right) \tilde{\psi}_{\nu_{1}, \pm}\left(z, \lambda_{1}\right)=z \tilde{\psi}_{\nu_{1}, \pm}\left(z, \lambda_{1}\right) \\
\tilde{\psi}_{\nu_{1}, \pm}\left(z, \lambda_{\mathrm{J}}, \cdot\right) \in L^{2}((R, \pm \infty)), \quad z \in \mathbb{C} \backslash \sigma(H), R \in \mathbb{R}
\end{gathered}
$$

(The latter fact is proven in [26], Appendix A for $z \leq \lambda_{1}$; one can use (2.16) to extend it to $z \in \mathbb{C} \backslash \sigma(H)$.) Hence, normalizing $\psi_{ \pm}(z, x)$ temporarily (and without loss of generality) by

$$
\psi_{ \pm}\left(\lambda_{1}, x_{0}\right)=1, \quad x_{0} \in \mathbb{R} \text { fixed }
$$

the Weyl-Titchmarsh $m$-function $\tilde{m}_{\nu_{1} \pm \pm}\left(z, \lambda_{1}\right)$ associated with $\tilde{H}_{\nu_{1}}\left(\lambda_{1}\right)$ and the reference point $x_{0}$ is given by (cf. [21])

$$
\begin{gathered}
\tilde{m}_{\nu_{1}, \pm}\left(z, \lambda_{1}\right)=\frac{z-\lambda_{1}}{\cot \left(\alpha_{\nu_{1}}\left(\lambda_{1}\right)\right)-m_{ \pm}(z)}-\cot \left(\alpha_{\nu_{1}}\left(\lambda_{1}\right)\right), \\
\cot \left(\alpha_{\nu_{1}}\left(\lambda_{1}\right)\right)=\frac{1}{2}\left(1-\nu_{1}\right) m_{-}\left(\lambda_{1}\right)+\frac{1}{2}\left(1+\nu_{1}\right) m_{+}\left(\lambda_{1}\right), \quad \nu_{1} \in[-1,1], z \in \mathbb{C} \backslash \mathbb{R} .
\end{gathered}
$$

Here use has been made of

$$
\tilde{m}_{\nu_{1}, \pm}\left(z, \lambda_{1}\right)=\tilde{\psi}_{\nu_{1}, \pm}^{\prime}\left(z, \lambda_{1}, x_{0}\right)
$$

(A.22), (A.24), and the fact that $\tilde{\tau}_{\nu_{1}}\left(\lambda_{1}\right)$ is 1.p. at $\pm \infty$. 
Given (A.25) one can compute $\tilde{M}_{\nu_{1}}\left(z, \lambda_{1}\right)$, the Weyl-Titchmarsh $M$-matrix in $\mathbb{C}^{2}$ associated with $\tilde{H}_{\nu_{1}}\left(\lambda_{1}\right)$ in terms of $M(z)$, the $M$-matrix of $H$ (see (4.1)). One obtains

$$
\begin{gathered}
\tilde{M}_{\nu_{1}, 1,1}\left(z, \lambda_{1}\right)=\frac{\cot ^{2}\left(\alpha_{\nu_{1}}\left(\lambda_{1}\right)\right)}{z-\lambda_{1}} M_{1,1}(z)+2 \cot \left(\alpha_{\nu_{1}}\left(\lambda_{1}\right)\right)\left[1-\frac{\cot ^{2}\left(\alpha_{\nu_{1}}\left(\lambda_{1}\right)\right)}{z-\lambda_{1}}\right] M_{1,2}(z) \\
+\left[\left(z-\lambda_{1}\right)-2 \cot ^{2}\left(\alpha_{\nu_{1}}\left(\lambda_{1}\right)\right)+\frac{\cot ^{4}\left(\alpha_{\nu_{1}}\left(\lambda_{1}\right)\right)}{z-\lambda_{1}}\right] M_{2,2}(z)
\end{gathered}
$$

$$
\tilde{M}_{\nu_{1}, 1,2}\left(z, \lambda_{1}\right)=-\frac{\cot \left(\alpha_{\nu_{1}}\left(\lambda_{1}\right)\right)}{z-\lambda_{1}} M_{1,1}(z)+\left[\frac{2 \cot ^{2}\left(\alpha_{\nu_{1}}\left(\lambda_{1}\right)\right)}{z-\lambda_{1}}-1\right] M_{1,2}(z)
$$

$$
\begin{array}{r}
\tilde{M}_{\nu_{1}, 2,2}\left(z, \lambda_{1}\right)=\frac{1}{z-\lambda} M_{1,1}(z)-\frac{2 \cot \left(\alpha_{\nu_{1}}\left(\lambda_{1}\right)\right)}{z-\lambda_{1}} M_{1,2}(z)+\frac{\cot ^{2}\left(\alpha_{\nu_{1}}\left(\lambda_{1}\right)\right)}{z-\lambda_{1}} M_{2,2}(z), \\
\quad z \in \mathbb{C} \backslash \mathbb{R} .
\end{array}
$$

One readily confirms that all matrix elements $\tilde{M}_{\nu_{1}, p, q}\left(z, \lambda_{1}\right), 1 \leq p, q \leq 2$ have a pole at $z=\lambda_{1}$ if and only if $\nu_{1} \in(-1,1)$ (i.e., if and only if $\left.\cot \left(\alpha_{\nu_{1}}\left(\lambda_{1}\right)\right) \neq m_{ \pm}\left(\lambda_{1}\right)\right)$ in agreement with Theorem A.1. Moreover, we might note that $m_{-}\left(\lambda_{1}\right) \neq m_{+}\left(\lambda_{1}\right)$ since $\lambda_{1}<\Sigma_{0}=\inf \sigma(H)$.

One could use (A.26)-(A.28) to compute the corresponding $\mathbb{C}^{2}$-valued spectral matrix $\tilde{\rho}_{\nu_{1}}\left(\lambda, \lambda_{1}\right)$ of $\tilde{H}_{\nu_{1}}\left(\lambda_{1}\right)$ in terms of $\rho(\lambda)$, the one associated with $H$. The resulting formulas (although providing an alternative proof of Theorem A.1), however, are rather complex and hence omitted. (A.26)-(A.28) become simpler if the Dirichlet boundary condition $\psi\left(x_{0} \pm 0\right)=0$, used to compute $\tilde{m}_{\nu_{1}, \pm}\left(z, \lambda_{1}\right)$, $\tilde{M}_{\nu_{1}}\left(z, \lambda_{1}\right)$, is replaced by $\sin \left(\alpha_{\nu_{1}}\left(\lambda_{1}\right)\right) \psi^{\prime}\left(x_{0} \pm 0\right)+\cos \left(\alpha_{\nu_{1}}\left(\lambda_{1}\right)\right) \psi\left(x_{0} \pm 0\right)=0$. We will not pursue this now but return to this approach in Appendix $B$.

Iterations of SCM can be handled as follows. Assume $V \in L_{\text {loc }}^{l}(\mathbb{R})$ to be realvalued and pick $\lambda_{1}<\lambda_{2}<\cdots<\lambda_{N}<\Sigma_{0}=\inf \sigma(H), \nu_{j} \in[-1,1], C_{ \pm, j}>0$, $1 \leq j \leq N, N \in \mathbb{N}$. Then the SCM result after $N$ iteration steps, denoted by $\tilde{\tau}_{\nu_{1}, \ldots, \nu_{N}}\left(\lambda_{1}, \ldots, \lambda_{N}\right)$, reads as follows:

$$
\begin{gathered}
\tilde{\tau}_{\nu_{1}, \ldots, \nu_{N}}\left(\lambda_{1}, \ldots, \lambda_{N}\right)=-\frac{d^{2}}{d x^{2}}+\tilde{V}_{\nu_{1}, \ldots, \nu_{N}}\left(\lambda_{1}, \ldots, \lambda_{N}, x\right), \quad x \in \mathbb{R}, \\
\tilde{V}_{\nu_{1}, \ldots, \nu_{N}}\left(\lambda_{1}, \ldots, \lambda_{N}, x\right)=V(x)-2\left\{\ln \left[W\left(\psi_{\nu_{1}}\left(\lambda_{1}\right), \ldots, \psi_{\nu_{N}}\left(\lambda_{N}\right)\right)(x)\right]\right\}^{\prime \prime}, \\
\psi_{\nu_{j}}\left(\lambda_{j}, x\right)=\frac{1}{2}\left(1-\nu_{j}\right) C_{-j} \psi_{-}\left(\lambda_{j}, x\right)+\frac{1}{2}\left(1+\nu_{j}\right) C_{+j} \psi_{+}\left(\lambda_{j}, x\right), \quad 1 \leq j \leq N .
\end{gathered}
$$


The obvious analog of Theorem A.1 (distinguishing between $\nu_{j} \in(-1,1)$ and $\left.\nu_{j} \in\{-1,1\}\right)$ then applies to $\tilde{H}_{\nu_{1}, \ldots, \nu_{N}}\left(\lambda_{1}, \ldots, \lambda_{N}\right)$, the unique semi-bounded selfadjoint operator associated with $\tilde{\tau}_{\nu_{1}, \ldots, \nu_{N}}\left(\lambda_{1}, \ldots, \lambda_{N}\right)$ (see [26], Appendix A for more details).

In analogy to Lemma 5.4, one infers from (A.25) that $\tilde{H}_{\nu_{1}}\left(\lambda_{1}\right)$ is reflectionless if and only if $H$ is (as observed in [21]).

In order to obtain the Sturm-Liouville generalization of (A.1)-(A.16) (see [27], [50]), one assumes

$$
\begin{array}{r}
p, p^{\prime}, k, k^{\prime} \in A C_{\mathrm{loc}}\left((a, b), \quad q \in L_{\mathrm{loc}}^{1}((a, b))\right. \text { real-valued, } \\
p>0, k>0,-\infty \leq a<b \leq \infty
\end{array}
$$

and makes the changes

$$
\begin{aligned}
& \alpha_{\nu_{1}}\left(\lambda_{1}\right) \rightarrow \hat{\alpha}_{\nu_{1}}\left(\lambda_{1}\right)=\frac{1}{k(x)}\left(\sqrt{k(x) p(x)} \frac{d}{d x}+\phi_{\nu_{1}}\left(\lambda_{1}, x\right)\right) \\
& \alpha_{\nu_{1}}\left(\lambda_{1}\right)^{+} \rightarrow \widehat{\alpha}_{\nu_{1}}\left(\lambda_{1}\right)^{+}=\frac{1}{k(x)}\left(-\frac{d}{d x} \sqrt{k(x) p(x)}+\phi_{\nu_{1}}\left(\lambda_{1}, x\right)\right), \\
& \tau \rightarrow \widehat{\tau}=\hat{\alpha}_{\nu_{1}}\left(\lambda_{1}\right) \hat{\alpha}_{\nu_{1}}\left(\lambda_{1}\right)^{+}+\lambda_{1}=\frac{1}{k(x)}\left(-\frac{d}{d x} p(x) \frac{d}{d x}+q(x)\right), \\
& \tilde{\tau}_{\nu_{1}}\left(\lambda_{1}\right) \rightarrow \widetilde{\widehat{\tau}}_{\nu_{1}}\left(\lambda_{1}\right)=\widehat{\alpha}_{\nu_{1}}\left(\lambda_{1}\right)^{-} \widehat{\alpha}_{\nu_{1}}\left(\lambda_{1}\right)+\lambda_{1}=\frac{1}{k(x)}\left(-\frac{d}{d x} p(x) \frac{d}{d x}+\tilde{q}_{\nu_{1}}\left(\lambda_{1}, x\right)\right), \\
& \tilde{q}_{\nu_{\mathrm{l}}}\left(\lambda_{1}, x\right)=q(x)-\frac{p^{\prime \prime}(x)}{2}+\frac{p^{\prime}(x)^{2}}{4 p(x)}+\frac{3 k^{\prime}(x)^{2} p(x)}{4 k(x)^{2}}-\frac{k^{\prime \prime}(x) p(x)}{2 k(x)} \\
& +\left(\frac{1}{k(x)}(k(x) p(x))^{\prime}-2 \frac{d}{d x} p(x)\right) \frac{d}{d x} \ln \left[\psi_{\nu_{1}}\left(\lambda_{1}, x\right)\right],
\end{aligned}
$$

$\widehat{\tau} \psi_{\nu_{1}}\left(\lambda_{1}\right)=\lambda_{1} \psi_{\nu_{1}}\left(\lambda_{1}\right), \quad \phi_{\nu_{1}}\left(\lambda_{1}, x\right)=\left(\sqrt{k(x) p(x)} \psi_{\nu_{1}}\left(\lambda_{1}, x\right)\right)^{\prime} / \psi_{\nu_{1}}\left(\lambda_{1}, x\right)$.

It remains to sketch the scattering theory formulas analogous to (5.19), assuming $V \in L^{1}(\mathbb{R} ;(1+|x|) d x)$ to be real-valued. (We use the conventions established in (5.11)-(5.17).) It was proved by Deift and Trubowitz ([8], Theorem 3.2) that $\tilde{V}_{\nu_{1}}\left(\lambda_{1}\right) \in L^{1}\left(\mathbb{R}_{;}(1+|x|) d x\right)$ if and only if $V$ is, and also the scattering matrix $\tilde{S}_{\nu_{1}}\left(\lambda, \lambda_{1}\right)$ associated with the pair $\left(\tilde{H}_{\nu_{1}}\left(\lambda_{1}\right), H_{0}\right)$ in terms of $S(\lambda)$ in (5.16), the one corresponding to $\left(H, H_{0}\right)$, was determined as follows:

$$
\begin{aligned}
& \tilde{T}_{\nu_{1}}\left(\lambda, \lambda_{1}\right)=\frac{\lambda^{1 / 2}+i\left(-\lambda_{1}\right)^{1 / 2}}{\lambda^{1 / 2}-i\left(-\lambda_{1}\right)^{1 / 2}} T(\lambda), \\
& \tilde{R}_{\nu_{1}}^{\ell}\left(\lambda, \lambda_{1}\right)=-\frac{\lambda^{1 / 2}+i\left(-\lambda_{1}\right)^{1 / 2}}{\lambda^{1 / 2}-i\left(-\lambda_{1}\right)^{1 / 2}} R^{\ell}(\lambda), \quad \lambda>0, \lambda_{1}<\Sigma_{0}, \nu_{1} \in(-1,1),
\end{aligned}
$$


(A.33)

$$
\begin{aligned}
& \tilde{T}_{\nu_{1}}\left(\lambda, \lambda_{1}\right)=T(\lambda), \\
& \tilde{R}_{\nu_{1}}^{r}\left(\lambda, \lambda_{1}\right)=-\frac{\lambda^{1 / 2} \pm i \nu_{1}\left(-\lambda_{1}\right)^{1 / 2}}{\lambda^{1 / 2} \mp i \nu_{1}\left(-\lambda_{1}\right)^{1 / 2}} R^{\dot{r}}(\lambda), \quad \lambda>0, \lambda_{1}<\Sigma_{0}, \nu_{1} \in\{-1,1\} .
\end{aligned}
$$

Further generalizations of (A.32), (A.33) in the context of supersymmetric quantum mechanics can be found in [3] and [22].

The discrete analog of SCM for general second-order finite-difference (Jacobi) operators has been developed in detail in [28].

Finally, we provide a brief historical account and hints to some applications of SCM. SCM, or as it is often called, the Crum-Darboux method, (A.7)-(A.11) goes back at least to Jacobi [33] and Darboux [6]. Important later contributions are due to Crum [5], Schmincke [50], and especially, Deift [7] (see also [8]). In particular, the spectral deformation results of this method, as summarized in Theorem A.1, are due to Deift [7].

In recent years, this method has been applied to a description of the isospectral manifold of periodic and algebro-geometric quasi-periodic finite-gap solutions of the (m)KdV hierarchy (see, e.g, [10], [11], [17], [18], [22], [29], [40], [41], [42], [43], [44], and the references therein) and the construction of soliton solutions (resp., reflectionless potentials) of the $(\mathrm{m}) \mathrm{KdV}$ hierarchy relative to given background (base) solutions (resp., potentials) by means of Bäcklund transformations (cf., e.g., [7], [8], [14], [17], [22], [26], [42], and the literature cited therein).

As is obvious from (A.11), $\psi_{\nu_{1}}\left(\lambda_{1}, x\right)$ had to be chosen zero-free (and hence $\left.\lambda_{1}<\Sigma_{0}\right)$ in order to guarantee $V_{\nu_{1}}\left(\lambda_{1}\right) \in L_{\text {loc }}(\mathbb{R})$. This considerable restriction on the range of $\lambda_{1}$ will be overcome in the following appendix.

\section{Appendix B. The double commutation method}

We review the double commutation method (occasionally abbreviated as DCM) to insert eigenvalues into spectral gaps of general background (base) Schrödinger operators following [19] and [27]. Applications of this method and pertinent references to the literature are collected at the end of this appendix.

Assuming $V$ satisfies

$$
V \in L_{\text {loc }}^{l}(\mathbb{R}), \quad V \text { real-valued }
$$

and introducing the differential expression $\tau=-d^{2} / d x^{2}+V(x), x \in \mathbb{R}$, we pick $\lambda_{0} \in \mathbb{R}$ and $\eta_{ \pm}\left(\lambda_{0}, x\right)$ satisfying

$$
\begin{gathered}
\tau \psi\left(\lambda_{0}\right)=\lambda_{0} \psi\left(\lambda_{0}\right) \\
\eta_{ \pm}\left(\lambda_{0}, \cdot\right) \in L^{2}((R, \pm \infty)), \quad R \in \mathbb{R}, \eta_{ \pm}\left(\lambda_{0}, x\right) \text { real-valued. }
\end{gathered}
$$


Given $\eta_{ \pm}\left(\lambda_{0}, x\right)$, we define the self-adjoint background (base) operator $H_{ \pm}$in $L^{2}(\mathbb{R})$ via

$$
\begin{aligned}
H_{ \pm} f= & \tau f \\
f \in \mathcal{D}\left(H_{ \pm}\right)= & \left\{g \in L^{2}(\mathbb{R}) \mid g, g^{\prime} \in A C_{\mathrm{loc}}(\mathbb{R}) ; \tau g \in L^{2}(\mathbb{R})\right. \\
& \left.\lim _{x \rightarrow \omega \infty} W\left(\eta_{ \pm}\left(\lambda_{0}\right), g\right)(x)=0 \text { if } \tau \text { is l.c. at } \omega \infty, \omega \in\{-,+\}\right\} .
\end{aligned}
$$

Our choice of notation purposely stresses a possible dependence of $H_{ \pm}$on $\eta_{ \pm}\left(\lambda_{0}, x\right)$. If $\tau$ is in the 1.p. case at $\omega \infty, \omega \in\{-,+\}$, the corresponding boundary condition in (B.3) is superfluous at $\omega \infty$ and hence to be deleted from (B.3). In particular, if $\tau$ is l.p. at $\pm \infty$, then $H_{-}=H_{+}=H$ is independent of the choice of $\eta_{ \pm}\left(\lambda_{0}, x\right)$.

Next, denote

$$
L_{\mathrm{loc}}^{2}([\mp \infty, \pm \infty))=\left\{g \in L_{\mathrm{loc}}^{2}(\mathbb{R}) \mid g \in L^{2}((\mp \infty, c))\right\}
$$

for some $c \in \mathbb{R}$ and pick $\gamma_{1} \in(0, \infty), \lambda_{\mathbf{l}} \in \mathbb{R}$ and $\psi_{ \pm}(z, x)$ satisfying

$$
\begin{gathered}
\tau \psi(z)=z \psi(z), \\
\psi_{ \pm}(z, \cdot) \in L^{2}((R, \pm \infty)), \quad z \in \mathbb{C} \backslash \sigma_{\text {ess }}\left(H_{ \pm}\right), R \in \mathbb{R},
\end{gathered}
$$

$$
\begin{gathered}
\psi_{ \pm}(\lambda, x) \quad \text { real-valued for } \lambda \in \mathbb{R} \\
\psi_{ \pm}\left(\lambda_{1}, \cdot\right) \in L^{2}((R, \pm \infty)), \quad R \in \mathbb{R} \\
\lim _{x \rightarrow \omega \infty} W\left(\eta_{ \pm}\left(\lambda_{0}\right), \psi_{ \pm}\left(\lambda_{1}\right)\right)(x)=0 \quad \text { if } \tau \text { is l.c. at } \omega \infty, \omega \in\{-,+\} .
\end{gathered}
$$

As in (B.3), the last condition in (B.4) is superfluous at $\omega \infty$ if $\tau$ is l.p. at $\omega \infty$, $\omega \in\{-,+\}$. Given $\gamma_{1}>0$ and $\psi_{ \pm}\left(\lambda_{1}, x\right)$, we define the linear transformation

$$
\widehat{U}_{ \pm, \gamma_{1}}\left(\lambda_{1}\right):\left\{\begin{array}{l}
L_{\mathrm{loc}}^{2}([ \pm \infty, \mp \infty)) \rightarrow L_{\mathrm{loc}}^{2}([ \pm \infty, \mp \infty)) \\
f(x) \rightarrow \tilde{f}_{ \pm, \gamma_{1}}\left(\lambda_{1}, x\right)=f(x) \pm \gamma_{1} \tilde{\psi}_{ \pm, \gamma_{1}}\left(\lambda_{1}, x\right) \int_{ \pm \infty}^{x} d x^{\prime} \psi_{ \pm}\left(\lambda_{1}, x^{\prime}\right) f\left(x^{\prime}\right)
\end{array}\right.
$$

where

(B.6)

$$
\tilde{\psi}_{ \pm, \gamma_{1}}\left(\lambda_{1}, x\right)=\left(\hat{U}_{ \pm, \gamma_{1}}\left(\lambda_{1}\right) \psi_{ \pm}\left(\lambda_{1}\right)\right)(x)=\left[1 \mp \gamma_{1} \int_{ \pm \infty}^{x} d x^{\prime} \psi_{ \pm}\left(\lambda_{1}, x^{\prime}\right)^{2}\right]^{-1} \psi_{ \pm}\left(\lambda_{1}, x\right)
$$

By inspection, one infers for the inverse transformation

$$
\widehat{U}_{ \pm, \gamma_{1}}\left(\lambda_{1}\right)^{-1}:\left\{\begin{array}{l}
L_{\mathrm{loc}}^{2}([ \pm \infty, \mp \infty)), \rightarrow L_{\mathrm{loc}}^{2}([ \pm \infty, \mp \infty)) \\
h(x) \rightarrow h(x) \mp \gamma_{1} \psi_{ \pm}\left(\lambda_{1}, x\right) \int_{ \pm \infty}^{x} d x^{\prime} \tilde{\psi}_{ \pm, \gamma_{1}}\left(\lambda_{1}, x^{\prime}\right) h\left(x^{\prime}\right)
\end{array}\right.
$$


We list a few more facts (cf. [27]) which explain Lemma B.1 and Theorem B.2 below.

$$
1 \pm \gamma_{1} \int_{ \pm \infty}^{x} d x^{\prime} \tilde{\psi}_{ \pm, \gamma_{1}}\left(\lambda_{1}, x^{\prime}\right)^{2}=\left[1 \mp \gamma_{1} \int_{ \pm \infty}^{x} d x^{\prime} \psi_{ \pm}\left(\lambda_{1}, x^{\prime}\right)^{2}\right]^{-1}
$$

$$
\tilde{\psi}_{ \pm, \gamma_{1}}\left(\lambda_{1}\right) \in L^{2}(\mathbb{R}),\left\|\tilde{\psi}_{ \pm, \gamma_{1}}\left(\lambda_{1}\right)\right\|_{2}^{2}=\gamma_{1}^{-1}\left\{1-\lim _{x \rightarrow \mp \infty}\left[1 \mp \gamma_{1} \int_{ \pm \infty}^{x} d x^{\prime} \psi_{ \pm}\left(\lambda_{1}, x^{\prime}\right)^{2}\right]^{-1}\right\}
$$

$$
\begin{aligned}
& \mp \int_{ \pm \infty}^{x} d x^{\prime} \overline{\tilde{f}_{ \pm, \gamma_{1}}\left(\lambda_{1}, x^{\prime}\right)} \tilde{g}_{ \pm, \gamma_{1}}\left(\lambda_{1}, x^{\prime}\right)=\mp \int_{ \pm \infty}^{x} d x^{\prime} \overline{f\left(x^{\prime}\right)} g\left(x^{\prime}\right) \\
& \pm \gamma_{1}\left[1 \mp \gamma_{1} \int_{ \pm \infty}^{x} d x^{\prime} \dot{\psi}_{ \pm}\left(\lambda_{1}, x^{\prime}\right)^{2}\right]^{-1} \int_{ \pm \infty}^{x} d x^{\prime} \psi_{ \pm}\left(\lambda_{1}, x^{\prime}\right) \overline{f\left(x^{\prime}\right)} \int_{ \pm \infty}^{x} d x^{\prime \prime} \dot{\psi}_{ \pm}\left(\lambda_{1}, x^{\prime \prime}\right) g\left(x^{\prime \prime}\right)
\end{aligned}
$$

$$
\mp \int_{ \pm \infty}^{x} d x^{\prime} \overline{f\left(x^{\prime}\right)} g\left(x^{\prime}\right)=\mp \int_{ \pm \infty}^{x} d x^{\prime} \overline{\tilde{f}_{ \pm, \gamma_{1}}\left(\lambda_{1}, x^{\prime}\right)} \tilde{g}_{ \pm, \gamma_{1}}\left(\lambda_{\mathrm{l}}, x^{\prime}\right)
$$

(B.11) $\mp \gamma_{1}\left[1 \pm \gamma_{1} \int_{ \pm \infty}^{x} d x^{\prime} \tilde{\psi}_{ \pm, \gamma_{1}}\left(\lambda_{1}, x^{\prime}\right)^{2}\right]^{-1} \int_{ \pm \infty}^{x} d x^{\prime} \tilde{\psi}_{ \pm, \gamma_{1}}\left(\lambda_{1}, x^{\prime}\right) \overline{\tilde{\psi}_{ \pm, \gamma_{1}}\left(\lambda_{1}, x^{\prime}\right)}$

$$
\times \int_{ \pm \infty}^{x} d x^{\prime \prime} \tilde{\psi}_{ \pm, \gamma_{1}}\left(\lambda_{1}, x^{\prime \prime}\right) \tilde{g}_{ \pm, \gamma_{1}}\left(\lambda_{1}, x^{\prime \prime}\right)
$$

Next, we denote the restriction of $\hat{U}_{ \pm, \gamma_{1}}\left(\lambda_{1}\right)$ to $L^{2}(\mathbb{R})$ by

$$
U_{ \pm, \gamma_{1}}\left(\lambda_{1}\right)=\left.\widehat{U}_{ \pm, \gamma_{1}}\left(\lambda_{1}\right)\right|_{L^{2}(\mathbb{R})},
$$

define the orthogonal projections

$$
\begin{gathered}
P_{ \pm}\left(\lambda_{1}\right)= \begin{cases}0, & \dot{\psi}_{ \pm}\left(\lambda_{1}\right) \notin L^{2}(\mathbb{R}), \\
\left\|\psi_{ \pm}\left(\lambda_{1}\right)\right\|_{2}^{-2}\left(\psi_{ \pm}\left(\lambda_{1}\right), \cdot\right) \psi_{ \pm}\left(\lambda_{1}\right), & \dot{\psi}_{ \pm}\left(\lambda_{1}\right) \in L^{2}(\mathbb{R}),\end{cases} \\
\tilde{P}_{ \pm, \gamma_{1}}\left(\lambda_{1}\right)=\left\|\tilde{\psi}_{ \pm, \gamma_{1}}\left(\lambda_{1}\right)\right\|_{2}^{-2}\left(\tilde{\psi}_{ \pm, \gamma_{1}}\left(\lambda_{1}\right), \cdot\right) \tilde{\psi}_{ \pm, \gamma_{1}}\left(\lambda_{1}\right),
\end{gathered}
$$


and introduce the double commutation differential expression

$$
\begin{gathered}
\tilde{\tau}_{ \pm, \gamma_{1}}\left(\lambda_{1}\right)=-\frac{d^{2}}{d x^{2}}+\tilde{V}_{ \pm, \gamma_{1}}\left(\lambda_{1}, x\right), \quad x \in \mathbb{R} \\
\tilde{V}_{ \pm, \gamma_{1}}\left(\lambda_{1}, x\right)=V(x)-2\left\{\ln \left[1 \mp \gamma_{1} \int_{ \pm \infty}^{x} d x^{\prime} \psi_{ \pm}\left(\lambda_{1}, x^{\prime}\right)^{2}\right]\right\}^{\prime \prime}
\end{gathered}
$$

Relations (B.5)-(B.11) then yield

\section{Lemma B.1 [27]}

(i) $U_{ \pm, \gamma_{1}}\left(\lambda_{1}\right):\left(1-P_{ \pm}\left(\lambda_{1}\right)\right) L^{2}(\mathbb{R}) \rightarrow\left(1-\tilde{P}_{ \pm, \gamma_{1}}\left(\lambda_{1}\right)\right) L^{2}(\mathbb{R})$ is unitary.

(ii) $\tilde{\tau}_{ \pm, \gamma_{1}}\left(\lambda_{1}\right)\left(\widehat{U}_{ \pm, \gamma_{1}}\left(\lambda_{1}\right) f\right)=\widehat{U}_{ \pm, \gamma_{1}}\left(\lambda_{1}\right)(\tau f)$.

Lemma B.1(ii) shows that $U_{ \pm, \gamma_{1}}\left(\lambda_{1}\right)$ are transformation operators for the pairs $\left(\tilde{H}_{ \pm, \gamma_{1}}\left(\lambda_{1}\right), H_{ \pm}\right)$in the terminology of [37], Chapter 1, [39], Chapter 1, that is, they map solutions of $\tau \psi(z)=z \psi(z), z \in \mathbb{C} \backslash\left\{\lambda_{1}\right\}$ into those of $\tilde{\tau}_{ \pm, \gamma_{1}}\left(\lambda_{1}\right) \tilde{\psi}(z)=z \tilde{\psi}(z)$.

The self-adjoint operator $\tilde{H}_{ \pm, \gamma_{1}}\left(\lambda_{1}\right)$ corresponding to $\tilde{\tau}_{ \pm, \gamma_{1}}\left(\lambda_{1}\right)$ is then defined by

$$
\begin{aligned}
& \tilde{H}_{ \pm, \gamma_{1}}\left(\lambda_{1}\right) f=\tilde{\tau}_{ \pm, \gamma_{1}}\left(\lambda_{1}\right) f, \\
& f \in \mathcal{D}\left(\tilde{H}_{ \pm, \gamma_{1}}\left(\lambda_{1}\right)\right)=\left\{g \in L^{2}(\mathbb{R}) \mid g, g^{\prime} \in A C_{\mathrm{loc}}(\mathbb{R}) ; \tilde{\tau}_{ \pm, \gamma_{1}}\left(\lambda_{1}\right) g \in L^{2}(\mathbb{R}) ;\right. \\
& \lim _{x \rightarrow \omega \infty} W\left(\tilde{\psi}_{ \pm, \gamma_{1}}\left(\lambda_{1}\right), g\right)(x)=0 \\
& \text { if } \left.\tilde{\tau}_{ \pm, \gamma_{l}}\left(\lambda_{1}\right) \text { is l.c. at } \omega \infty, \omega \in\{-,+\}\right\} \text {. }
\end{aligned}
$$

As usual, the last boundary condition at $\omega \infty$ in (B.15) is to be deleted if $\tilde{\tau}_{ \pm, \gamma_{1}}\left(\lambda_{1}\right)$ is l.p. at $\omega \infty$.

The principal result concerning the spectra of $\tilde{H}_{ \pm, \gamma_{1}}\left(\lambda_{1}\right)$ and $H_{ \pm}$then reads as follows.

Theorem B.2 [27] Let $H_{ \pm}, \tilde{H}_{ \pm, \gamma_{1}}\left(\lambda_{1}\right), \gamma_{1}>0, \psi_{ \pm}\left(\lambda_{1}, x\right), \tilde{\psi}_{ \pm, \gamma_{1}}\left(\lambda_{1}, x\right), \lambda_{1} \in \mathbb{R}$, and $U_{ \pm, \gamma_{1}}\left(\lambda_{1}\right), P_{ \pm}\left(\lambda_{1}\right), \tilde{P}_{ \pm, \gamma_{1}}\left(\lambda_{1}\right)$ be given as in (B.3), (B.15), (B.4), (B.6), and (B.12), (B.13). Then

(i) $\lambda_{1} \in \sigma_{p}\left(\tilde{H}_{ \pm, \gamma_{1}}\left(\lambda_{1}\right)\right)$ and

$$
\operatorname{Ker}\left(\tilde{H}_{ \pm, \gamma_{1}}\left(\lambda_{1}\right)-\lambda_{1}\right)=\operatorname{span}\left\{\tilde{\psi}_{ \pm, \gamma_{1}}\left(\lambda_{1}\right)\right\}
$$

(ii) If $\psi_{ \pm}\left(\lambda_{1}\right) \notin L^{2}(\mathbb{R})$ (and hence $\tau$ is l.p. at $\pm \infty$ ), one obtains

$$
\tilde{H}_{ \pm, \gamma_{1}}\left(\lambda_{1}\right)\left(1-\tilde{P}_{ \pm, \gamma_{1}}\left(\lambda_{1}\right)\right)=U_{ \pm, \gamma_{1}}\left(\lambda_{1}\right) H U_{ \pm, \gamma_{1}}\left(1-\tilde{P}_{ \pm, \gamma_{1}}\left(\lambda_{1}\right)\right)
$$


that is, $\tilde{H}_{ \pm, \gamma_{1}}\left(\lambda_{1}\right)$ and $H$, restricted to the orthogonal complement of the (onedimensional) eigenspace associated with the eigenvalue $\lambda_{1}$ of $\tilde{H}_{ \pm, \gamma_{1}}\left(\lambda_{1}\right)$, are unitarily equivalent. In particular.

$$
\sigma_{(p)}\left(\tilde{H}_{ \pm, \gamma_{1}}\left(\lambda_{1}\right)\right)=\sigma_{(p)}(H) \cup\left\{\lambda_{1}\right\}
$$

(iii) If $\psi_{ \pm}\left(\lambda_{1}\right) \in L^{2}(\mathbb{R})$, then there exists a unitary operator $\tilde{U}_{ \pm, \gamma_{1}}\left(\lambda_{1}\right)=$ $U_{ \pm, \gamma_{1}}\left(\lambda_{1}\right) \oplus \sqrt{1+\gamma_{1}\left\|\psi_{ \pm}\left(\lambda_{1}\right)\right\|_{2}^{2}} 1$ on $\left(1-P_{ \pm}\left(\lambda_{1}\right)\right) L^{2}(\mathbb{R}) \oplus P_{ \pm}\left(\lambda_{1}\right) L^{2}(\mathbb{R})$ such that

$$
\tilde{H}_{ \pm, \gamma_{1}}\left(\lambda_{1}\right)=\tilde{U}_{ \pm, \gamma_{1}}\left(\lambda_{1}\right) H_{ \pm} \tilde{U}_{ \pm, \gamma_{1}}\left(\lambda_{1}\right)^{-1}
$$

Moreover,

$$
\sigma_{\mathrm{ess}, \mathrm{ac}, \mathrm{sc}}\left(\tilde{H}_{ \pm, \gamma_{1}}\left(\lambda_{1}\right)\right)=\sigma_{\mathrm{ess}, \mathrm{ac}, \mathrm{sc}}\left(H_{ \pm}\right)
$$

in cases (ii) and (iii).

Remark B.3 (i) Thus far we considered the case $0<\gamma_{ \pm}<\infty$. The limit $\gamma_{ \pm} \rightarrow \infty$ in (B.14) (implying $\tilde{\psi}_{ \pm, \gamma_{1}}\left(\lambda_{1}, x\right) \underset{\gamma_{1} \rightarrow \infty}{\longrightarrow} 0$ and hence $\tilde{P}_{ \pm, \gamma_{1}}\left(\lambda_{1}\right)=0$ ) formally seems to yield an isospectral deformation of $H_{ \pm}$when compared with Theorem B.2. One computes

$$
\begin{aligned}
\tilde{\tau}_{ \pm, \infty}\left(\lambda_{1}\right) & =-\frac{d^{2}}{d x^{2}}+\tilde{V}_{ \pm, \infty}\left(\lambda_{1}, x\right), \quad x \in \mathbb{R} \\
\tilde{V}_{ \pm, \infty}\left(\lambda_{1}, x\right) & =V(x)-2\left\{\ln \left[\mp \int_{ \pm \infty}^{x} d x^{\prime} \psi_{ \pm}\left(\lambda_{1}, x^{\prime}\right)^{2}\right]\right\}^{\prime \prime}
\end{aligned}
$$

A quick look at (2.19) and (2.20) then shows that (B.21) is precisely the (sign flip) Dirichlet deformation (cf. Remark 4.6(iii)) where

$$
\left(\lambda_{1}, \pm\right) \rightarrow\left(\lambda_{1}, \mp\right), \quad \tilde{\tau}_{ \pm, \infty}\left(\lambda_{1}\right)=\tilde{\tau}_{\left(\lambda_{1}, \mp\right)}, \quad \mu=\tilde{\mu}=\lambda_{1}, \quad \tilde{\sigma}=-\sigma=\mp
$$

As a consequence of (B.22), Cases II and III in (2.34) and (2.35) coincide and the boundary conditions in $\tilde{H}_{\left(\lambda_{1}, \mp\right)}$ (if any) are identical to those in (B.15), upon replacing $\tilde{\psi}_{ \pm, \gamma_{1}}\left(\lambda_{1}, x\right)$ by $\gamma_{1} \tilde{\psi}_{ \pm, \gamma_{1}}\left(\lambda_{1}, x\right)$ and formally letting $\gamma_{1} \rightarrow \infty$. Thus

$$
\tilde{H}_{\omega, \infty}\left(\lambda_{1}\right)=\tilde{H}_{\left(\lambda_{1},-\omega\right)}, \quad\left(\lambda_{1}, \omega\right)=(\mu, \omega)=(\tilde{\mu},-\omega), \omega \in\{-,+\}
$$

is the right definition for the self-adjoint operator associated with $\bar{\tau}_{\omega, \infty}\left(\lambda_{1}\right)$ in (B.21). Hence the case $\gamma_{1}=\infty$ is fully covered by Sections 3-5, and (B.21) indeed gives rise to an isospectral deformation of $H_{ \pm}$. The isospectral nature of (B.21) 
has been systematically exploited in the context of Bäcklund transformations for the $(\mathrm{m}) \mathrm{KdV}$ equation in [26].

(ii) If $H_{ \pm}$has an eigenfunction $\psi_{ \pm}\left(\lambda_{1}\right) \in \mathcal{D}\left(H_{ \pm}\right)$associated with the eigenvalue $\lambda_{1}$, one can reverse DCM and remove $\lambda_{1}$ upon choosing $\gamma_{1}=-\left\|\psi_{ \pm}\left(\lambda_{1}\right)\right\|_{2}^{-2}$. In this case, $\tilde{\psi}_{ \pm, \gamma_{1}}\left(\lambda_{1}\right) \notin L^{2}(\mathbb{R})$ and hence $\tilde{\tau}_{ \pm, \gamma_{1}}\left(\lambda_{1}\right)$ is l.p. at $\pm \infty$ (cf. [19], [27]).

(iii) Similarly to DDM (and in contrast to SCM in Appendix A where we exploited that $\tau$ was non-oscillatory and hence l.p. at $\pm \infty$ ), DCM does not necessarily produce a l.p. differential expression $\tilde{\tau}_{ \pm, \gamma_{1}}\left(\lambda_{1}\right)$ at $\mp \infty$ even if $\tau$ was 1.p. at $\mp \infty$. In fact, one can use (ii) above to construct an example where $\tau$ is 1.p. at $\mp \infty$ but $\tilde{\tau}_{ \pm, \gamma_{1}}\left(\lambda_{1}\right)$ is l.c. at $\mp \infty$ ([19], [27]). However, $\tilde{\tau}_{ \pm, \gamma_{1}}\left(\lambda_{1}\right)$ is l.p. at $\pm \infty$ if and only if $\tau$ is and $\tilde{\tau}_{ \pm . \gamma_{1}}\left(\lambda_{1}\right)$ is l.c. at $\mp \infty$ if $\tau$ is ([19], [27]). Of course, Lemma 5.1 immediately covers the present situation upon entering the obvious changes in notation.

Next, we turn to a computation of Weyl-Titchmarsh functions associated with $\tilde{H}_{ \pm, \gamma_{1}}\left(\lambda_{1}\right)$ in terms of those of $H_{ \pm}$. Since some of the following results (such as (B.26)) are new, we provide a few more details. First, some necessary notation. Let $x_{0} \in \mathbb{R}$ be a fixed reference point and assume temporarily the notation used in Lemma 3.1. Given $\widehat{H}, \widehat{V}$, and $\widehat{m}_{ \pm}(z)$, the corresponding $m$-functions associated with the half-line $\left(x_{0}, \pm \infty\right)$, we define the usual fractional linear transformation of $\widehat{m}_{ \pm}(z)$

$$
\begin{aligned}
& \hat{m}_{ \pm}^{\alpha}(z)=\frac{-1+\cot (\alpha) \widehat{m}_{ \pm}(z)}{\cot (\alpha)+\widehat{m}_{ \pm}(z)}, \quad \alpha \in(0, \pi) \\
& \hat{m}_{ \pm}^{0}(z)=\widehat{m}_{ \pm}(z), \quad z \in \mathbb{C} \backslash \mathbb{R}
\end{aligned}
$$

and

$$
\begin{aligned}
\hat{M}^{\alpha}(z) & =\left(\begin{array}{ll}
\cos (\alpha) & -\sin (\alpha) \\
\sin (\alpha) & \cos (\alpha)
\end{array}\right) \widehat{M}(z)\left(\begin{array}{ll}
\cos (\alpha) & -\sin (\alpha) \\
\sin (\alpha) & \cos (\alpha)
\end{array}\right)^{-1} \\
& =\left[\hat{m}_{-}^{\alpha}(z)-\hat{m}_{+}^{\alpha}(z)\right]^{-1}\left(\begin{array}{ll}
\hat{m}_{-}^{\alpha}(z) \hat{m}_{+}^{\alpha}(z) & \frac{1}{2}\left[\widehat{m}_{-}^{\alpha}(z)+\hat{m}_{+}^{\alpha}(z)\right] \\
\frac{1}{2}\left[\widehat{m}_{-}^{\alpha}(z)+\widehat{m}_{+}^{\alpha}(z)\right] & 1
\end{array}\right), \\
\widehat{M}^{0}(z) & =\widehat{M}(z), \quad z \in \mathbb{R}
\end{aligned}
$$

with $\widehat{M}(z)$ defined in terms of $\widehat{m}_{ \pm}(z)$ as in (4.1). (B.24) and (B.25) are associated with the boundary condition $\sin (\alpha) \psi^{\prime}\left(x_{0} \pm 0\right)+\cos (\alpha) \psi\left(x_{0} \pm 0\right)=0, \alpha \in(0, \pi)$ as opposed to the Dirichlet boundary condition $\alpha=0, \psi\left(x_{0} \pm 0\right)=0$ in connection with $\hat{m}_{ \pm}(z)$ and $\widehat{M}(z)$.

Lemma B.4 Denote by $\tilde{m}_{\omega, \gamma_{1}, \pm}^{\beta}\left(z, \lambda_{1}\right)$ and $m_{ \pm}^{\alpha}(z)$ the corresponding $m$-functions for $\tilde{H}_{\omega, \gamma_{1}}\left(\lambda_{1}\right), \omega \in\{-,+\}$ and $H\left(=H_{\omega}\right)$ associated with the half-line $\left(x_{0}, \pm \infty\right)$. 
(i) Suppose $m_{\omega}^{0}\left(\lambda_{1}\right) \neq \infty$ (i.e., $\left.\psi_{\omega}\left(\lambda_{1}, x_{0}\right) \neq 0\right), \omega \in\{-,+\}$. Then

(B.26)

$$
\begin{aligned}
& \tilde{m}_{\omega, \gamma_{1}, \pm}^{\beta_{\omega}\left(\lambda_{1}\right)}\left(z, \lambda_{1}\right)=\cot \left(\beta_{\omega}\left(\lambda_{1}\right)\right) \\
& +\frac{\sin ^{2}\left(\alpha_{\omega}\left(\lambda_{1}\right)\right)}{\sin ^{2}\left(\beta_{\omega}\left(\lambda_{1}\right)\right)}\left[m_{ \pm}^{\alpha_{\omega}\left(\lambda_{1}\right)}(z)-\cot \left(\alpha_{\omega}\left(\lambda_{1}\right)\right)+\frac{\omega \bar{\gamma}_{1}\left(\psi_{\omega}\left(\lambda_{1}, x_{0}\right)^{2}+\psi_{\omega}^{\prime}\left(\lambda_{1}, x_{0}\right)^{2}\right)}{z-\lambda_{1}}\right] \\
& \tilde{\gamma}_{1}=\gamma_{1}\left[1-\omega \gamma_{1} \int_{\omega \infty}^{x_{0}} d x \psi_{\omega}\left(\lambda_{1}, x\right)^{2}\right]^{-1}, \\
& \cot \left(\alpha_{\omega}\left(\lambda_{1}\right)\right)=-m_{\omega}^{0}\left(\lambda_{1}\right), \cot \left(\beta_{\omega}\left(\lambda_{1}\right)\right)=\cot \left(\alpha_{\omega}\left(\lambda_{1}\right)\right)-\omega \tilde{\gamma}_{1} \psi_{\omega}\left(\lambda_{1}, x_{0}\right)^{2} .
\end{aligned}
$$

(ii) Suppose $m_{\omega}^{0}\left(\lambda_{1}\right)=\infty$ (i.e., $\left.\psi_{\omega}\left(\lambda_{l}, x_{0}\right)=0\right), \omega \in\{-,+\}$. Then

$$
\tilde{m}_{\omega, \gamma_{1}, \pm}^{0}\left(z, \lambda_{1}\right)=m_{ \pm}^{0}(z)+\frac{\omega \tilde{\gamma}_{1} \psi_{\omega}^{\prime}\left(\lambda_{1}, x_{0}\right)^{2}}{z-\lambda_{1}}
$$

with $\bar{\gamma}_{1}$ as in (B.26).

Proof We recall that

$$
\tilde{\psi}_{\omega, \gamma_{1}, \pm}\left(z, \lambda_{1}, x\right)=\dot{\psi}_{ \pm}(z, x)-\omega \gamma_{1} \frac{\tilde{\psi}_{\omega, \gamma_{1}}\left(\lambda_{1}, x\right)}{z-\lambda_{1}} W\left(\dot{\psi}_{\omega}\left(\lambda_{1}\right), \dot{\psi}_{ \pm}(z)\right)(x)
$$

satisfies

$$
\begin{gathered}
\tilde{\tau}_{\omega, \gamma_{1}}\left(\lambda_{1}\right) \tilde{\psi}_{\omega, \gamma_{1} . \pm}\left(z, \lambda_{1}\right)=z \tilde{\psi}_{\omega, \gamma_{1},}=\left(z, \lambda_{1}\right), \\
\tilde{\psi}_{\omega, \gamma_{1}, \pm}\left(z, \lambda_{1}, \cdot\right) \in L^{2}((R, \pm \infty)), \quad z \in \mathbb{C} \backslash \sigma\left(\tilde{H}_{\omega, \gamma_{1}}\left(\lambda_{1}\right)\right), \quad R \in \mathbb{R}
\end{gathered}
$$

and also note that

$$
\begin{aligned}
\tilde{\psi}_{\omega, \gamma_{1}, \omega}\left(\lambda_{1}, \lambda_{1}, x\right)= & \tilde{\psi}_{\omega, \gamma_{1}}\left(\lambda_{1}, x\right)=\left[1-\omega \gamma_{1} \int_{\omega \infty}^{x} d x^{\prime} \psi_{\omega}\left(\lambda_{1}, x^{\prime}\right)^{2}\right]^{-1} \psi_{\omega}\left(\lambda_{1}, x\right), \\
& \left.\frac{d}{d x} W\left(\dot{\psi}_{\omega_{1}}\left(z_{1}\right), \dot{\psi}_{\omega_{2}}\left(z_{2}\right)\right)(x)\right|_{x=x_{0}}=z_{1}-z_{2},
\end{aligned}
$$

$$
\begin{gathered}
m_{\omega}^{0}(z)=\psi_{\omega}^{\prime}\left(z, x_{0}\right) / \psi_{\omega}\left(z, x_{0}\right) \\
W\left(\psi_{\omega_{1}}\left(z_{1}\right), \psi_{\omega_{2}}\left(z_{2}\right)\right)\left(x_{0}\right)=m_{\omega_{2}}^{0}\left(z_{2}\right)-m_{\omega_{1}}^{0}\left(z_{1}\right) .
\end{gathered}
$$

(B.29) and (B.30) then yield

(B.31)

$$
\begin{aligned}
\tilde{m}_{\omega, \gamma_{1}, \pm}^{0}\left(z, \lambda_{1}\right)= & \tilde{\psi}_{\omega, \gamma_{1}, \pm}^{\prime}\left(z, \lambda_{1}, x_{0}\right) / \tilde{\psi}_{\omega, \gamma_{1}, \pm}\left(z, \lambda_{1}, x_{0}\right) \\
= & \left\{1-\omega \tilde{\gamma}_{1} \psi_{\omega}\left(\lambda_{1}, x_{0}\right)^{2}\left(m_{ \pm}^{0}(z)-m_{\omega}^{0}\left(\lambda_{1}\right)\right)\left(z-\lambda_{1}\right)^{-1}\right\}^{-1} \\
& \times\left\{m_{ \pm}^{0}(z)+\omega \tilde{\gamma}_{1} \dot{\psi}_{\omega}\left(\lambda_{1}, x_{0}\right)^{2}-\omega \tilde{\gamma}_{1} \psi_{\omega}\left(\lambda_{1}, x_{0}\right)^{2}\right. \\
& \left.\times\left[\left(m_{\omega}^{0}\left(\lambda_{1}\right)+\omega \tilde{\gamma}_{1} \psi_{\omega}\left(\lambda_{1}, x_{0}\right)^{2}\right)\left(m_{ \pm}^{0}(z)-m_{\omega}^{0}\left(\lambda_{1}\right)\right)\right]\left(z-\lambda_{1}\right)^{-1}\right\}
\end{aligned}
$$




$$
\text { if } m_{\omega}^{0}\left(\lambda_{1}\right) \neq \infty
$$

and (B.27) by a limiting procedure if $m_{\omega}^{0}\left(\lambda_{1}\right)=\infty$. Applying (B.24) to $m_{ \pm}^{0}(z)$ and $\tilde{m}_{\omega, \gamma_{1}, \pm}^{0}\left(z, \lambda_{1}\right)$ with the choices

$$
\cot \left(\alpha_{\omega}\left(\lambda_{1}\right)\right)=-m_{\omega}^{0}\left(\lambda_{1}\right) \text { and } \cot \left(\beta_{\omega}\left(\lambda_{1}\right)\right)=\cot \left(\alpha_{\omega}\left(\lambda_{1}\right)\right)-\omega \tilde{\gamma}_{1} \psi_{\omega}\left(\lambda_{1}, x_{0}\right)^{2}
$$

then shows, by a straightforward (but somewhat painful) computation, that (B.31) is equivalent to (B.26).

The singularity structure of (B.26) and (B.27) near $z=\lambda_{1}$ then leads to a corresponding pole behavior of $\tilde{M}_{\omega, \gamma_{1}}^{\beta_{\omega}\left(\lambda_{1}\right)}\left(z, \lambda_{1}\right), \tilde{M}_{\omega, \gamma_{1}}^{0}\left(z, \lambda_{1}\right)$ (the $\mathbb{C}^{2}$-valued $M$-matrices of $\tilde{H}_{\omega, \gamma_{1}}\left(\lambda_{1}\right)$ ) when compared to $M^{\alpha_{\omega}\left(\lambda_{1}\right)}(z), M^{0}(z)$ (the $M$-matrices of $H$ ). The actual expressions for the $M$-matrices, the half-line spectral functions, and the $\mathbb{C}^{2}$-valued spectral matrix of $\tilde{H}_{\omega, \gamma_{1}}\left(\lambda_{1}\right)$ for $\alpha_{\omega} \neq 0, \beta_{\omega} \neq 0$ in terms of those of $H$ are similar to the special case $\alpha_{\omega}=\beta_{\omega}=0$ and $m_{\omega_{0}}\left(\lambda_{1}\right)=\infty$ described in detail in [19]. While they provide an alternative proof of Theorem B.2, we resist the temptation of providing detailed formulas at this point. (B.26) appears to be a new result.

Iterations of DCM can now be performed as follows. Assume $V \in L_{\text {loc }}^{1}(\mathbb{R})$ to be real-valued and pick $\omega \in\{-,+\}, \gamma_{j}>0, \lambda_{j} \in \mathbb{R}, 1 \leq j \leq N, N \in \mathbb{N}$. Then the DCM result after $N$ iteration steps, denoted by $\tilde{\tau}_{\omega, \gamma_{1}, \ldots, \gamma_{N}}\left(\lambda_{1}, \ldots, \lambda_{N}\right)$, reads as follows:

$$
\begin{gathered}
\tilde{\tau}_{\omega, \gamma_{1}, \ldots, \gamma_{N}}\left(\lambda_{1}, \ldots, \lambda_{N}\right)=-\frac{d^{2}}{d x^{2}}+\tilde{V}_{\omega, \gamma_{1}, \ldots, \gamma_{N}}\left(\lambda_{1}, \ldots, \lambda_{N}, x\right), \quad x \in \mathbb{R}, \\
\tilde{V}_{\omega, \gamma_{1}, \ldots, \gamma_{N}}\left(\lambda_{1}, \ldots, \lambda_{N}, x\right)=V(x)-2\left\{\ln \left[\operatorname{det}\left(1+C_{\omega, N}(x)\right)\right]\right\}^{\prime \prime}, \\
C_{\omega, N}(x)=\left(-\omega \gamma_{k}^{1 / 2} \gamma_{\ell}^{1 / 2} \int_{\omega \infty}^{x} d x^{\prime} \dot{\psi}_{\omega}\left(\lambda_{k}, x^{\prime}\right) \dot{\psi}_{\omega}\left(\lambda_{\ell}, x^{\prime}\right)\right)_{1 \leq k, \ell \leq N} .
\end{gathered}
$$

The analog of Theorem B.2 then applies to $\tilde{H}_{\omega, \gamma_{1}, \ldots, \gamma_{N}}\left(\lambda_{1}, \ldots, \lambda_{N}\right)$, the self-adjoint operator associated with $\tilde{\tau}_{\omega, \gamma_{1}, \ldots, \gamma_{N}}\left(\lambda_{1}, \ldots, \lambda_{N}\right)$ (defined similarly to (B.15)) as discussed in detail in [27] (see also [22], [26]).

As in Lemma 5.4 one infers from (B.31) that $\tilde{H}_{\omega, \gamma_{1}}\left(\lambda_{1}\right)$ is reflectionless if and only if $H$ is (as observed in [21]).

The Sturm-Liouville generalization of (B.1)-(B.14) then leads to the following results. One assumes

$$
\begin{array}{r}
p^{-1}, q, k \in L_{\mathrm{loc}}^{1}((a, b)), \quad k p \in A C_{\mathrm{loc}}((a, b)), \quad q \text { real-valued, } \\
p>0, k>0, \quad-\infty \leq a<b \leq \infty
\end{array}
$$


and makes the substitutions (see [27])

$$
\begin{gathered}
\tau \rightarrow \widehat{\tau}=\frac{1}{k(x)}\left(-\frac{d}{d x} p(x) \frac{d}{d x}+q(x)\right), \\
\tilde{\tau}_{\omega, \gamma_{1}}\left(\lambda_{1}\right) \rightarrow \widetilde{\widetilde{\tau}}_{\omega, \gamma_{1}}\left(\lambda_{1}\right)=\frac{1}{k(x)}\left(-\frac{d}{d x} p(x) \frac{d}{d x}+\tilde{q}_{\omega, \gamma_{1}}\left(\lambda_{1}, x\right)\right), \\
\tilde{q}_{\omega, \gamma_{1}}\left(\lambda_{1}, x\right)=q(x)+\left(\frac{1}{k(x)}(k(x) p(x))^{\prime}-2 \frac{d}{d x} p(x)\right) \\
\times \frac{d}{d x} \ln \left[1-\omega \gamma_{1} \int_{\omega \infty}^{x} k\left(x^{\prime}\right) d x^{\prime} \dot{\psi}_{\omega}\left(\lambda_{1}, x^{\prime}\right)^{2}\right], \omega \in\{-,+\} .
\end{gathered}
$$

It remains to sketch scattering theory for real-valued potentials $V \in$ $L^{\prime}(\mathbb{R} ;(1+|x|) d x)$ similar to DDM and SCM in Section 5 and Appendix A. (We again use the conventions established in (5.11)-(5.17).) Suppose $\lambda_{1}<0, \gamma_{1}>0$. Then we claim that DCM leaves $L^{1}(\mathbb{R} ;(1+|x|) d x)$ potentials invariant as in DDM and SCM. More precisely, we assert that $\tilde{V}_{\omega, \gamma_{1}}\left(\lambda_{1}\right) \in L^{1}(\mathbb{R} ;(1+|x|) d x)$ if and only if $V \in L^{\prime}(\mathbb{R} ;(1+|x|) d x)$. Indeed, since

$$
\begin{aligned}
& \tilde{V}_{\omega, \gamma_{1}}\left(\lambda_{1}, x\right)-V(x)=\left[1-\omega \gamma_{1} \int_{\omega \infty}^{x} d x^{\prime} \psi_{\omega}\left(\lambda_{1}, x^{\prime}\right)^{2}\right]^{-2} 2 \gamma_{1}^{2} \psi_{\omega}\left(\lambda_{1}, x\right)^{4} \\
& +\left[1-\omega \gamma_{1} \int_{\omega \infty}^{x} d x^{\prime} \dot{\psi}_{\omega}\left(\lambda_{1}, x^{\prime}\right)^{2}\right]^{-1} 4 \omega \gamma_{1} \dot{\psi}_{\omega}\left(\lambda_{1}, x\right) \dot{\psi}_{\omega}^{\prime}\left(\lambda_{1}, x\right), \quad \omega \in\{-,+\},
\end{aligned}
$$

the right-hand side of (B.34) is exponentially decreasing near $\omega \infty$ and hence in $L^{1}((R, \omega \infty) ;(1+|x|) d x)$ for all $R \in \mathbb{R}$. In order to treat (B.34) near $-\omega \infty$, one expands

$$
\begin{aligned}
{\left[\gamma_{1}^{-1}-\omega \int_{\omega \infty}^{x} d x^{\prime} \psi_{\omega}\left(\lambda_{1}, x^{\prime}\right)^{2}\right]^{-1} \underset{x \rightarrow-\omega \infty}{=} } & \left(-\omega \int_{\omega \infty}^{x} d x^{\prime} \psi_{\omega}\left(\lambda_{1}, x^{\prime}\right)^{2}\right)^{-1} \\
\times & {\left[1+o\left(\left(-\omega \int_{\omega \infty}^{x} d x^{\prime} \dot{\psi}_{\omega}\left(\lambda_{1}, x^{\prime}\right)^{2}\right)^{-1}\right)\right] }
\end{aligned}
$$

in (B.34) and notices that $O\left((\ldots)^{-1}\right)$ is exponentially decreasing as $x \rightarrow-\omega \infty$. The proof of our assertion is then finished by observing that the leading order term in (B.34) is precisely the isospectral double commutation deformation corresponding to $\gamma_{1}=\infty$ (cf. Remark B.4(i)), which in turn corresponds to the (sign flip) Dirichlet deformation $\left(\lambda_{1},+\omega\right) \rightarrow\left(\lambda_{1},-\omega\right)$. The latter has been dealt with in Lemma 5.5. 
The fact that DCM leaves $L^{1}(\mathbb{R} ;(1+|x|) d x)$ potentials invariant was proved by Levitan [37], Section 6.6 using a different strategy (which yields exponential decay of $\left[\tilde{V}_{\omega, \gamma_{1}}\left(\lambda_{1}, x\right)-V(x)\right]$ also as $\left.x \rightarrow-\omega \infty\right)$.

Finally, we compute the scattering matrix in this context. Following the arguments in (5.11)-(5.20), one readily verifies the following expressions for the scattering matrix $\tilde{S}_{\omega, \gamma_{1}}\left(\lambda, \lambda_{1}\right)$ of the pair $\left(\tilde{H}_{\omega, \gamma_{1}}\left(\lambda_{1}\right), H_{0}\right)$ in terms of $S(\lambda)$ corresponding to $\left(H, H_{0}\right)$ :

$$
\begin{aligned}
& \tilde{T}_{\omega, \gamma_{1}}\left(\lambda, \lambda_{1}\right)=\frac{\lambda^{1 / 2}+i\left(-\lambda_{1}\right)^{1 / 2}}{\lambda^{1 / 2}-i\left(-\lambda_{1}\right)^{1 / 2}} T(\lambda), \quad \omega \in\{-,+\} \\
& \tilde{R}_{\omega, \gamma_{1}}^{\ell}\left(\lambda, \lambda_{1}\right)= \begin{cases}R^{\ell}(\lambda), & \omega=-, \\
\left(\frac{\lambda^{1 / 2}+i\left(-\lambda_{1}\right)^{1 / 2}}{\lambda^{1 / 2}-i\left(-\lambda_{1}\right)^{1 / 2}}\right)^{2} R^{\ell}(\lambda), & \omega=+,\end{cases} \\
& \tilde{R}_{\omega, \gamma_{1}}^{r}\left(\lambda, \lambda_{1}\right)= \begin{cases}\left(\frac{\lambda^{1 / 2}+i\left(-\lambda_{1}\right)^{1 / 2}}{\lambda^{1 / 2}-i\left(-\lambda_{1}\right)^{1 / 2}}\right)^{2} R^{r}(\lambda), & \omega=-, \\
R^{r}(\lambda), & \omega=+,\end{cases} \\
& \lambda>0, \lambda_{1} \in(-\infty, 0) \backslash \sigma_{d}(H), \gamma_{1}>0,
\end{aligned}
$$

$$
\begin{gathered}
\tilde{T}_{\omega, \gamma_{1}}\left(\lambda, \lambda_{1}\right)=T(\lambda), \quad \tilde{R}_{\omega, \gamma_{1}}^{\ell}\left(\lambda, \lambda_{1}\right)=R^{\ell}(\lambda), \\
\omega \in\{-,+\}, \lambda>0, \lambda_{1} \in \sigma_{d}(H), \gamma_{1}>0 .
\end{gathered}
$$

The case $\gamma_{1}=\infty$ is a special case of (5.19) with $\mu=\tilde{\mu}=\lambda_{1}, \sigma=-\tilde{\sigma}=\omega$.

The discrete analog of DCM for general second-order finite-difference (Jacobi) operators can be found in [28].

As in Appendix A, we conclude with a brief account of the history of DCM and references to further applications of it. The seminal work by Gel'fand and Levitan [16] in 1955 on a solution of the inverse spectral problem seems to mark the first appearance of DCM where it has been used in connection with Wigner-von Neumann examples on the half-line $(0, \infty)$. (For a more recent treatment of the half-line case $(0, \infty)$, see [7].) Shortly afterward, the construction of reflectionless potentials in the particular case of $\tau=-d^{2} / d x^{2}$ (i.e., $V \equiv 0$ ), using double commutation formulas as a result of applying the inverse scattering approach, was derived by Kay and Moses [34]. Their result regained prominence when Gardner, Greene, Kruskal, and Miura [15] used this formalism to solve the initial value problem for the $\mathrm{KdV}$ equation and derived the $\mathrm{KdV} N$-soliton solutions. The case of background (base) potentials $V \in L^{1}(\mathbb{R} ;(1+|x|) d x)$ is considered at length in [8] and [37], Section 6.6. The case of periodic finite-gap background potentials is treated in [13], [14], [26], and [35]. Close connections between the double commutation technique and the inverse spectral method based on Marchenko's approach can be inferred, for instance, from [13], [14], [15], [22], [34], and 
[35]. General backgrounds were first treated in [26] (see also [9], Chapter 4). In particular, the construction of $\mathrm{KdV}$ and $\mathrm{mKdV}$ soliton solutions relative to general $(\mathrm{m}) \mathrm{KdV}$ background solutions on the basis of (single and double) commutation techniques has been systematically studied in [26]. In spite of the widespread use of the double commutation method, its spectral characterization, as summarized in Theorem B.2, under slightly stronger assumptions on $\tau$, was first proven only recently in [19].

\section{REFERENCES}

[1] B. Baumgartner, Level comparison theorems, Ann. Phys. 168 (1986), 484-526.

[2] E. D. Belokolos, A. I. Bobenko, V. Z. Enol'skii, A. R. Its and V. B. Matveev, Algebro-Geometric Approach to Nonlinear Integrable Equations, Springer, Bcrlin, 1994.

[3] D. Bolle, F. Gesztesy, H. Grosse, W. Schweiger and B. Simon, Witten index, axial anomaly, and Krein's spectral shift function in supersymmetric quantum mechanics, J. Math. Phys. 28 (1987), $1512-1525$.

[4] M. Buys and A. Finkel, The inverse periodic problem for Hill's equation with a finite-gap potential, J. Differential Equations 55 (1984), 257-275.

[5] M. M. Crum, Associated Sturm-Liouville systems, Quart. J. Math. Oxford (2) 6 (1955), 121-127.

[6] G. Darboux, Sur une proposition relative aux équations linéaires, C. R. Acad. Sci. (Paris) 94 (1882), 1456-1459.

[7] P. A. Deift, Applications of a commutation formula, Duke Math. J. 45 (1978), 267-310.

[8] P. Deift and E. Trubowitz, Inverse scattering on the line, Comm. Pure Appl. Math. 32 (1979), $121-251$.

[9] M. S. P. Eastham and H. Kalf, Schrödinger-Type Operators with Continuous Spectra, Pitman, Boston, 1982.

[10] F. Ehlers and H. Knörrer, An algebro-geometric interpretation of the Bäcklund transformation of the Korteweg-de Vries equation, Comment. Math. Helv. 57 (1982), 1-10.

[11] N. M. Ercolani and H. Flaschka, The geometry of the Hill equation and of the Neumann system, Philos. Trans. Roy. Soc. London Ser. A 315 (1985), 405-422.

[12] A. Finkel, E. Isaacson and E. Trubowitz, An explicit solution of the inverse problem for Hill's equation, SIAM J. Math. Anal. 18 (1987), 46-53.

[13] N. E. Firsova, On solution of the Cauchy problem for the Korteweg-de Vries equation with initial data the sum of a periodic and a rapidly decreasing function, Math. USSR Sbornik 63 (1989), 257-265.

[14] H. Flaschka and D. W. McLaughlin, Some comments on Bäcklund transformations, canonical transformations, and the inverse scattering method, Lecture Notes in Math., Vol. 515 (R.M. Miura, ed.), Springer, Berlin, 1976, pp. 252-295.

[15] C. S. Gardner, J. M. Greenc, M. D. Kruskal and R. M. Miura, Korteweg-de Vries equation and generalizations, VI. Methods for exact solution, Comm. Pure Appl. Math. 27 (1974), 97-133.

[16] I. M. Gel'fand and B. M. Levitan, On the determination of a differential equation from its spectral function, Amer. Math. Transl. Ser. 21 (1955), 253-304.

[17] F. Gesztesy, On the modified Korteweg-de Vries equation, in Differential Equations with Applications in Biology, Physics, and Engineering (J.A. Goldstein, F. Kappel and W. Schappacher, eds.), Marcel Dekker, New York, 1991, pp. 139-183.

[18] F. Gesztesy, Quasi-periodic, finite-gap solutions of the modified Korteweg-de Vries equation, in Ideas and Methods in Mathematical Analysis, Stochastics, and Applications (S. Albeverio, J. E. Fenstad, H. Holden and T. Lindstrøm, eds.), Vol. 1, Cambridge Univ. Press, Cambridge, 1992, pp. 428-471.

[19] F. Gesztesy, A complete spectral characterization of the double commutation method J. Funct. Anal. 117 (1993), 401-446.

[20] F. Gesztesy, M. Krishna and G. Teschl, On isospectral sets of Jacobi operators, to appear in Comm. Math. Phys.

[21] F. Gesztesy, R. Nowell and W. Pötz, One-dimensional scattering theory for quantum systems with nontrivial spatial asymptotics, to appear in Adv. Differential Equations. 
[22] F. Gesztesy, W. Schweiger and B. Simon, Commutation methods applied to the mKdV. equation, Trans. Amer. Math. Soc. 324 (1991), 465-525.

[23] F. Geszlcsy and B. Simon, Uniqueness theorems in inverse spectral theory for one-dimensional Schrödinger operators, Trans. Amer. Math. Soc. 348 (1996), 349-373.

[24] F. Gesztesy and B. Simon, The xi function, Acta Math. 176 (1996), 49-71.

[25] F. Gesztesy, B. Simon and G. Teschl, Zeros of the Wronskian and renormalized oxcillation theory, Amer. J. Math. 118 (1996), 571-594.

[26] F. Gesztesy and R. Svirsky ( $m) K d V$-solitons on the background of quasi-periodic finite-gap solutions, Mem. Amer. Math. Soc. 118 (1995), no. 563.

[27] F. Gesztesy and G. Teschl, On the double commutation method, Proc. Amer. Math. Soc. 124 (1996), $1831-1840$.

[28] F. Gesztesy and G. Teschl, Commutation methods for Jacobi operators, J. Differential Equations 128 (1996), 252-299.

[29] F. Gesztesy and R. Weikard, Spectral deformations and soliton equations, in Differential Equations with Applications in Mathematical Physics (W. F. Amcs, E. M. Harrell and J. V. Herod, eds.), Academic Press, Boston, 1993, pp. 101-139.

[30] F. Gesztesy and Z. Zhao, On critical and subcritical Sturm-Liouville operators, J. Funct. Anal. 98 (1991), 311-345.

[31] H. Grosse and A. Martin, Particle Physics and the Schrödinger Equation, Cambridge University Press, Cambridge, to appear.

[32] K. Iwasaki, Inverse problem for Sturm-Liouville and Hill's equations, Ann. Mat. Pura Appl. (4) 149 (1987), 185-206.

[33] C. G. J. Jacobi, Zur Theorie der Variationsrechnung und der Differentialgleichungen, J. Reine Angew. Math. 17 (1837), 68-82.

[34] J. Kay and H. E. Moses, Reflectionless transmission through dielectrics and scattering potentials, J. Appl. Phys. 27 (1956), 1503-1508.

[35] E. A. Kuznetsov and A. V. Mikhailov, Stability of stationary waves in nonlinear weakly dispersive media, Soviet Phys. JETP 40 (1975), 855-859.

[36] W. Leighton, On self-adjoint differential equations of second order, J. London Math. Soc. 27 (1952), 37-47.

[37] B. M. Levitan, Inverse Sturm-Liouville Problems, VNU Science Press, Utrecht, 1987.

[38] B. M. Levitan, Sturm-Liouville operators on the whole line, with the same discrete spectrum, Math. USSR Sbornik 60 (1988), 77-106.

[39] V. A. Marchenko, Sturm-Liouville Operators and Applications, Birkhäuser, Basel, 1986.

[40] H. P. McKean, Variation on a theme of Jacobi, Comm. Pure Appl. Math. 38 (1985), 669-678.

[41] H. P. McKean, Geometry of $K d V(1)$ : Addition and the unimodular spectral classes, Rev. Mat. Iberoamericana 2 (1986), 235-261.

[42] H. P. McKean, Geometry of KdV (2): Three examples, J. Statist. Phys. 46 (1987), 1115-1143.

[43] H. P. McKean, Is there an infinite-dimensional algebraic geometry? Hints from $K d V$, in Theta Functions (L. Ehrenpreis and R.C. Gunning, eds.), Proc. Symp. Pure Math., Vol. 49, Amer. Math. Soc., Providence, RI, 1989, pp. 27-37.

[44] H. P. McKean, Geometry of KdV (3): Determinants and unimodular isospectral flows, Comm. Pure Appl. Math. 45 (1992), 389-415.

[45] H. P. McKean and E. Trubowitz, The spectral class of the quantum-mechanical harmonic oscillator, Comm. Math. Phys. 82 (1982), 471-495.

[46] H. P. McKean and P. van Moerbeke, The spectrum of Hill's equation, Invent. Math. 30 (1975), $217-274$

[47] S. Novikov, S. V. Manakov, L. P. Pitaevskii and V. E. Zakharov, Theory of Solitons, Consultants Bureau, New York, 1984.

[48] J. Pöschel and E. Trubowitz, Inverse Spectral Theory, Academic Press, Boston, 1987.

[49] J. Ralston and E. Trubowitz, Isospectral sets for boundary value problems on the unit interval, Ergodic Theory \& Dynamical Systems 8 (1988), 301-358.

[50] U.-W. Schmincke, On Schrödinger's factorization method for Sturm-Liouville operators, Proc. Roy. Soc. Edinburgh Sect. A 80 (1978), 67-84.

[51] B. Simon, Spectral analysis of rank one perturbations and applications, in CRM Proc. Lecture Notes, Vol. 8 (J. Feldman, R. Froese and L. Rosen, eds.), Amer. Math. Soc., Providence, RI, 1995, pp. 
$109-149$.

[52] G. Teschl, Oscillation and renormalized oscillation theory for Jucobi operators, to appear in J. Differential Equations.

[53] G. Teschl, Spectral deformations of Jacobi operators, preprint, 1996.

\author{
F. Gesztesy \\ DEPARTMENT OF MATHEMATICS \\ UNIVERSITY OF MISSOURI \\ COLUMBIA, MO 65211, USA \\ E-MAIL: FRITZ@MATH.MISSOURI.EDU \\ B. Simon \\ Division OF Physics, Mathematics AND Astronomy \\ CALIFORNIA INSTITUTE OF TECHNOLOGY \\ PASADENA, CA 91125, USA \\ E-MAIL: BSIMON@CALTECH.EDU \\ G. Teschl \\ INSTITUT FÜR REINE UND ANGEWANDTE MATHEMATIK \\ RWTH AACHEN \\ 52056 AACHEN, GERMANY \\ E-MAIL: GERALD@IRAM.RWTH-AACHEN.DE
}

(Received July 15, 1996) 